\title{
Orbits of real forms in complex flag manifolds
}

\author{
Andrea Altomani, Costantino Medori and Mauro Nacinovich
}

\begin{abstract}
We investigate the $C R$ geometry of the orbits $M$ of a real form $\mathbf{G}_{0}$ of a complex semisimple Lie group $\mathbf{G}$ in a complex flag manifold $X=\mathbf{G} / \mathbf{Q}$. We are mainly concerned with finite type and holomorphic nondegeneracy conditions, canonical $\mathbf{G}_{0}$-equivariant and Mostow fibrations, and topological properties of the orbits.
\end{abstract}

Mathematics Subject Classification (2010): 53C30 (primary); 14M15, 17B20, 32V05, 32V35, 32V40, 57T20 (secondary).

\section{Introduction}

In this paper we study a large class of homogeneous $C R$ manifolds, that come up as orbits of real forms in complex flag manifolds. These objects, that we call here parabolic CR manifolds, were first considered by J. A. Wolf (see [28] and also [12] for a comprehensive introduction to this topic). He studied the action of a real form $\mathbf{G}_{0}$ of a complex semisimple Lie group $\mathbf{G}$ on a flag manifold $X$ of $\mathbf{G}$. He showed that $\mathbf{G}_{0}$ has finitely many orbits in $X$, so that the union of the open orbits is dense in $X$, and that there is just one that is closed. He systematically investigated their properties, especially for the open orbits and for the holomorphic arc components of general orbits, outlining a framework that includes, as special cases, the bounded symmetric domains.

We aim here to give a contribution to the study of the general $\mathbf{G}_{0}$-orbits in $X$, by considering and utilizing their natural $C R$ structure.

In [2] we began this program by investigating the closed orbits. These can be combinatorially described in terms of their cross-marked Satake diagrams. This approach was possible because their isotropy subgroup always contains a maximally noncompact Cartan subgroup of $\mathbf{G}_{0}$. This is no longer the case for general orbits, and therefore we need here to deal with general Cartan subgroups. This different situation lead us to introduce the notions of adapted Cartan pairs and fit Weyl chambers, that we utilized to extend to general $\mathbf{G}_{0}$-orbits, and even simplify, the criteria of [2], that characterize finite type (see [7]) and holomorphic nondegeneracy (see [5]).

Received October 21, 2008; accepted in revised form March 19, 2009. 
A deeper analysis of the equivariant $\mathbf{G}_{0}$-maps between $\mathbf{G}_{0}$-orbits of various complex flag manifolds of $\mathbf{G}$ yields an accurate description of the smooth structure of the orbits. We show here (Theorem 7.2) that each orbit $M$ is $\mathbf{G}_{0}$-equivariantly equivalent to a tower of fibrations over a canonically associated real flag manifold $M_{\mathfrak{e}}$, with fibers that are products of Euclidean complex spaces and open orbits in complex flag manifolds. This result can be utilized to investigate some topological properties of $M$. It turns out, for instance, that the fundamental group $\pi_{1}(M)$ of $M$ only depends on $M_{\mathfrak{e}}$, and on the conjugacy class of the maximally noncompact Cartan subgroups of the isotropy of the action of $\mathbf{G}_{0}$ on $M$ (see Theorem 9.1). This explains why the fundamental group of a closed orbit $M$ is always isomorphic to that of $M_{\mathfrak{e}}$ (see e.g. [2, Section 8]).

Let $M$ be any $\mathbf{G}_{0}$-orbit in $X$. Fix a maximal compact subgroup $\mathbf{K}_{0}$ of $\mathbf{G}_{0}$, containing a maximal compact subgroup of the isotropy subgroup $\mathbf{I}_{0}$ of $M$, and let $\mathbf{K} \subset \mathbf{G}$ be its complexification. The Matsuki-dual (see e.g. $[9,17]$ ) complex Korbit $M^{*}$ intersects $M$ into a $\mathbf{K}_{0}$-orbit $N$, that is the basis of a Mostow fibration of $M$ (see [22,23]). We have $N=M$ when $M$ is closed, but otherwise $N$ is a $\mathbf{K}_{0}$-homogeneous $C R$ manifold with the same $C R$-codimension of $M$, but with a smaller $C R$-dimension. From the structure Theorem 7.2 we obtain that $N$ is $\mathbf{K}_{0^{-}}$ equivariantly a tower of fiber bundles, with basis $M_{\mathfrak{e}}$, and fibers that are complex flag manifolds. Since $N$ is a deformation retract of $M$, the $\mathbf{K}_{0}$-equivariant fibration $N \rightarrow M_{\mathfrak{e}}$ yields information on the topology of $M$ in terms of that of real and complex flag manifolds. The latter has been investigated by several Authors, see e.g. $[6,10,13,24,25]$.

We stress the fact that our methods and results are effective and permit to explicitly describe the objects involved and actually compute some of their invariants.

Let us turn now to a more detailed description of the contents of this paper. In the first section we collect some general definitions and results on $C R$ manifolds $M$, homogeneous for the transitive action of a real Lie group $\mathbf{G}_{0}$ of $C R$ automorphisms, and on their corresponding CR-algebras. These objects were defined in [21]: they are pairs $\left(\mathfrak{g}_{0}, \mathfrak{q}\right)$, where $\mathfrak{g}_{0}$ is the real Lie algebra of $\mathbf{G}_{0}$, and $\mathfrak{q}$ a complex Lie subalgebra of its complexification $\mathfrak{g}=\mathfrak{g}_{0}^{\mathbb{C}}$. Having fixed a point $x_{0} \in M$, the elements of $\mathfrak{q}$ correspond to the left invariant elements of the lift to $\mathbf{G}_{0}$, by the principal fibering $\mathbf{G}_{0} \ni g \rightarrow g \cdot x_{0} \in M$, of the complex tangent vector fields of type $(0,1)$ on $M$.

The notions of finite type and holomorphic degeneracy of $C R$ geometry translate, in the case of homogeneous $C R$ manifolds, into fundamentality and weak degeneracy of their $C R$ algebras (see Definition 1.5 and Proposition 1.6). When $M$ is not of finite type, or is holomorphically degenerate, then it was shown in [21] that there are nontrivial canonical $\mathbf{G}_{0}$-equivariant $C R$-fibrations $M \rightarrow M^{\prime}$, called the fundamental and the weakly nondegenerate reductions, respectively.

We need to make a remark to fill a gap between the results of [21] and the statement of Proposition 1.6 of this article. G. Fels (see [11]) pointed out to us that the notion of holomorphic degeneracy of [5], in the case of homogeneous $C R$ manifolds, coincides with our notion of weak degeneracy. This equivalence is used in point (3) of Proposition 1.6. 
In this paper, since we consider orbits of real forms in complex flag manifolds, we shall mostly restrict to $C R$ algebras $\left(\mathfrak{g}_{0}, \mathfrak{q}\right)$ that consist of a real form $\mathfrak{g}_{0}$ of a complex semisimple Lie algebra $\mathfrak{g}$ and of a complex parabolic Lie subalgebra $\mathfrak{q}$ of $\mathfrak{g}$. These were called parabolic $C R$ algebras in [2,21].

In Section 2 we collect some facts about complex flag manifolds, for which we refer to $[16,28]$. We describe the Chevalley decomposition of a complex parabolic subgroup $\mathbf{Q}$ of a semisimple complex linear group $\mathbf{G}$ (Proposition 2.3), to prepare for the description of the isotropy subgroup $\mathbf{I}_{0}$ of a $\mathbf{G}_{0}$-orbit $M$ in Section 3. There we obtain a suitable Chevalley decomposition of $\mathbf{I}_{0}$ (Proposition 3.4) from that of its Lie algebra $\mathfrak{i}_{0}$ (Proposition 3.2). Note that, in general, $\mathbf{I}_{0}$ is not a parabolic subgroup of $\mathbf{G}_{0}$, while this is true in the standard case, corresponding to the semisimple LeviTanaka algebras (see e.g. $[18,19]$ ). This Chevalley decomposition is fundamental to understanding the structure of the orbits. Our proof strongly relies on the semialgebraic nature of the objects under consideration, and on the properties of complex parabolic subgroups. In Section 3 we also consider the Cartan decomposition of the reductive factor of the Chevalley decomposition of $\mathbf{I}_{0}$, from which we derive information about the group of connected components of $\mathbf{I}_{0}$, in terms of its maximally noncompact Cartan subgroups (Theorem 3.6).

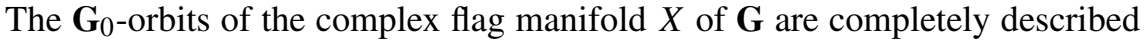
by their associated $C R$ algebras $\left(\mathfrak{g}_{0}, \mathfrak{q}\right)$. Recall that a complex parabolic subalgebra $\mathfrak{q}$ of $\mathfrak{g}$ contains a Borel subalgebra $\mathfrak{b}$ of $\mathfrak{g}$, and hence a Cartan subalgebra $\mathfrak{h}$ of $\mathfrak{g}$. Then $\mathfrak{q}$ is conveniently characterized in terms of the root system $\mathcal{R}$ defined by $\mathfrak{h}$. In fact, $\mathfrak{b}$ corresponds to a Weyl chamber $C$ of $\mathcal{R}$, and the parabolic $\mathfrak{q}$ 's containing $\mathfrak{b}$ are in one-to-one correspondence with the subsets $\Phi$ of the basis $\mathcal{B}$ of $C$-positive simple roots (see Formula (2.8)). Next, we observe that, for every real form $\mathfrak{g}_{0}$ of $\mathfrak{g}$, a complex parabolic subalgebra $\mathfrak{q}$ of $\mathfrak{g}$ always contains a Cartan subalgebra $\mathfrak{h}_{0}$ of $\mathfrak{g}_{0}$. It is convenient to employ the root system $\mathcal{R}$ of $\mathfrak{g}$ defined by the complexification $\mathfrak{h}$ of $\mathfrak{h}_{0}$, and Weyl chambers $C$ of $\mathcal{R}$ yielding a $\mathfrak{b} \subset \mathfrak{q}$. They are called fit for $\left(\mathfrak{g}_{0}, \mathfrak{q}\right)$ (Definition 2.1). Then the conjugation rules for the set $\mathcal{B}$ of simple $C$-positive roots, and the datum of the $\Phi \subset \mathcal{B}$ corresponding to $\mathfrak{q}$, encode all relevant information for the orbit $M$ associated to $\left(\mathfrak{g}_{0}, \mathfrak{q}\right)$.

In [2] it was natural to restrict to maximally noncompact Cartan subalgebras $\mathfrak{h}_{0}$ of $\mathfrak{g}_{0}$ and to fit Weyl chambers $C$ yielding Satake diagrams of $\mathfrak{g}_{0}$ (see [4]). Here the consideration of general orbits leads us first to consider all Cartan subalgebras $\mathfrak{h}_{0}$ of $\mathfrak{g}_{0}$, and then, after having fixed $\mathfrak{h}_{0}$, to select, among the fit Weyl chambers $C$, those for which $\mathcal{B}$ enjoys the best conjugation properties (see Lemma 4.3). These are called $S$-fit or $V$-fit, as they yield conjugation rules for $\mathcal{B}$ that are as close as possible to those of a Satake or a Vogan diagram, respectively (see Definition 4.4).

An important feature of parabolic $C R$ manifolds is the fact that to every $\mathfrak{g}_{0^{-}}$ equivariant morphism of parabolic $C R$ algebras (see [21]) always corresponds a smooth $\mathbf{G}_{0}$-equivariant fibration of the associated $\mathbf{G}_{0}$-orbits. We prove indeed that the inclusions between the isotropy subgroups of the $\mathbf{G}_{0}$-orbits are equivalent to those between their corresponding Lie subalgebras (Theorem 5.1). Besides providing the desired fibrations, this result also allows to consider changes of CR structures. This amounts to considering the given orbit $M$ simply as a homogeneous 
$\mathbf{G}_{0}$-manifold, and looking for its possible realizations as a real submanifold of a complex flag manifold of $\mathbf{G}$. In particular, we can inquire about maximal and minimal $\mathbf{G}_{0}$-homogeneous parabolic $C R$ structures on $M$. In fact, one of these, the weakening of the $C R$ structure (see Definition 5.4) will be one of the main ingredients in the proof of the structure Theorem 7.2. These $\mathbf{G}_{0}$-equivariant fibrations are not, in general, $C R$ maps. When they are $C R$, they are restrictions of $\mathbf{G}$-equivariant fibrations of flag manifolds, and their fibers $F$ have special features. Indeed, $F$ consists of finitely many connected components, each being a copy of a Cartesian product $F^{\prime} \times F^{\prime \prime}$, in which $F^{\prime}$ is the orbit of a real form in a suitable complex flag manifold $Y$, and $F^{\prime \prime}$ a complex nilmanifold (Theorem 5.10).

The $S$-fit Weyl chambers are especially suited to discuss finite type (see Theorem 6.2), while the $V$-fit Weyl chambers are the proper setting for weak degeneracy (see Proposition 6.3 and Theorem 6.4). Thus the results of Section 6 give a combinatoric way of constructing, in the special case of the orbits of a real form in a complex flag manifold, the basis of the fundamental and weakly nondegenerate reductions of a $C R$ algebra, that where discussed in general in [21, Section 5], and for the minimal orbits in [2, Sections 9,10,11]. The basis of these reductions can indeed be obtained by first representing the parabolic $\mathfrak{q}$ that characterizes $M$ by a subset $\Phi$ of $C$-positive simple roots for an $S$ - or $V$-fit Weyl chamber $C$, and then obtaining a new set $\Psi \subset \Phi$ by dropping roots of $\Phi$ according to some fairly easy rules, that are stated in Theorems 6.2, 6.4, respectively. These $\Psi$ give then the parabolic $\mathfrak{q}^{\prime}$ for which the $\left(\mathfrak{g}_{0}, \mathfrak{q}^{\prime}\right)$ are the basis of the desired reductions.

The construction of the fibration in the structure Theorem 7.2 is obtained by alternating weakenings of the $C R$ structures, that produce weak degeneracy, and the corresponding canonical weakly nondegenerate reductions, that have complex fibers (see Theorem 6.4). This process ends by yielding a fibering over a real flag manifold $M_{\mathfrak{e}}$. An important feature is that, although the fibering $M \rightarrow M_{\mathfrak{e}}$ may not be $C R$, at each step the weakly nondegenerate reductions are $C R$ fibration, and therefore we obtain a better control of the fibers (by Theorem 5.10). Our approach is a substitute for the use of holomorphic arc components in [28, Theorem 8.15].

If $M^{\prime} \rightarrow M$ is a $\mathbf{G}_{0}$-equivariant $C R$ fibering, with typical fiber $F$, the number of connected components of $F$ is proved to be equal to the quotient of the orders of the analytic Weyl groups of the reductive and of the semisimple part of the isotropy $\mathbf{I}_{0}$ of the basis, computed with respect to a maximally noncompact Cartan subgroup $\mathbf{H}_{0}$ of the reductive part of the isotropy $\mathbf{I}_{0}^{\prime}$ of the total space $M^{\prime}$ (Theorem 8.4).

The results of Section 7 and Section 8 are used, within Section 9 and the following Section 10, to study various topological properties of the $\mathbf{G}_{0}$-orbits $M$ and of the basis $N$ of their Mostow fibrations. In particular, we compute the first homotopy and homology groups of $M$ and $N$ (Theorem 9.1, Corollary 9.4 and Theorem 9.7). In Section 10 we show that the basis $N$ of the Mostow fibration $M \rightarrow N$ is a $C R$ manifold with the same $C R$ codimension of $M$ (Proposition 10.2), and then construct a $\mathbf{K}_{0}$-equivariant map $N \rightarrow M_{\mathfrak{e}}$ from $N$ to the real flag manifold $M_{\mathfrak{e}}$ (the real core of $M$ of Section 7), as a tower of fibrations, with fibers that are diffeomorphic to complex flag manifolds (Theorem 10.3). This allows, in Corollary 10.4, to re- 
late the Euler-Poincaré characteristics of $X, M_{\mathfrak{e}}$ and $N$ (see [3] for related results concerning the minimal orbits).

In the final section, Section 11, we compare our construction of the real core $M_{\mathfrak{e}}$ of $M$ with the space $M_{a}$ of its algebraic arc components, introduced in [28]. In many cases, e.g. when $M$ is a closed orbit, we have $M_{a}=M_{\mathfrak{e}}$, but it may happen for some $M$ that $M_{\mathfrak{e}}$ and $M_{a}$ are not diffeomorphic. We point out that the $\mathbf{G}_{0}$-equivariant fibration $M \rightarrow M_{\mathfrak{e}}$ has always simply connected fibers, while the fibers of $M \rightarrow M_{a}$ may not be simply connected.

\section{Homogeneous $C R$ manifolds and $C R$ algebras}

Let $M$ be a smooth manifold. A $C R$ structure on $M$ is the datum of a smooth complex subbundle $T^{0,1} M$ of its complexified tangent bundle $T^{\mathbb{C}} M$, with $T^{0,1} M \cap$ $\overline{T^{0,1} M}=0$, that is formally integrable, i.e. satisfies:

$$
\left[\Gamma\left(M, T^{0,1} M\right), \Gamma\left(M, T^{0,1} M\right)\right] \subset \Gamma\left(M, T^{0,1} M\right) .
$$

The complex rank $n$ of $T^{0,1} M$ is called the $C R$-dimension, and $k=\operatorname{dim}_{\mathbb{R}} M-2 n$ the $C R$-codimension of $M$. If $n=0$, we say that $M$ is totally real; if $k=0, M$ is a complex manifold in view of the Newlander-Nirenberg theorem.

Let $M$ be a real submanifold of a complex manifold $X$. For $x \in M$ set $T_{x}^{0,1} M=T_{x}^{0,1} X \cap T_{x}^{\mathbb{C}} M$. When the dimension of $T_{x}^{0,1} M$ is independent of $x \in M$, then $T^{0,1} M=\bigcup_{x \in M} T_{x}^{0,1} M$ is a formally integrable complex subbundle of $T^{\mathbb{C}} M$, defining on $M$ the structure of a $C R$ submanifold of $X$. If the complex dimension of $X$ is the sum of the $C R$ dimension and the $C R$ codimension of $M$, we say that the inclusion $M \hookrightarrow X$ is generic.

If $M$ and $M^{\prime}$ are $C R$ manifolds, a smooth map $f: M^{\prime} \rightarrow M$ is $C R$ if $d f^{\mathbb{C}}\left(T^{0,1} M^{\prime}\right) \subset T^{0,1} M$. In an obvious way, we define the notions of $C R$ immersion, submersion and diffeomorphism. In particular a smooth bundle $\pi: M^{\prime} \rightarrow M$ for which $\pi$ is a $C R$ submersion (i.e. $\pi$ is a $C R$ map and $d \pi^{\mathbb{C}}\left(T^{0,1} M^{\prime}\right)=T^{0,1} M$ ) is called a $C R$ bundle or a $C R$ fibration.

Definition 1.1. Let $\mathbf{G}_{0}$ be a Lie group. A $\mathbf{G}_{0}$-homogeneous $C R$ manifold is a $\mathbf{G}_{0^{-}}$ homogeneous smooth manifold endowed with a $\mathbf{G}_{0}$-invariant $C R$ structure.

Fix a point $x_{0}$ of a $\mathbf{G}_{0}$-homogeneous $C R$ manifold $M$, let $\mathbf{I}_{0} \subset \mathbf{G}_{0}$ be the isotropy subgroup of $x_{0}$ and $\pi: \mathbf{G}_{0} \rightarrow M$ the corresponding principal $\mathbf{I}_{0}$-bundle. Denote by $\mathfrak{Z}\left(\mathbf{G}_{0}\right)$ the space of smooth sections of the pullback by $\pi$ of $T^{0,1} M$ to $\mathbf{G}_{0}$, i.e. the set of complex valued vector fields $Z$ in $\mathbf{G}_{0}$ such that $d \pi^{\mathbb{C}}\left(Z_{g}\right) \in T_{\pi(g)}^{0,1} M$ for all $g \in \mathbf{G}_{0}$. By (1.1), also $\mathfrak{Z}\left(\mathbf{G}_{0}\right)$ is formally integrable, i.e. $\left[\mathfrak{Z}\left(\mathbf{G}_{0}\right), \mathfrak{Z}\left(\mathbf{G}_{0}\right)\right] \subset$ $\mathfrak{Z}\left(\mathbf{G}_{0}\right)$. Moreover, $\mathfrak{Z}\left(\mathbf{G}_{0}\right)$ is invariant by left translations in $\mathbf{G}_{0}$. Hence its left invariant vector fields generate $\mathfrak{Z}\left(\mathbf{G}_{0}\right)$ as a left $\mathcal{C}^{\infty}\left(\mathbf{G}_{0}, \mathbb{C}\right)$-module. 
Thus, denoting by $\mathfrak{g}$ the complexification of the Lie algebra $\mathfrak{g}_{0}$ of $\mathbf{G}_{0}$, by the formal integrability condition (1.1), the subspace

$$
\mathfrak{q}=\left(d \pi^{\mathbb{C}}\right)^{-1}\left(T_{x_{0}}^{0,1} M\right) \subset \mathfrak{g}=T_{e}^{\mathbb{C}} \mathbf{G}_{0}
$$

is a complex subalgebra of $\mathfrak{g}$, and is $\operatorname{Ad}_{\mathfrak{g}}\left(\mathbf{I}_{0}\right)$-invariant. We can summarize these observations by:

Lemma 1.2. Let $\mathfrak{i}_{0}$ be the Lie algebra of the isotropy $\mathbf{I}_{0}$. Then (1.2) establishes a one-to-one correspondence between the $\mathbf{G}_{0}$-homogeneous $C R$ structures on $M=$ $\mathbf{G}_{0} / \mathbf{I}_{0}$ and the $\operatorname{Ad}_{\mathfrak{g}}\left(\mathbf{I}_{0}\right)$-invariant complex Lie subalgebras $\mathfrak{q}$ of $\mathfrak{g}$ such that $\mathfrak{q} \cap \mathfrak{g}_{0}=\mathfrak{i}_{0}$.

This was our motivation to introduce and discuss CR algebras in [21]. We rehearse some definitions.

Definition 1.3. A $C R$ algebra is a pair $\left(\mathfrak{g}_{0}, \mathfrak{q}\right)$, consisting of a real Lie algebra $\mathfrak{g}_{0}$ and of a complex Lie subalgebra $\mathfrak{q}$ of its complexification $\mathfrak{g}$, such that the quotient $\mathfrak{g}_{0} /\left(\mathfrak{q} \cap \mathfrak{g}_{0}\right)$ is a finite dimensional real vector space.

If $M$ is a $\mathbf{G}_{0}$-homogeneous $C R$ manifold and $\mathfrak{q}$ is defined by (1.2), we say that the $C R$ algebra $\left(\mathfrak{g}_{0}, \mathfrak{q}\right)$ is associated to $M$.

Remark 1.4. The $C R$-dimension and $C R$-codimension of $M$ can be computed in terms of its associated $C R$ algebra $\left(\mathfrak{g}_{0}, \mathfrak{q}\right)$. We have indeed

$$
\begin{aligned}
C R-\operatorname{dim} M & =\operatorname{dim}_{\mathbb{C}} \mathfrak{q}-\operatorname{dim}_{\mathbb{C}}(\mathfrak{q} \cap \overline{\mathfrak{q}}), \\
C R-\operatorname{codim} M & =\operatorname{dim}_{\mathbb{C}} \mathfrak{g}-\operatorname{dim}_{\mathbb{C}}(\mathfrak{q}+\overline{\mathfrak{q}}) .
\end{aligned}
$$

The $C R$ algebra $\left(\mathfrak{g}_{0}, \mathfrak{q}\right)$ is said to be totally real when $C R$-dim $M=0$, totally complex when $C R$-codim $M=0$.

Definition 1.5. A $C R$ algebra $\left(\mathfrak{g}_{0}, \mathfrak{q}\right)$ is called:

- fundamental if there is no complex Lie subalgebra $\mathfrak{q}^{\prime}$ of $\mathfrak{g}$ with:

$$
\mathfrak{q}+\overline{\mathfrak{q}} \subset \mathfrak{q}^{\prime} \subsetneq \mathfrak{g}
$$

- strictly, or Levi-nondegenerate if

$$
\{Z \in \mathfrak{q} \mid \operatorname{ad}(Z)(\overline{\mathfrak{q}}) \subset \mathfrak{q}+\overline{\mathfrak{q}}\}=\mathfrak{q} \cap \overline{\mathfrak{q}}
$$

- weakly degenerate if there is a complex Lie subalgebra $\mathfrak{q}^{\prime}$ of $\mathfrak{g}$ with:

$$
\mathfrak{q} \varsubsetneqq \mathfrak{q}^{\prime} \subset \mathfrak{q}+\overline{\mathfrak{q}} .
$$


We have:

Proposition 1.6. Let $M$ be a $\mathbf{G}_{0}$-homogeneous $C R$ manifold with associated $C R$ algebra $\left(\mathfrak{g}_{0}, \mathfrak{q}\right)$. Then:

(1) $\left(\mathfrak{g}_{0}, \mathfrak{q}\right)$ is fundamental if and only if $M$ is of finite type in the sense of [7].

(2) $\left(\mathfrak{g}_{0}, \mathfrak{q}\right)$ is strictly nondegenerate if and only if the vector valued Levi form (see e.g. [20]) on M is nondegenerate. Strict nondegeneracy implies weak nondegeneracy.

(3) $\left(\mathfrak{g}_{0}, \mathfrak{q}\right)$ is weakly nondegenerate and fundamental if and only if the group of germs of CR diffeomorphisms at $x_{0} \in M$ that stabilize $x_{0}$ is a finite dimensional Lie group, i.e. $M$ is holomorphically nondegenerate (see e.g. [5,11]).

(4) $\left(\mathfrak{g}_{0}, \mathfrak{q}\right)$ is weakly degenerate if and only if there exists a local $\mathbf{G}_{0}$-equivariant $C R$ fibration $M \rightarrow M^{\prime}$, with nontrivial complex fibers.

Proof. All the statements, except (3), were proved in [21, Proposition 13.3] and [2, Proposition 4.1]. Item (3) follows from the observation in [11] that, for homogeneous $C R$ manifolds, weak nondegeneracy is equivalent to the finite nondegeneracy of [11], which is in turn equivalent to the holomorphic nondegeneracy of [5].

\section{Complex flag manifolds}

In this section we collect some notions on complex flag manifolds.

A complex flag manifold is the quotient $X=\mathbf{G} / \mathbf{Q}$ of a connected complex semisimple Lie group $\mathbf{G}$ by a parabolic subgroup $\mathbf{Q}$. We recall that $\mathbf{Q}$ is parabolic in $\mathbf{G}$ if and only if its Lie algebra $\mathfrak{q}$ is a parabolic Lie subalgebra of the Lie algebra $\mathfrak{g}$ of $\mathbf{G}$. This means that $\mathfrak{q}$ contains a Borel subalgebra, i.e. a maximal solvable Lie subalgebra of $\mathfrak{g}$. We also note that $\mathbf{G}$ is necessarily a linear group, and that $\mathbf{Q}$ is connected, contains the center of $\mathbf{G}$ and equals the normalizer of $\mathfrak{q}$ in $\mathbf{G}$ :

$$
\mathbf{Q}=\{g \in \mathbf{G} \mid \operatorname{Ad}(g)(\mathfrak{q})=\mathfrak{q}\}
$$

The isotropy subgroup of a point $x=g \mathbf{Q}$ of $X$ is $\operatorname{ad}(g)(\mathbf{Q})$. Since $\mathbf{Q}$ is its own normalizer in $\mathbf{G}$, we can identify $x$ with $\operatorname{ad}(g)(\mathbf{Q})$. Moreover, since $\mathbf{Q}$ is connected, $\operatorname{ad}(g)(\mathbf{Q})$ is completely determined by its Lie algebra $\operatorname{Ad}(g)(\mathfrak{q})$. Thus $X$ can be viewed as the space of parabolic subalgebras of $\mathfrak{g}$ that are $\operatorname{Ad}(\mathbf{G})$-conjugate to $\mathfrak{q}$. Hence we obtain an isomorphic $X$ by substituting $\operatorname{Int}_{\mathbb{C}}(\mathfrak{g})$ to $\mathbf{G}$.

In particular, a different choice of a connected $\mathbf{G}^{\prime}$ and of a parabolic $\mathbf{Q}^{\prime}$, with Lie algebras $\mathfrak{g}^{\prime}$ and $\mathfrak{q}^{\prime}$ isomorphic to $\mathfrak{g}$ and $\mathfrak{q}$, yields a complex flag manifold $X^{\prime}$ that is complex-projectively isomorphic to $X$. Thus a flag manifold $X$ is completely described by the pair consisting of the Lie algebras $\mathfrak{g}$ and $\mathfrak{q}$.

Fix a Cartan subalgebra $\mathfrak{h}$ of $\mathfrak{g}$, contained in $\mathfrak{q}$, and let $\mathcal{R}=\mathcal{R}(\mathfrak{g}, \mathfrak{h})$ be the corresponding root system. For each $\alpha \in \mathcal{R}$, let $\mathfrak{g}^{\alpha}=\{Z \in \mathfrak{g} \mid[H, Z]=$ $\alpha(H) Z, \forall H \in \mathfrak{h}\}$ be the root subspace of $\alpha$. Denote by $\mathfrak{h}_{\mathbb{R}}$ the real subspace of $\mathfrak{h}$ 
on which the roots are real valued. The choice of a Weyl chamber $C \subset \mathfrak{h}_{\mathbb{R}}$ defines a partial order $\prec$ in the dual space $\mathfrak{h}_{\mathbb{R}}^{*}$ of $\mathfrak{h}_{\mathbb{R}}$. Since $\mathfrak{q}$ contains $\mathfrak{h}$, it is the direct sum of $\mathfrak{h}$ and of the root spaces contained in $\mathfrak{q}$, i.e.

$$
\mathfrak{q}=\mathfrak{h}+\sum_{\alpha \in \mathcal{Q}} \mathfrak{g}^{\alpha}, \quad \text { where } \quad \mathcal{Q}=\left\{\alpha \in \mathcal{R} \mid \mathfrak{g}^{\alpha} \subset \mathfrak{q}\right\}
$$

Then the fact that $\mathfrak{q}$ is parabolic means that one can choose $C$ in such a way that:

$$
\alpha \in \mathcal{Q} \text { for all } \alpha \succ 0 \text {. }
$$

The set $\mathcal{Q}$ is parabolic, i.e. is closed under root addition and $\mathcal{Q} \cup(-\mathcal{Q})=\mathcal{R}$.

Definition 2.1. A Weyl chamber $C$ for which (2.3) holds true is called fit for $\mathcal{Q}$. We denote by $\mathfrak{C}(\mathcal{R})$ the set of all Weyl chambers for $\mathcal{R}$ and by $\mathfrak{C}(\mathcal{R}, \mathcal{Q})$ the subset of those that are fit for $\mathcal{Q}$.

Let $C \in \mathfrak{C}(\mathcal{R})$ and $\mathcal{B}=\mathcal{B}(C)$ be the corresponding system of $C$-positive simple roots. All $\alpha \in \mathcal{R}$ are linear combinations of elements of the basis $\mathcal{B}$ :

$$
\alpha=\sum_{\beta \in \mathcal{B}} k_{\alpha}^{\beta} \beta, \quad k_{\alpha}^{\beta} \in \mathbb{Z} .
$$

Definition 2.2. We define the support $\operatorname{supp}(\alpha)$ of $\alpha$ with respect to $\mathcal{B}$ as the set of $\beta \in \mathcal{B}$ for which $k_{\alpha}^{\beta} \neq 0$.

Having fixed $C \in \mathfrak{C}(\mathcal{R}, \mathcal{Q})$, we associate to $\mathfrak{q}$ the subset $\Phi$ of $\mathcal{B}$, consisting of the simple $C$-positive roots $\alpha$ for which $\mathfrak{g}^{-\alpha} \not \subset \mathfrak{q}$.

Then $\mathcal{Q}$ and $\mathfrak{q}$ are completely determined by $\Phi$. Indeed:

$$
\begin{aligned}
\mathcal{Q} & =\mathcal{Q}_{\Phi}=\{\alpha \succ 0\} \cup\{\alpha \prec 0 \mid \operatorname{supp}(\alpha) \cap \Phi=\emptyset\}=\mathcal{Q}_{\Phi}^{n} \cup \mathcal{Q}_{\Phi}^{r}, \text { with: } \\
\mathcal{Q}^{n} & =\mathcal{Q}_{\Phi}^{n}=\{\alpha \in \mathcal{R} \mid \alpha \succ 0 \text { and } \operatorname{supp}(\alpha) \cap \Phi \neq \emptyset\}, \\
\mathcal{Q}^{r} & =\mathcal{Q}_{\Phi}^{r}=\{\alpha \in \mathcal{R} \mid \operatorname{supp}(\alpha) \cap \Phi=\emptyset\}
\end{aligned}
$$

and for the parabolic subalgebra $\mathfrak{q}$ we have the decomposition:

$$
\begin{aligned}
& \mathfrak{q}=\mathfrak{q}_{\Phi}=\mathfrak{h}+\sum_{\alpha \in \mathcal{Q}_{\Phi}} \mathfrak{g}^{\alpha}=\mathfrak{q}^{r} \oplus \mathfrak{q}^{n}, \quad \text { where: } \\
& \mathfrak{q}^{n}=\mathfrak{q}_{\Phi}^{n}=\sum_{\alpha \in \mathcal{Q}_{\Phi}^{n}} \mathfrak{g}^{\alpha} \quad \text { is the nilradical of } \mathfrak{q} \text { and } \\
& \mathfrak{q}^{r}=\mathfrak{q}_{\Phi}^{r}=\mathfrak{h}+\sum_{\alpha \in \mathcal{Q}_{\Phi}^{r}} \mathfrak{g}^{\alpha} \text { is a reductive complement of } \mathfrak{q}_{\Phi}^{n} \text { in } \mathfrak{q}_{\Phi}
\end{aligned}
$$

We explicitly note that $\Phi$ is related to $\mathcal{Q}$ and $C$ by

$$
\Phi=\mathcal{Q}^{n} \cap \mathcal{B}
$$


All Cartan subalgebras $\mathfrak{h}$ of $\mathfrak{g}$ are equivalent, modulo inner automorphisms. After $\mathfrak{h}$, and hence $\mathcal{R}$, have been fixed, all basis of simple roots of $\mathcal{R}$ are equivalent for the transpose of inner automorphisms of $\mathfrak{g}$ normalizing $\mathfrak{h}$. Thus, after picking a Weyl chamber $C$, and having fixed in this way a system $\mathcal{B}$ of $C$-positive simple roots, the correspondence $\Phi \leftrightarrow \mathfrak{q}_{\Phi}$ is one-to-one between subsets $\Phi$ of $\mathcal{B}$ and complex parabolic Lie subalgebras of $\mathfrak{g}$, modulo inner automorphisms. In other words, the subsets $\Phi$ of our fixed $\mathcal{B}$ parametrize all different flag manifolds $X$ of a connected semisimple complex Lie group $\mathbf{G}$ with Lie algebra $\mathfrak{g}$.

The choice of a Cartan subalgebra $\mathfrak{h}$ of $\mathfrak{g}$ contained in $\mathfrak{q}$ yields a canonical Chevalley decomposition of the parabolic subgroup $\mathbf{Q}$ :

Proposition 2.3. Let $\mathbf{Q}$ be a parabolic subgroup of $\mathbf{G}$, corresponding to the complex parabolic Lie subalgebra $\mathfrak{q}$ of $\mathfrak{g}$. With the notation above, we have a Chevalley decomposition:

$$
\mathbf{Q}=\mathbf{Q}^{n} \rtimes \mathbf{Q}^{r}
$$

where the unipotent radical $\mathbf{Q}^{n}$ is the connected and simply connected Lie subgroup of $\mathbf{G}$ with Lie algebra $\mathfrak{q}^{n}$ given by (2.9), and $\mathbf{Q}^{r}$ is the reductive ${ }^{1}$ complement of $\mathbf{Q}^{n}$ in $\mathbf{Q}$, whose Lie algebra $\mathfrak{q}^{r}$ is given by (2.10).

Let $\mathfrak{c} \subset \mathfrak{h}$ be the center of $\mathfrak{q}^{r}$ :

$$
\mathfrak{c}=\mathfrak{z}\left(\mathfrak{q}^{r}\right)=\left\{H \in \mathfrak{h} \mid \operatorname{ad}(H)\left(\mathfrak{q}^{r}\right)=0\right\}
$$

Then the reductive complement $\mathbf{Q}^{r}$ is characterized by:

$$
\mathbf{Q}^{r}=\mathbf{Z}_{\mathbf{G}}(\mathfrak{c})=\{g \in \mathbf{G} \mid \operatorname{Ad}(g)(H)=H \quad \forall H \in \mathfrak{c}\}
$$

Moreover, $\mathbf{Q}^{r}$ is a subgroup of finite index in the normalizer $\mathbf{N}_{\mathbf{G}}\left(\mathfrak{q}^{r}\right)$ of $\mathfrak{q}^{r}$ in $\mathbf{G}$, and $\mathbf{Q} \cap \mathbf{N}_{\mathbf{G}}\left(\mathfrak{q}^{r}\right)=\mathbf{Q}^{r}$

Proof. A complex parabolic subgroup can also be considered as a real parabolic subgroup. The Chevalley decomposition (2.12) reduces then to the Langlands decomposition $\mathbf{Q}=\mathbf{M A N}$, with $\mathbf{N}=\mathbf{Q}^{n}$ and $\mathbf{M A}=\mathbf{Q}^{r}$. Thus our statement is a consequence of [16, Proposition 7.82(a)].

Note that $\mathfrak{q}^{r}$ is the centralizer of $\mathfrak{c}$ in $\mathfrak{g}$ and is its own normalizer. This yields the inclusion $\mathbf{Q}^{r} \subset \mathbf{N}_{\mathbf{G}}\left(\mathfrak{q}^{r}\right)$. Since $\mathbf{N}_{\mathbf{G}}\left(\mathfrak{q}^{r}\right)$ is semi-algebraic, it has finitely many connected components. Thus its intersection with $\mathbf{Q}^{n}$ is discrete and finite, and thus trivial because $\mathbf{Q}^{n}$ is connected, simply connected and unipotent.

1 According to [16] we call reductive a linear Lie group $\mathbf{G}_{0}$, having finitely many connected components, with a reductive Lie algebra $\mathfrak{g}_{0}$, and such that, for the complexification $\mathfrak{g}$ of $\mathfrak{g}_{0}$, we have $\operatorname{Ad}_{\mathfrak{g}}\left(\mathbf{G}_{0}\right) \subset \operatorname{Int}_{\mathbb{C}}(\mathfrak{g})$. While stating here the reductiveness of $\mathbf{Q}^{r}$, we consider the given group $\mathbf{G}$ as a real linear group. 


\section{3. $G_{0}$-orbits and their isotropy subgroups}

We keep the notation of Section 2. Let $X=\mathbf{G} / \mathbf{Q}$ be a complex flag manifold, and $\mathbf{G}_{0}$ a connected real form of $\mathbf{G}$. Note that $\mathbf{G}_{0}$ is semi-algebraic, being a connected component of an algebraic group for the Euclidean topology. We know from [28] that there are finitely many $\mathbf{G}_{0}$-orbits in $X$. Fix any such orbit $M$ and a point $x \in M$. We can assume that $\mathbf{Q} \subset \mathbf{G}$ is the stabilizer of $x$ for the action of $\mathbf{G}$ in $X$. Let $\mathbf{I}_{0}=\mathbf{Q} \cap \mathbf{G}_{0}$ be the stabilizer of $x$ in $\mathbf{G}_{0}$, so that $M \simeq \mathbf{G}_{0} / \mathbf{I}_{0}$, as a smooth manifold. The Lie algebra $\mathfrak{g}_{0}$ of $\mathbf{G}_{0}$ is a real form of $\mathfrak{g}$ and the Lie algebra of the isotropy subgroup $\mathbf{I}_{0}$ is the intersection $\mathfrak{i}_{0}=\mathfrak{q} \cap \mathfrak{g}_{0}$, where $\mathfrak{q}$ is the Lie algebra of $\mathbf{Q}$.

Since $\mathbf{I}_{0}$ always contains the center of $\mathbf{G}_{0}$, the orbit $M$ actually only depends on the pair of Lie subalgebras $\mathfrak{g}_{0}$ and $\mathfrak{q}$ of $\mathfrak{g}$.

Definition 3.1. A pair $\left(\mathfrak{g}_{0}, \mathfrak{q}\right)$, consisting of a semisimple real Lie algebra $\mathfrak{g}_{0}$ and of a complex parabolic Lie subalgebra $\mathfrak{q}$ of its complexification $\mathfrak{g}$ is a parabolic $C R$ algebra. The corresponding $\mathbf{G}_{0}$-orbit $M$ in $X=\mathbf{G} / \mathbf{Q}$ is said to be a parabolic $C R$ manifold.

We summarize the results of [2, page 491] by stating the following:

Proposition 3.2 (Decomposition of the isotropy subalgebra). Let $\left(\mathfrak{g}_{0}, \mathfrak{q}\right)$ be a parabolic CR algebra, and $\mathfrak{i}_{0}=\mathfrak{q} \cap \mathfrak{g}_{0}$ the corresponding isotropy subalgebra. Then $\mathfrak{i}_{0}$ contains a Cartan subalgebra $\mathfrak{h}_{0}$ of $\mathfrak{g}_{0}$.

If $\mathfrak{h}_{0}$ is any Cartan subalgebra of $\mathfrak{g}_{0}$ contained in $\mathfrak{i}_{0}$, there is a Cartan involution $\vartheta: \mathfrak{g}_{0} \rightarrow \mathfrak{g}_{0}$, with $\vartheta\left(\mathfrak{h}_{0}\right)=\mathfrak{h}_{0}$, yielding a decomposition

$$
\mathfrak{i}_{0}=\mathfrak{n}_{0} \oplus \mathfrak{l}_{0}=\mathfrak{n}_{0} \oplus \mathfrak{s}_{0} \oplus \mathfrak{z}_{0}
$$

such that:

(1) $\mathfrak{n}_{0}$ is the nilpotent ideal of $\mathfrak{i}_{0}$, consisting of the elements $Y \in \mathfrak{i}_{0}$ for which $\operatorname{ad}_{\mathfrak{g}_{0}}(Y)$ is nilpotent;

(2) $\mathfrak{l}_{0}=\mathfrak{s}_{0} \oplus \mathfrak{z}_{0}$ is reductive;

(3) $\mathfrak{z}_{0} \subset \mathfrak{h}_{0}$ is the center of $\mathfrak{l}_{0}$ and $\mathfrak{s}_{0}=\left[\mathfrak{l}_{0}, \mathfrak{l}_{0}\right]$ its semisimple ideal;

(4) $\mathfrak{n}_{0}=\left[\mathfrak{z}_{0}, \mathfrak{n}_{0}\right]=\left[\mathfrak{z}_{0}, \mathfrak{i}_{0}\right]$;

(5) $\mathfrak{l}_{0}=\mathfrak{i}_{0} \cap \vartheta\left(\mathfrak{i}_{0}\right)$ is a $\vartheta$-invariant Lie subalgebra of $\mathfrak{g}_{0}$, and also $\mathfrak{z}_{0}$ and $\mathfrak{s}_{0}$ are $\vartheta$-invariant;

(6) $\mathfrak{s}_{0}$ and $\mathfrak{z}_{0}$ are orthogonal for the Killing form of $\mathfrak{g}_{0}$;

(7) the complexifications $\mathfrak{n}$ of $\mathfrak{n}_{0}$ and $\mathfrak{l}$ of $\mathfrak{l}_{0}$ are:

$$
\mathfrak{n}=\mathfrak{q}^{n} \cap \overline{\mathfrak{q}}+\overline{\mathfrak{q}}^{n} \cap \mathfrak{q}, \quad \mathfrak{l}=\mathfrak{q}^{r} \cap \overline{\mathfrak{q}}^{r} .
$$

Definition 3.3. Let $\left(\mathfrak{g}_{0}, \mathfrak{q}\right)$ be a parabolic $C R$ algebra. A pair $\left(\vartheta, \mathfrak{h}_{0}\right)$, consisting of a Cartan involution $\vartheta$ of $\mathfrak{g}_{0}$ and of a $\vartheta$-invariant Cartan subalgebra $\mathfrak{h}_{0}$ of $\mathfrak{i}_{0}$ will be said to be a Cartan pair adapted to $\left(\mathfrak{g}_{0}, \mathfrak{q}\right)$. 
We have the following:

Proposition 3.4 (Chevalley decomposition of the isotropy). Keep the notation introduced above. The isotropy subgroup $\mathbf{I}_{0}$ is the closed real semi-algebraic subgroup of $\mathbf{G}_{0}$ :

$$
\mathbf{I}_{0}=\mathbf{N}_{\mathbf{G}_{0}}(\mathfrak{q})=\left\{g \in \mathbf{G}_{0} \mid \operatorname{Ad}_{\mathfrak{g}}(g)(\mathfrak{q})=\mathfrak{q}\right\} .
$$

The isotropy subgroup $\mathbf{I}_{0}$ admits a Chevalley decomposition

$$
\mathbf{I}_{0}=\mathbf{L}_{0} \ltimes \mathbf{N}_{0}
$$

where:

(1) $\mathbf{N}_{0}$ is a unipotent, closed, connected, and simply connected normal subgroup of $\mathbf{I}_{0}$, with Lie algebra $\mathfrak{n}_{0}$;

(2) $\mathbf{L}_{0}$ is a reductive Lie subgroup, with Lie algebra $\mathfrak{l}_{0}$, and is the centralizer of $\mathfrak{z}_{0}$ in $\mathbf{G}_{0}$ :

$$
\mathbf{L}_{0}=\mathbf{Z}_{\mathbf{G}_{0}}\left(\mathfrak{z}_{0}\right)=\left\{g \in \mathbf{G}_{0} \mid \operatorname{Ad}_{\mathfrak{g}_{0}}(g)(H)=H \quad \forall H \in \mathfrak{z}_{0}\right\} .
$$

Proof. Formula (3.3) holds because $\mathbf{Q}$ is the normalizer of $\mathfrak{q}$ in $\mathbf{G}$.

Fix a Cartan pair $\left(\vartheta, \mathfrak{h}_{0}\right)$ adapted to $\left(\mathfrak{g}_{0}, \mathfrak{q}\right)$. We consider the complexification $\mathfrak{h}$ of $\mathfrak{h}_{0}$ and use the notation of (2.5)-(2.10). Let us construct a parabolic $\mathbf{Q}^{\prime} \subset \mathbf{Q}$ whose reductive part $\mathbf{Q}^{\prime r}$ is a complexification of $\mathbf{L}_{0}$. To this aim we set

$$
\mathfrak{q}^{\prime}=\mathfrak{q}^{n} \oplus\left(\mathfrak{q}^{r} \cap \overline{\mathfrak{q}}\right)
$$

We claim that $\mathfrak{q}^{\prime}$ is a parabolic Lie subalgebra of $\mathfrak{g}$. It contains the Cartan subalgebra $\mathfrak{h}$, because $\mathfrak{h} \subset \mathfrak{q}^{r} \cap \overline{\mathfrak{q}}^{r}$. Since $\mathfrak{q}^{\prime}$ is clearly a complex Lie subalgebra of $\mathfrak{q}$, to show that $\mathfrak{q}^{\prime}$ is parabolic it suffices to verify that, for each $\alpha \in \mathcal{R}$ (the root system of $\mathfrak{g}$ with respect to $\mathfrak{h}$ ) either $\mathfrak{g}^{\alpha}$ or $\mathfrak{g}^{-\alpha}$ is contained in $\mathfrak{q}^{\prime}$. This is obvious if $\{\alpha,-\alpha\} \cap \mathcal{Q}^{n} \neq \emptyset$. We need only to consider roots $\alpha$ with $\pm \alpha \in \mathcal{Q}^{r}$, and then observe that either $\mathfrak{g}^{\alpha}$ or $\mathfrak{g}^{-\alpha}$ is contained in $\overline{\mathfrak{q}}$, because $\overline{\mathfrak{q}}$ is parabolic and contains $\mathfrak{h}$. Moreover, one easily verifies that

$$
\mathfrak{q}^{\prime r}=\overline{\mathfrak{q}}^{\prime r} \quad \text { and } \quad \mathfrak{q}^{\prime}=\mathfrak{q}^{n}+(\mathfrak{q} \cap \overline{\mathfrak{q}}) .
$$

Hence, the center $\mathfrak{c}^{\prime}$ of $\mathfrak{q}^{\prime r}$ coincides with the complexification $\mathfrak{z}$ of $\mathfrak{z}_{0}$.

If $g \in \mathbf{I}_{0}$, then $\operatorname{Ad}_{\mathfrak{g}_{0}}(g)\left(\mathfrak{l}_{0}\right)$ is a reductive complement of $\mathfrak{n}_{0}$ in $\mathfrak{i}_{0}$. Since all reductive complements of $\mathfrak{n}_{0}$ in $\mathfrak{i}_{0}$ are conjugated by an inner automorphism from $\operatorname{Ad}_{\mathfrak{i}_{0}}\left(\mathbf{N}_{0}\right)$, we can find a $g_{n} \in \mathbf{N}_{0}$ such that $\operatorname{Ad}_{\mathfrak{i}_{0}}\left(g_{n}^{-1} g\right)\left(\mathfrak{l}_{0}\right)=\mathfrak{l}_{0}$. Consider the element $g_{r}=g_{n}^{-1} g$. We have:

$$
\begin{array}{ll}
\operatorname{Ad}_{\mathfrak{g}_{0}}\left(g_{r}\right)\left(\mathfrak{g}_{0}\right)=\mathfrak{g}_{0}, & \operatorname{Ad}_{\mathfrak{g}}\left(g_{r}\right)(\mathfrak{q})=\mathfrak{q}, \\
\operatorname{Ad}_{\mathfrak{g}}\left(g_{r}\right)\left(\mathfrak{q}^{n}\right)=\mathfrak{q}^{n}, & \operatorname{Ad}_{\mathfrak{g}}\left(g_{r}\right)(\overline{\mathfrak{q}})=\overline{\mathfrak{q}},
\end{array}
$$

because $g_{r} \in \mathbf{Q} \cap \overline{\mathbf{Q}}$. Thus $\operatorname{Ad}_{\mathfrak{g}}\left(g_{r}\right)\left(\mathfrak{q}^{\prime}\right)=\mathfrak{q}^{\prime}$. Moreover, $\operatorname{Ad}_{\mathfrak{g}}\left(g_{r}\right)\left(\mathfrak{q}^{r}\right)=\mathfrak{q}^{r}$ and $\operatorname{Ad}_{\mathfrak{g}}\left(g_{r}\right)\left(\overline{\mathfrak{q}}^{r}\right)=\overline{\mathfrak{q}}^{r}$. Since $\mathfrak{q}^{\prime r}=\mathfrak{q}^{r} \cap \overline{\mathfrak{q}}^{r}$, we obtain that $\operatorname{Ad}_{\mathfrak{g}}\left(g_{r}\right)\left(\mathfrak{q}^{\prime r}\right)=\mathfrak{q}^{\prime r}$. Hence, by Proposition 2.3, $g_{r}$ belongs to $\mathbf{Q}^{\prime r} \cap \mathbf{I}_{0}$, and the statement follows. Note that in fact we obtain $\mathbf{L}_{0}=\mathbf{Q}^{\prime r} \cap \mathbf{G}_{0}$, so that (3.5) is a consequence of Proposition 2.3. 
Corollary 3.5. We keep the notation and the assumptions of Propositions 3.2 and 3.4. Let $\left(\vartheta, \mathfrak{h}_{0}\right)$ be a Cartan pair adapted to $\left(\mathfrak{g}_{0}, \mathfrak{q}\right)$ and

$$
\mathfrak{g}_{0}=\mathfrak{k}_{0} \oplus \mathfrak{p}_{0} \quad \text { and } \quad \mathbf{G}_{0}=\mathbf{K}_{0} \times \exp \left(\mathfrak{p}_{0}\right)
$$

the Cartan decompositions of $\mathfrak{g}_{0}$ and $\mathbf{G}_{0}$, respectively, corresponding to $\vartheta$. Then:

$$
\begin{array}{lll}
\mathfrak{l}_{0}=\mathfrak{k}_{00} \oplus \mathfrak{p}_{00}, & \text { with } & \mathfrak{k}_{00}=\mathfrak{k}_{0} \cap \mathfrak{l}_{0}, \quad \mathfrak{p}_{00}=\mathfrak{p}_{0} \cap \mathfrak{l}_{0}, \\
\mathbf{L}_{0}=\mathbf{K}_{00} \times \exp \left(\mathfrak{p}_{00}\right), & \text { with } & \mathbf{K}_{00}=\mathbf{K}_{0} \cap \mathbf{L}_{0} .
\end{array}
$$

Let $\mathbf{S}_{0}$ be the analytic Lie subgroup of $\mathbf{G}_{0}$ generated by $\mathfrak{s}_{0}=\left[\mathfrak{l}_{0}, \mathfrak{l}_{0}\right]$. Then $\mathbf{S}_{0}$ is a closed Lie subgroup of $\mathbf{L}_{0}$ and has a finite center.

Proof. The fact that the Lie subgroup $\mathbf{L}_{0}$ is reductive in the sense of [16] and the validity of the decompositions (3.7) and (3.8) are straightforward consequences of [16, Proposition 7.25], because of the characterization of $\mathbf{L}_{0}$ given in (3.5) of Proposition 3.4.

The last statement follows because $\mathbf{S}_{0}$ is an analytic subgroup of a linear group and has a semisimple Lie algebra (see e.g. [16, Proposition 7.9]).

We conclude this section by proving a theorem about the set of connected components of the isotropy.

Theorem 3.6. Let $M$ be a $\mathbf{G}_{0}$-orbit, with corresponding parabolic CR algebra $\left(\mathfrak{g}_{0}, \mathfrak{q}\right)$. Let $\left(\vartheta, \mathfrak{h}_{0}\right)$ be an adapted Cartan pair. We keep the notation of Propositions 3.2, 3.4 and Corollary 3.5.

Then the Cartan subgroup

$$
\mathbf{H}_{0}=\mathbf{Z}_{\mathbf{G}_{0}}\left(\mathfrak{h}_{0}\right)=\left\{g \in \mathbf{G}_{0} \mid \operatorname{Ad}_{\mathfrak{g}_{0}}(g)(H)=H, \quad \forall H \in \mathfrak{h}_{0}\right\}
$$

of $\mathbf{G}_{0}$ is contained in $\mathbf{L}_{0}$.

Let $\mathfrak{h}_{0}$ be maximally noncompact in $\mathfrak{i}_{0}=\mathfrak{q} \cap \mathfrak{g}_{0}$. Then the intersection $\mathfrak{t}_{0}=$ $\mathfrak{h}_{0} \cap \mathfrak{s}_{0}$ is a maximally noncompact Cartan subalgebra of $\mathfrak{s}_{0}$. The corresponding Cartan subgroup $\mathbf{T}_{0}$ of $\mathbf{S}_{0}$ is given by:

$$
\mathbf{T}_{0}=\mathbf{Z}_{\mathbf{S}_{0}}\left(\mathfrak{t}_{0}\right)=\mathbf{Z}_{\mathbf{S}_{0}}\left(\mathfrak{h}_{0}\right)
$$

Denoting by $\pi_{0}$ the group of connected components, we obtain:

$$
\pi_{0}\left(\mathbf{I}_{0}\right) \simeq \pi_{0}\left(\mathbf{K}_{00}\right) \simeq \pi_{0}\left(\mathbf{L}_{0}\right) \simeq \pi_{0}\left(\frac{\mathbf{H}_{0}}{\mathbf{T}_{0}}\right) \simeq \frac{\mathbf{H}_{0}}{\mathbf{T}_{0} \mathbf{H}_{0}^{0}},
$$

where $\mathbf{H}_{0}^{0}$ is the connected component of the identity of $\mathbf{H}_{0}$. In particular, $\pi_{0}\left(\mathbf{I}_{0}\right)$ is an Abelian group. 
Proof. From $\mathfrak{z}_{0} \subset \mathfrak{h}_{0}$, we have $\mathbf{H}_{0}=\mathbf{Z}_{\mathbf{G}_{0}}\left(\mathfrak{h}_{0}\right) \subset \mathbf{Z}_{\mathbf{G}_{0}}\left(\mathfrak{z}_{0}\right)=\mathbf{L}_{0}$.

Assume that $\mathfrak{h}_{0}$ is maximally noncompact in $\mathfrak{i}_{0}$. We know, see e.g. [16, Proposition 7.90(a)], that $\mathbf{H}_{0}$ intersects all connected components of $\mathbf{L}_{0}$ and hence of $\mathbf{I}_{0}$ and of $\mathbf{K}_{00}$. Take $h_{0} \in \mathbf{H}_{0}$ in the connected component $\mathbf{I}_{0}^{0}$ of the identity of $\mathbf{I}_{0}$. We write $h_{0}=k_{0} \exp \left(X_{0}\right)$ with $k_{0} \in \mathbf{K}_{00}$ and $X_{0} \in \mathfrak{p}_{00}$. By [16, Lemma 7.22], both $k_{0}$ and $\exp \left(X_{0}\right)$ centralize $\mathfrak{h}_{0}$, and hence belong to $\mathbf{H}_{0}$. In particular, $\exp \left(X_{0}\right)$ belongs to the connected component $\mathbf{H}_{0}^{0}$ of $\mathbf{H}_{0}$. We note now that $k_{0}$ belongs to $\mathbf{K}_{0} \cap \mathbf{I}_{0}^{0}$, that is the connected component $\mathbf{K}_{00}^{0}$ of the identity of $\mathbf{K}_{00}$. Since the exponential $\exp : \mathfrak{k}_{00} \rightarrow \mathbf{K}_{00}^{0}$ is surjective, $k_{0}=\exp (Y)$ for some $Y \in \mathfrak{k}_{00}$. Since $\mathfrak{z}_{0}$ is $\vartheta$ invariant, the orthogonal of $\mathfrak{k}_{00} \cap \mathfrak{z}_{0}$ in $\mathfrak{k}_{00}$ for the Killing form of $\mathfrak{g}_{0}$ is contained in the orthogonal $\mathfrak{s}_{0}$ of $\mathfrak{z}_{0}$ in $\mathfrak{l}_{0}$, i.e. $\mathfrak{k}_{00} \cap \mathfrak{z}_{0}^{\perp} \subset \mathfrak{s}_{0}$, so that we have the decomposition:

$$
\mathfrak{k}_{00}=\left(\mathfrak{k}_{00} \cap \mathfrak{s}_{0}\right) \oplus\left(\mathfrak{k}_{00} \cap \mathfrak{z}_{0}\right) .
$$

Let $Y=S+Z$, with $S \in \mathfrak{k}_{00} \cap \mathfrak{s}_{0}$ and $Z \in \mathfrak{k}_{00} \cap \mathfrak{z}_{0}$. Then $k_{0}=\exp (S) \exp (Z)$. Since $\exp (Z) \in \mathbf{H}_{0}^{0}$, we obtain that $\exp (S) \in \mathbf{S}_{0} \cap \mathbf{H}_{0}=\mathbf{T}_{0}$ and hence:

$$
h_{0}=\exp (S)\left(\exp (Z) \exp \left(X_{0}\right)\right)
$$

with $\exp (S) \in \mathbf{T}_{0}$, and $\left(\exp (Z) \exp \left(X_{0}\right)\right) \in \mathbf{H}_{0}^{0}$.

Finally, we note that $\pi_{0}\left(\mathbf{I}_{0}\right)$ is Abelian, being a quotient of the Abelian group $\mathbf{H}_{0}$. The proof is complete.

\section{Adapted Weyl chambers}

We keep the notation of Section 3. Let $\left(\vartheta, \mathfrak{h}_{0}\right)$ be a Cartan pair adapted to the parabolic $C R$ algebra $\left(\mathfrak{g}_{0}, \mathfrak{q}\right)$, and $\mathfrak{g}_{0}=\mathfrak{k}_{0} \oplus \mathfrak{p}_{0}$ the Cartan decomposition corresponding to $\vartheta$. We still denote by $\vartheta$ its $\mathbb{C}$-linear extension to $\mathfrak{g}$ and by $\sigma$ and $\tau$ the conjugations of $\mathfrak{g}$ with respect to its real form $\mathfrak{g}_{0}$ and its compact form $\mathfrak{u}=\mathfrak{k}_{0} \oplus i \mathfrak{p}_{0}$, respectively. The $\mathbb{C}$-linear map $\vartheta$ and the anti- $\mathbb{C}$-linear maps $\sigma$ and $\tau$ pairwise commute and:

$$
\tau=\vartheta \circ \sigma=\sigma \circ \vartheta, \quad \sigma=\vartheta \circ \tau=\tau \circ \vartheta, \quad \vartheta=\sigma \circ \tau=\tau \circ \sigma
$$

Let $\mathcal{R}$ be the root system of $\mathfrak{g}$ with respect to $\mathfrak{h}=\mathfrak{h}_{0}^{\mathbb{C}}$. Set

$$
\mathfrak{h}_{0}^{+}=\mathfrak{h}_{0} \cap \mathfrak{k}_{0}, \quad \mathfrak{h}_{0}^{-}=\mathfrak{h}_{0} \cap \mathfrak{p}_{0}, \quad \mathfrak{h}_{\mathbb{R}}=\mathfrak{h}_{0}^{-} \oplus i \mathfrak{h}_{0}^{+}
$$

The root system $\mathcal{R}$ is a subset of the dual $\mathfrak{h}_{\mathbb{R}}^{*}$ of $\mathfrak{h}_{\mathbb{R}}$. The subspace $\mathfrak{h}_{\mathbb{R}}$ of $\mathfrak{g}$ is $\vartheta$, $\sigma$ and $\tau$-invariant. Hence the corresponding involutions of $\mathfrak{h}_{\mathbb{R}}^{*}$ define involutions of $\mathcal{R}$, that are given by:

$$
\sigma^{*}(\alpha)=\bar{\alpha}, \quad \tau^{*}(\alpha)=-\alpha, \quad \vartheta^{*}(\alpha)=-\bar{\alpha} .
$$


Definition 4.1. We denote by $\mathcal{R}_{\text {re }}$ the set of real roots in $\mathcal{R}$, i.e. those for which $\bar{\alpha}=\alpha$, by $\mathcal{R}_{\text {im }}$ the set of imaginary roots in $\mathcal{R}$, i.e. those for which $\bar{\alpha}=-\alpha$, and by $\mathcal{R}_{\mathrm{cpx}}$ the set of complex roots in $\mathcal{R}$, i.e. those for which $\bar{\alpha} \neq \pm \alpha$.

Lemma 4.2. Let $\left(\mathfrak{g}_{0}, \mathfrak{q}\right)$ be a parabolic CR algebra and $\left(\vartheta, \mathfrak{h}_{0}\right)$ a Cartan pair adapted to $\left(\mathfrak{g}_{0}, \mathfrak{q}\right)$. Then the reductive factor $\mathfrak{q}^{r}$ in the decomposition (2.8) with respect to the Cartan subalgebra $\mathfrak{h}=\mathbb{C} \otimes_{\mathbb{R}} \mathfrak{h}_{0}$ is

$$
\mathfrak{q}^{r}=\mathfrak{q} \cap \tau(\mathfrak{q}) .
$$

Proof. Indeed, if $\mathcal{R}$ is the root system of $\mathfrak{g}$ corresponding to the Cartan subalgebra $\mathfrak{h}$, then $\tau$ transforms the eigenspace $\mathfrak{g}^{\alpha}$ of $\alpha \in \mathcal{R}$ into $\mathfrak{g}^{-\alpha}$.

We have the following:

Lemma 4.3. Let $\left(\mathfrak{g}_{0}, \mathfrak{q}\right)$ be a parabolic $C R$ algebra and $\left(\vartheta, \mathfrak{h}_{0}\right)$ a Cartan pair adapted to $\left(\mathfrak{g}_{0}, \mathfrak{q}\right)$. Then:

(1) There exists a fit Weyl chamber $C^{\prime} \in \mathfrak{C}(\mathcal{R}, \mathcal{Q})$ such that, if $\prec$ is the partial order in $\mathfrak{h}_{\mathbb{R}}^{*}$ defined by $C^{\prime}, \mathcal{B}^{\prime}$ the basis of $C^{\prime}$-positive simple roots, and $\Phi^{\prime}=$ $\mathcal{Q}^{n} \cap \mathcal{B}^{\prime}$, the two following equivalent conditions are satisfied:

$$
\begin{aligned}
& \text { if } \alpha \in \mathcal{R}_{\mathrm{cpx}}, \alpha \succ 0 \text { and } \bar{\alpha} \prec 0 \text {, then } \alpha \text { and }-\bar{\alpha} \text { both belong to } \mathcal{Q}^{n}, \\
& \bar{\alpha} \succ 0 \text { for all } \alpha \in \mathcal{B}^{\prime} \backslash\left(\Phi^{\prime} \cup \mathcal{R}_{\mathrm{im}}\right) \text {. }
\end{aligned}
$$

(2) There exists a fit Weyl chamber $C^{\prime \prime} \in \mathfrak{C}(\mathcal{R}, \mathcal{Q})$ such that, if $\prec$ is the partial order in $\mathfrak{h}_{\mathbb{R}}^{*}$ defined by $C^{\prime \prime}, \mathcal{B}^{\prime \prime}$ the basis of $C^{\prime \prime}$-positive simple roots, and $\Phi^{\prime \prime}=$ $\mathcal{Q}^{n} \cap \mathcal{B}^{\prime \prime}$, the two following equivalent conditions are satisfied:

$$
\begin{aligned}
& \text { if } \alpha \in \mathcal{R}_{\mathrm{cpx}}, \alpha \succ 0 \text { and } \bar{\alpha} \succ 0, \text { then } \alpha \text { and } \bar{\alpha} \text { both belong to } \mathcal{Q}^{n}, \\
& \bar{\alpha} \prec 0 \text { for all } \alpha \in \mathcal{B}^{\prime \prime} \backslash\left(\Phi^{\prime \prime} \cup \mathcal{R}_{\mathrm{re}}\right) \text {. }
\end{aligned}
$$

Proof. For $C \in \mathfrak{C}(\mathcal{R})$, we denote by $v(C)$ the number of $C$-positive roots $\alpha$ for which also $\bar{\alpha}$ is $C$-positive. Choose $C^{\prime} \in \mathfrak{C}(\mathcal{R}, \mathcal{Q})$ in such a way that:

$$
v\left(C^{\prime}\right)=\max _{C \in \mathfrak{C}(\mathcal{R}, \mathcal{Q})} v(C) .
$$

If there was a complex $C^{\prime}$-positive root $\alpha \in \mathcal{B}^{\prime} \backslash \Phi^{\prime}$ with a $C^{\prime}$-negative $\bar{\alpha}$, the symmetry $s_{\alpha}$ would transform $C^{\prime}$ into a $C^{*}$ that still belongs to $\mathfrak{C}(\mathcal{R}, \mathcal{Q})$. Since the set $\mathcal{R}^{+}\left(C^{*}\right)$ of $C^{*}$-positive roots is $\left(\mathcal{R}^{+}\left(C^{\prime}\right) \backslash\{\alpha\}\right) \cup\{-\alpha\}$, we should have $v\left(C^{*}\right)=v\left(C^{\prime}\right)+1$ and hence a contradiction. This shows that (4.6) is valid for $C^{\prime}$.

Next we observe that obviously (4.5) implies (4.6). Vice versa, assume that (4.6) is true and let $\alpha=\sum_{\beta \in \mathcal{B}^{\prime}} k_{\alpha}^{\beta} \beta$ be a complex $C^{\prime}$-positive root with a $C^{\prime}$ negative $\bar{\alpha}$. Then $\bar{\beta}$ should be $C^{\prime}$-negative for some complex $\beta$ in $\operatorname{supp}(\alpha)$, and hence $\operatorname{supp}(\alpha) \cap \Phi^{\prime} \neq \emptyset$ by (4.6), implying that $\alpha \in \mathcal{Q}^{n}$. The same argument, applied to the complex $C^{\prime}$-positive $\operatorname{root}(-\bar{\alpha})$, shows then that also $(-\bar{\alpha}) \in \mathcal{Q}^{n}$.

Similarly, by taking a $C^{\prime \prime} \in \mathfrak{C}(\mathcal{R}, \mathcal{Q})$ that minimizes $v(C)$ in $\mathfrak{C}(\mathcal{R}, \mathcal{Q})$ we obtain a fit Weyl chamber that satisfies the conditions (4.7) and (4.8). 
Definition 4.4. Let $\left(\vartheta, \mathfrak{h}_{0}\right)$ be a Cartan pair adapted to a parabolic $C R$ algebra $\left(\mathfrak{g}_{0}, \mathfrak{q}\right), \mathcal{R}$ the root system of $\mathfrak{g}$ with respect to the complexification $\mathfrak{h}$ of $\mathfrak{h}_{0}, \mathcal{Q}$ the parabolic set of $\mathfrak{q}$ in $\mathcal{R}$. A fit Weyl chamber $C$ for $\mathcal{Q}$ is:

$S$-fit for $\left(\mathfrak{g}_{0}, \mathfrak{q}\right)$ if $C$ satisfies the equivalent conditions (4.5), (4.6);

$V$-fit for $\left(\mathfrak{g}_{0}, \mathfrak{q}\right)$ if $C$ satisfies the equivalent conditions (4.7), (4.8).

\section{5. $\mathbf{G}_{0}$-equivariant fibrations}

In this section we shall discuss $\mathbf{G}_{0}$-equivariant fibrations of $\mathbf{G}_{0}$-orbits. We begin by proving that their structures of $\mathbf{G}_{0}$-homogeneous space is completely determined by the Lie algebras of their isotropy subgroups.

Theorem 5.1. Let $M, M^{\prime}$ be $\mathbf{G}_{0}$-orbits, corresponding to $C R$ algebras $\left(\mathfrak{g}_{0}, \mathfrak{q}\right)$, $\left(\mathfrak{g}_{0}, \mathfrak{q}^{\prime}\right)$, where $\mathfrak{q}, \mathfrak{q}^{\prime}$ are two complex parabolic subalgebras of $\mathfrak{g}$. Let $\mathfrak{i}_{0}=\mathfrak{q} \cap \mathfrak{g}_{0}$ and $\mathfrak{i}_{0}^{\prime}=\mathfrak{q}^{\prime} \cap \mathfrak{g}_{0}$ be the Lie subalgebras of the isotropy subgroups $\mathbf{I}_{0}$ and $\mathbf{I}_{0}^{\prime}$ of $M$ and $M^{\prime}$, respectively. Then:

$$
\mathfrak{i}_{0}^{\prime} \subset \mathfrak{i}_{0} \Longrightarrow \mathbf{I}_{0}^{\prime} \subset \mathbf{I}_{0}
$$

and therefore, if $\mathfrak{i}_{0}^{\prime} \subset \mathfrak{i}_{0}$, there is a canonical $\mathbf{G}_{0}$-equivariant fibration:

$$
M^{\prime} \stackrel{\phi}{\longrightarrow} M
$$

Assume that $\mathfrak{i}_{0}^{\prime} \subset \mathfrak{i}_{0}$. We have:

(1) If $\mathfrak{i}_{0}^{\prime}$ contains a Cartan subalgebra $\mathfrak{h}_{0}$ that is maximally noncompact as a Cartan subalgebra of $\mathfrak{i}_{0}$, then the fiber $F$ of (5.2) is connected.

(2) The projection $\phi$ is a CR map if and only if $\mathfrak{q}^{\prime} \subset \mathfrak{q}$.

(3) The projection $\phi$ is a CR submersion, and hence (5.2) is a CR fibration, if and only if $\mathfrak{q}=\mathfrak{q}^{\prime}+(\mathfrak{q} \cap \overline{\mathfrak{q}})$.

Proof. Assume that $\mathfrak{i}_{0}^{\prime} \subset \mathfrak{i}_{0}$. To prove that $\mathbf{I}_{0}^{\prime} \subset \mathbf{I}_{0}$, it suffices to prove that each connected component of $\mathbf{I}_{0}^{\prime}$ intersects $\mathbf{I}_{0}$.

Fix a Cartan pair $\left(\vartheta, \mathfrak{h}_{0}\right)$ adapted to both $\left(\mathfrak{g}_{0}, \mathfrak{q}^{\prime}\right)$, and $\left(\mathfrak{g}_{0}, \mathfrak{q}\right)$. This can be obtained by first taking the Cartan involution $\vartheta$ associated to a maximal compact subalgebra $\mathfrak{k}_{0}$ of $\mathfrak{g}_{0}$, with $\mathfrak{k}_{00}^{\prime}=\mathfrak{k}_{0} \cap \mathfrak{i}_{0}^{\prime}$ and $\mathfrak{k}_{00}=\mathfrak{k}_{0} \cap \mathfrak{i}_{0}$ maximal compact in $\mathfrak{i}_{0}^{\prime}$ and $\mathfrak{i}_{0}$, respectively, and then choosing any $\vartheta$-invariant Cartan subalgebra $\mathfrak{h}_{0}$ of $\mathfrak{i}_{0}^{\prime}$.

Consider the centers $\mathfrak{z}_{0}^{\prime}$ of $\mathfrak{l}_{0}^{\prime}=\mathfrak{i}_{0}^{\prime} \cap \vartheta\left(\mathfrak{i}_{0}^{\prime}\right)$ and $\mathfrak{z}_{0}$ of $\mathfrak{l}_{0}=\mathfrak{i}_{0} \cap \vartheta\left(\mathfrak{i}_{0}\right)$. Since $\mathfrak{z}_{0} \subset \mathfrak{l}_{0}^{\prime}$, we have $\mathfrak{z}_{0}^{\prime} \supset \mathfrak{z}_{0}$. Thus, by (3.5), $\mathbf{L}_{0}^{\prime}=\mathbf{Z}_{\mathbf{G}_{0}}\left(\mathfrak{z}_{0}^{\prime}\right) \subset \mathbf{Z}_{\mathbf{G}_{0}}\left(\mathfrak{z}_{0}\right)=\mathbf{L}_{0}$. By (3.4), $\mathbf{L}_{0}^{\prime}$ is a deformation retract of $\mathbf{I}_{0}^{\prime}$. Then each connected component of $\mathbf{I}_{0}^{\prime}$ intersects $\mathbf{L}_{0}^{\prime}$ and thus also $\mathbf{I}_{0}$. This proves (5.1).

(1) Assume that $\mathfrak{h}_{0}$ is a Cartan subalgebra of $\mathfrak{i}_{0}^{\prime}$ that is maximally noncompact both in $\mathfrak{i}_{0}^{\prime}$ and in $\mathfrak{i}_{0}$, and consider the corresponding Cartan subgroup $\mathbf{H}_{0}=\mathbf{Z}_{\mathbf{G}_{0}}\left(\mathfrak{h}_{0}\right)$. By [16, Proposition 7.90], each connected component of $\mathbf{L}_{0}$ and of $\mathbf{L}_{0}^{\prime}$, and, because 
of (3.4), of $\mathbf{I}_{0}^{\prime}$ and of $\mathbf{I}_{0}$, intersects $\mathbf{H}_{0}$. Being $\mathbf{H}_{0} \subset \mathbf{L}_{0}^{\prime} \subset \mathbf{L}_{0}$, this shows that each connected component of $\mathbf{I}_{0}$ contains points of $\mathbf{I}_{0}^{\prime}$, and hence fore $\mathbf{I}_{0} / \mathbf{I}_{0}^{\prime}$ is connected.

(2) Let $\mathbf{I}_{0}^{\prime}$ and $\mathbf{I}_{0}$ be the isotropy subgroups of $M^{\prime}$ and $M$ at the points $x_{0}^{\prime}$ and $x_{0}=\phi\left(x_{0}^{\prime}\right)$, respectively. Denote by $d \pi^{\prime}: \mathfrak{g} \rightarrow T_{x_{0}^{\prime}}^{\mathbb{C}} M^{\prime}$ and by $d \pi: \mathfrak{g} \rightarrow T_{x_{0}}^{\mathbb{C}} M$ the complexification of the differential at the identity of the action of $\mathbf{G}_{0}$ on $M^{\prime}$ and $M$, with base points $x_{0}^{\prime}$ and $x_{0}$. By (1.2) $\mathfrak{q}^{\prime}=d \pi^{\prime-1}\left(T_{x_{0}^{\prime}}^{0,1} M^{\prime}\right)$ and $\mathfrak{q}=d \pi^{-1}\left(T_{x_{0}}^{0,1} M\right)$. Let $d \phi_{x_{0}^{\prime}}^{\mathbb{C}}$ be the complexification of the differential of $\phi$ at $x_{0}^{\prime}$. When $\phi$ is a $C R$ map, we have:

$$
d \pi\left(\mathfrak{q}^{\prime}\right)=d \phi_{x_{0}^{\prime}}^{\mathbb{C}}\left(d \pi^{\prime}\left(\mathfrak{q}^{\prime}\right)\right)=d \phi_{x_{0}^{\prime}}^{\mathbb{C}}\left(T_{x_{0}^{\prime}}^{0,1} M^{\prime}\right) \subset T_{x_{0}}^{0,1} M=d \pi(\mathfrak{q}) .
$$

Since $\mathfrak{q}=(d \pi)^{-1}(d \pi(\mathfrak{q}))$, this implies that $\mathfrak{q}^{\prime} \subset \mathfrak{q}$. Vice versa, when $\mathfrak{q}^{\prime} \subset \mathfrak{q}$, the map $\phi: M^{\prime} \rightarrow M$ is $C R$, being the restriction of the $\mathbf{G}$-equivariant holomorphic projection $X^{\prime}=\mathbf{G} / \mathbf{Q}^{\prime} \rightarrow \mathbf{G} / \mathbf{Q}=X$.

Finally, (3) is a consequence of [21, Lemma 4.5].

Remark 5.2. By Theorem 5.1, orbits $M$ and $M^{\prime}$, corresponding to parabolic $C R$ algebras $\left(\mathfrak{g}_{0}, \mathfrak{q}\right)$ and $\left(\mathfrak{g}_{0}, \mathfrak{q}^{\prime}\right)$ with $\mathfrak{q}^{\prime} \cap \overline{\mathfrak{q}}^{\prime}=\mathfrak{q} \cap \overline{\mathfrak{q}}$, are $\mathbf{G}_{0}$-equivariantly diffeomorphic. Thus, having fixed the real subalgebra $\mathfrak{i}_{0}$, we can regard the complex parabolic $\mathfrak{q}$ 's with $\mathfrak{q} \cap \mathfrak{g}_{0}=\mathfrak{i}_{0}$ as defining different $\mathbf{G}_{0}$-invariant $C R$ structures on a same $\mathbf{G}_{0^{-}}$ homogeneous smooth manifold $M$, each corresponding to a different $C R$-generic embedding of $M$ into a complex flag manifold.

In the next theorem we construct a somehow minimal $C R$ structure on a $\mathbf{G}_{0^{-}}$ orbit $M$. Note that the subalgebra $\mathfrak{q}_{w}$ of the theorem below is equal to the $\mathfrak{q}^{\prime}$ used in the proof of Proposition 3.4.

Theorem 5.3. Let $\left(\mathfrak{g}_{0}, \mathfrak{q}\right)$ be a parabolic CR algebra. Then:

$$
\mathfrak{q}_{w}=\mathfrak{q}^{n}+\mathfrak{q} \cap \overline{\mathfrak{q}}
$$

is a complex parabolic subalgebra of $\mathfrak{g}$, contained in $\mathfrak{q}$. It is the minimal complex parabolic subalgebra of $\mathfrak{g}$ with the properties that:

$$
\mathfrak{q}_{w} \cap \overline{\mathfrak{q}}_{w}=\mathfrak{q} \cap \overline{\mathfrak{q}} \quad \text { and } \quad \mathfrak{q}_{w} \subset \mathfrak{q} .
$$

Any Cartan pair $\left(\vartheta, \mathfrak{h}_{0}\right)$ adapted to $\left(\mathfrak{g}_{0}, \mathfrak{q}\right)$ is also adapted to $\left(\mathfrak{g}_{0}, \mathfrak{q}_{w}\right)$. Fix a Cartan pair $\left(\vartheta, \mathfrak{h}_{0}\right)$ adapted to $\left(\mathfrak{g}_{0}, \mathfrak{q}\right)$ and hence also to $\left(\mathfrak{g}_{0}, \mathfrak{q}_{w}\right)$. Let $\tau$ be the conjugation with respect to the $\vartheta$-invariant compact form of $\mathfrak{g}$ (see (3.1)), and set

$$
\mathfrak{q}^{r}=\mathfrak{q} \cap \tau(\mathfrak{q}), \quad \mathfrak{q}_{w}^{r}=\mathfrak{q}_{w} \cap \tau\left(\mathfrak{q}_{w}\right) .
$$

Then we have:

$$
\begin{aligned}
\mathfrak{q}_{w} & =\mathfrak{q}^{n}+\mathfrak{q}^{r} \cap \overline{\mathfrak{q}}, \\
\mathfrak{q}_{w}^{n} & =\mathfrak{q}^{n}+\mathfrak{q}^{r} \cap \overline{\mathfrak{q}}^{n}, \\
\mathfrak{q}_{w}^{r}=\overline{\mathfrak{q}}_{w}^{r} & =\mathfrak{q}_{w}^{r} \cap \overline{\mathfrak{q}}_{w}^{r}=\mathfrak{q}^{r} \cap \overline{\mathfrak{q}}^{r} .
\end{aligned}
$$


Let $M_{w}$ be the $\mathbf{G}_{0}$-orbit corresponding to $\left(\mathfrak{g}_{0}, \mathfrak{q}_{w}\right)$. Then the canonical $\mathbf{G}_{0}$-equivariant fibration $M_{w} \rightarrow M$ is a CR map and a smooth diffeomorphism.

Proof. Let $\left(\vartheta, \mathfrak{h}_{0}\right)$ be any Cartan pair adapted to $(\mathfrak{g}, \mathfrak{q})$. Let $\mathcal{R}$ be the root system of $\mathfrak{g}$, with respect to the complexification $\mathfrak{h}$ of $\mathfrak{h}_{0}$. We fix an $A \in \mathfrak{h}_{\mathbb{R}}$ that defines the parabolic set $\mathcal{Q}$ of $\mathfrak{q}$, i.e. such that (cf. e.g. [8, VIII Section 4]: $A$ is any element of the interior of the facet associated to $\mathfrak{q}$ and $\mathfrak{h})$ :

$$
\mathcal{Q}=\{\alpha \in \mathcal{R} \mid \alpha(A) \geq 0\} .
$$

If $\epsilon>0$ is so small that $\alpha(A)>\epsilon|\bar{\alpha}(A)|$ for all $\alpha \in \mathcal{Q}^{n}$, then $\mathfrak{q}_{w}$ is the complex parabolic Lie subalgebra of $\mathfrak{g}$ that corresponds to the parabolic set:

$$
\mathcal{Q}_{w}=\{\alpha \in \mathcal{R} \mid \alpha(A+\epsilon \bar{A}) \geq 0\} .
$$

This observation easily yields (5.4), (5.6), (5.7), (5.8). The last statement follows from (5.4), Theorem 5.1, and Remark 5.2. The fact that $\mathfrak{q}_{w}$ is minimal with the properties of (5.4) follows from (5.8): in fact any parabolic subalgebra containing $\mathfrak{q} \cap \overline{\mathfrak{q}}$ must contain the reductive subalgebra $\mathfrak{q}^{r} \cap \overline{\mathfrak{q}}^{r}$.

Definition 5.4. The parabolic $C R$ algebra $\left(\mathfrak{g}_{0}, \mathfrak{q}_{w}\right)$, and the corresponding $\mathbf{G}_{0}$-orbit $M_{w}$, are called the $C R$-weakening of $\left(\mathfrak{g}_{0}, \mathfrak{q}\right)$, and of $M$, respectively.

Remark 5.5. In [28, Section 9.1] the orbits $M$ corresponding to parabolic $C R$ algebras $\left(\mathfrak{g}_{0}, \mathfrak{q}\right)$ with $\mathfrak{q}^{r}=\overline{\mathfrak{q}}^{r}$ are called polarized. Thus the $C R$ weakening of $M$ is polarized, and, vice versa, if $M$ is polarized, $M$ coincides with its $C R$ weakening $M_{w}$.

We have:

Lemma 5.6. Let $\left(\mathfrak{g}_{0}, \mathfrak{q}\right)$ be a polarized parabolic $C R$ algebra, i.e. assume that $\mathfrak{q}$ contains a maximal reductive subalgebra $\mathfrak{q}^{r}$ with $\mathfrak{q}^{r}=\overline{\mathfrak{q}}^{r}$. Then $\left(\mathfrak{g}_{0}, \mathfrak{q}\right)$ is either totally real, or weakly degenerate.

Proof. Let $\left(\vartheta, \mathfrak{h}_{0}\right)$ be a Cartan pair adapted to $\left(\mathfrak{g}_{0}, \mathfrak{q}\right)$ and $\mathcal{R}$ the root system of $\mathfrak{g}$ with respect to the complexification $\mathfrak{h}$ of $\mathfrak{h}_{0}$. We fix an $S$-fit Weyl chamber for $\left(\mathfrak{g}_{0}, \mathfrak{q}\right)$. By the assumption, the parabolic set $\mathcal{Q}$ of $\mathfrak{q}$ has the property that $\mathcal{Q}^{r}=\overline{\mathcal{Q}}^{r}$. This is equivalent to the fact that

$$
\operatorname{supp}(\bar{\alpha}) \cap \Phi \neq \emptyset \quad \forall \alpha \in \mathcal{Q}^{n}
$$

where $\Phi$ is the set of $C$-positive simple roots in $\mathcal{Q}$. If $\bar{\alpha} \succ 0$ for all $\alpha \in \Phi$, then $\overline{\mathcal{Q}}^{n}=\mathcal{Q}^{n}$, and $\mathfrak{q}$ is totally real. Assume that there is $\alpha \in \Phi$ with $\bar{\alpha} \prec 0$ and consider the parabolic $\mathfrak{q}^{\prime}=\mathfrak{q}_{\Psi}$, with $\Psi=\Phi \backslash\{\alpha\}$. Then

$$
\mathcal{Q}_{\Psi} \supset \mathcal{Q} \quad \text { and } \quad \mathcal{Q}_{\Psi} \backslash \mathcal{Q}=\{\beta \in \mathcal{R} \mid \beta \prec 0, \operatorname{supp}(\beta) \cap \Phi=\{\alpha\}\} .
$$

If $\beta \in\left(\mathcal{Q}_{\Psi} \backslash \mathcal{Q}\right)$, then $\bar{\beta} \succ 0$. Indeed, $\operatorname{supp}(\bar{\alpha}) \cap \Phi \neq \emptyset$, and $\operatorname{supp}(\bar{\gamma}) \cap \Phi=\emptyset$ if $\gamma$ is a $C$-positive simple root not belonging to $\Phi$. Thus, the decomposition of $\bar{\beta}$ 
into a linear combination of $C$-positive simple roots contains some element of $\Phi$ with a positive coefficient and hence is positive. This shows that $\left(\mathcal{Q}_{\Psi} \backslash \mathcal{Q}\right) \subset \overline{\mathcal{Q}}$. Therefore $\mathcal{Q}_{\Psi} \subset \mathcal{Q} \cup \overline{\mathcal{Q}}$. Thus we obtained $\mathfrak{q} \subsetneq \mathfrak{q}_{\Psi} \subset \mathfrak{q}+\overline{\mathfrak{q}}$, showing that $\left(\mathfrak{g}_{0}, \mathfrak{q}\right)$ is weakly degenerate.

From Remark 5.5 and Lemma 5.6, and the characterization of the $C R$-weakening in the proof of Theorem 5.3, we obtain:

Proposition 5.7. Let $\left(\mathfrak{g}_{0}, \mathfrak{q}\right)$ be a parabolic CR algebra, and $\left(\mathfrak{g}_{0}, \mathfrak{q}_{w}\right)$ its CR-weakening. Then $\left(\mathfrak{g}_{0}, \mathfrak{q}_{w}\right)$ is either totally real, or weakly degenerate.

Let $\left(\vartheta, \mathfrak{h}_{0}\right)$ be an adapted Cartan pair for $\left(\mathfrak{g}_{0}, \mathfrak{q}\right)$, and hence also for $\left(\mathfrak{g}_{0}, \mathfrak{q}_{w}\right)$. Denote by $\mathcal{R}$ the root system of $\mathfrak{g}$ with respect to the complexification $\mathfrak{h}$ of $\mathfrak{h}_{0}$, and by $\mathcal{Q}, \mathcal{Q}_{w}$ the parabolic sets of $\mathfrak{q}, \mathfrak{q}_{w}$, respectively. Then:

(1) $C \in \mathfrak{C}(\mathcal{R}, \mathcal{Q})$ is $S$-fit for $\left(\mathfrak{g}_{0}, \mathfrak{q}\right)$ if and only if it is $S$-fit for $\left(\mathfrak{g}_{0}, \mathfrak{q}_{w}\right)$.

(2) Let $\mathcal{B}$ be the basis of $C$-positive simple roots for an $S$-fit $C \in \mathfrak{C}(\mathcal{R}, \mathcal{Q})$, and $\Phi=\mathcal{B} \cap \mathcal{Q}^{n}$, so that $\mathfrak{q}=\mathfrak{q}_{\Phi}$. Then $\mathfrak{q}_{w}=\mathfrak{q}_{\Phi_{w}}$ with

$$
\Phi_{w}=\Phi \cup\{\alpha \in \mathcal{B} \mid \bar{\alpha} \succ 0, \operatorname{supp}(\bar{\alpha}) \cap \Phi \neq \emptyset\}
$$

We introduce the following:

Definition 5.8. Let $M$ be a $\mathbf{G}_{0}$-homogeneous $C R$ manifold with associated $C R$ algebra $\left(\mathfrak{g}_{0}, \mathfrak{q}\right)$. A strengthening of the $C R$ structure of $M$ is the datum of a complex Lie subalgebra $\mathfrak{q}^{\prime}$ with:

$$
\mathfrak{q}^{\prime} \cap \overline{\mathfrak{q}}^{\prime}=\mathfrak{q} \cap \overline{\mathfrak{q}} \quad \text { and } \quad \mathfrak{g} \supset \mathfrak{q}^{\prime} \supset \mathfrak{q}
$$

We say that the $\mathbf{G}_{0}$-homogeneous $C R$ structure defined by $\left(\mathfrak{g}_{0}, \mathfrak{q}\right)$ is maximal if $\mathfrak{q}^{\prime}=\mathfrak{q}$ for all complex Lie subalgebras $\mathfrak{q}^{\prime}$ of $\mathfrak{g}$ satisfying (5.10).

If $\left(\mathfrak{g}_{0}, \mathfrak{q}\right)$ is a parabolic $C R$ algebra and $M_{s}$ is the $\mathbf{G}_{0}$-orbit associated to a strengthening $\left(\mathfrak{g}_{0}, \mathfrak{q}_{s}\right)$ of $\left(\mathfrak{g}_{0}, \mathfrak{q}\right)$, then the $\mathbf{G}_{0}$-equivariant map $M \rightarrow M_{S}$ is a diffeomorphism and a $C R$ map. We have:

Proposition 5.9. Let $M$ be the $\mathbf{G}_{0}$-orbit associated to the parabolic CR algebra $\left(\mathfrak{g}_{0}, \mathfrak{q}\right)$. Fix an adapted Cartan pair $\left(\vartheta, \mathfrak{h}_{0}\right)$ and let $\mathfrak{q}=\mathfrak{q}_{\Phi}$ for a system $\Phi$ of $C$-positive simple roots of an $S$-fit Weyl chamber $C$. Then:

(1) The necessary and sufficient condition for the CR structure defined by $\left(\mathfrak{g}_{0}, \mathfrak{q}_{\Phi}\right)$ to be maximal is that:

$$
\alpha \in \Phi \text { and } \bar{\alpha} \succ 0 \Longrightarrow \operatorname{supp}(\bar{\alpha}) \cap \Phi \subset\{\alpha\}
$$

(2) There are maximal $\mathbf{G}_{0}$-homogeneous $C R$ structures $\left(\mathfrak{g}_{0}, \mathfrak{q}^{\prime}\right)$ on $M$, and for each of them $\mathfrak{q}^{\prime}=\mathfrak{q}_{\Psi}$ for some system of simple roots $\Psi \subset \Phi$. 
Proof. Let $\alpha \in \Phi$ and set $\Psi=\Phi \backslash\{\alpha\}$. If $\bar{\alpha} \prec 0$, then $-\alpha \in\left(\mathcal{Q}_{\Psi} \cap \overline{\mathcal{Q}}_{\Psi}\right) \backslash\left(\mathcal{Q}_{\Phi} \cap\right.$ $\overline{\mathcal{Q}}_{\Phi}$ ), and hence $\mathfrak{q}_{\Psi}$ does not satisfy (5.10). If $\bar{\alpha} \succ 0$ and $\operatorname{supp}(\bar{\alpha}) \cap \Phi \subset\{\alpha\}$, again $-\alpha \in\left(\mathcal{Q}_{\Psi} \cap \overline{\mathcal{Q}}_{\Psi}\right) \backslash\left(\mathcal{Q}_{\Phi} \cap \overline{\mathcal{Q}}_{\Phi}\right)$, showing that also in this case $\mathfrak{q}_{\Psi}$ does not satisfy (5.10). Since all complex subalgebras $\mathfrak{q}^{\prime}$ that satisfy (5.10) are of the form $\mathfrak{q}^{\prime}=\mathfrak{q}_{\Psi}$ for some $\Psi \subset \Phi$, this proves that (5.11) is a necessary condition.

Vice versa, if there is $\alpha \in \Phi$ with $\bar{\alpha} \succ 0$ and $\operatorname{supp}(\bar{\alpha}) \cap \Phi \not \subset\{\alpha\}$, then, for $\Psi=\Phi \backslash\{\alpha\}$, the complex subalgebra $\mathfrak{q}_{\Psi}$ satisfies $(5.10)$, and hence $\left(\mathfrak{g}_{0}, \mathfrak{q}_{\Phi}\right)$ is not maximal. This proves (1).

To prove (2), it suffices to take any maximal element of the set of all complex Lie subalgebras $\mathfrak{q}^{\prime}$ with $\mathfrak{q} \subset \mathfrak{q}^{\prime} \subset \mathfrak{g}$ and $\mathfrak{q}^{\prime} \cap \overline{\mathfrak{q}}^{\prime}=\mathfrak{q} \cap \overline{\mathfrak{q}}$.

We conclude this section by proving a theorem that describes the structure of the fiber $F$ of a $\mathbf{G}_{0}$-equivariant $C R$ fibration.

Theorem 5.10. Let $M, M^{\prime}$ be $\mathbf{G}_{0}$-orbits, corresponding to the parabolic CR algebras $\left(\mathfrak{g}_{0}, \mathfrak{q}\right),\left(\mathfrak{g}_{0}, \mathfrak{q}^{\prime}\right)$. Assume that $\mathfrak{q}^{\prime} \subset \mathfrak{q}$, so that $\mathbf{I}_{0} \supset \mathbf{I}_{0}^{\prime}$ for the isotropy subgroups with Lie algebras $\mathfrak{i}_{0}=\mathfrak{q} \cap \mathfrak{g}_{0}$ and $\mathfrak{i}_{0}^{\prime}=\mathfrak{q}^{\prime} \cap \mathfrak{g}_{0}$.

Fix a Cartan pair $\left(\vartheta, \mathfrak{h}_{0}\right)$, adapted to $\left(\mathfrak{g}_{0}, \mathfrak{q}^{\prime}\right)$, and hence also to $\left(\mathfrak{g}_{0}, \mathfrak{q}\right)$.

Consider the canonical $\mathbf{G}_{0}$-equivariant fibration $M^{\prime} \rightarrow M$, with typical fiber $F=\mathbf{I}_{0} / \mathbf{I}_{0}^{\prime}$. We use the notation of Propositions 3.2 and 3.4.

The fiber $F$ is an $\mathbf{I}_{0}$-homogeneous $C R$ manifold. Its associated CR algebra $\left(\mathfrak{i}_{0}, \overline{\mathfrak{q}} \cap \mathfrak{q}^{\prime}\right)$ is the semidirect product of the CR algebras $\left(\mathfrak{l}_{0}, \mathfrak{q}^{\prime} \cap \mathfrak{l}\right)$ and $\left(\mathfrak{n}_{0}, \mathfrak{q}^{\prime} \cap \mathfrak{n}\right)$, where:

(1) $\left(\mathfrak{l}_{0}, \mathfrak{q}^{\prime} \cap \mathfrak{l}\right)$ is a parabolic $C R$ algebra;

(2) $\left(\mathfrak{n}_{0}, \mathfrak{q}^{\prime} \cap \mathfrak{n}\right)$ is nilpotent and totally complex, i.e. $\mathfrak{n}=\mathfrak{n}_{0}+\left(\mathfrak{q}^{\prime} \cap \mathfrak{n}\right)$.

The fiber $F$ is $C R$ diffeomorphic to a Cartesian product:

where:

$$
F=F^{\prime} \times F^{\prime \prime}
$$

(1') $F^{\prime}$ has finitely many connected components, each isomorphic to the $\mathbf{L}_{0}^{0}$-orbit in the flag manifold $X^{\prime}=\mathbf{L} /\left(\mathbf{Q}^{\prime} \cap \mathbf{L}\right)$, corresponding to the parabolic $C R$ algebra $\left(\mathfrak{l}_{0}, \mathfrak{q}^{\prime} \cap \mathfrak{l}\right)$. Here we denoted by $\mathbf{L}_{0}^{0}$ the connected component of the identity of $\mathbf{L}_{0}$.

(2') $F^{\prime \prime}$ is a Euclidean complex $\mathbf{N}_{0}$-nilmanifold, with associated CRalgebra $\left(\mathfrak{n}_{0}, \mathfrak{q}^{\prime} \cap\right.$ $\mathfrak{n})$.

If $\mathfrak{q} \subset \mathfrak{q}^{\prime}+\overline{\mathfrak{q}}^{\prime}$, then the fibers of the $\mathbf{G}_{0}$-equivariant fibration $M^{\prime} \rightarrow M$ are complex and simply connected (but not necessarily connected).

Proof. The $C R$ structure of the fiber $F$ is defined by the embedding into the complex flag manifold $X^{\prime \prime}=\mathbf{Q} / \mathbf{Q}^{\prime}$. However, the embedding $F \hookrightarrow X^{\prime \prime}$ is, in general, not $C R$-generic. Thus we begin by considering a natural generic embedding of $F$.

The algebraic subgroup $\mathbf{Q} \cap \overline{\mathbf{Q}}$ decomposes into the semidirect product

$$
\mathbf{Q} \cap \overline{\mathbf{Q}}=\mathbf{L} \ltimes \mathbf{N},
$$

where $\mathbf{N}$ is its unipotent radical and $\mathbf{L}=\mathbf{Q}^{r} \cap \overline{\mathbf{Q}}^{r}$ is reductive. 
The intersection $\mathbf{Q} \cap \overline{\mathbf{Q}}$ contains a Cartan subgroup of $\mathbf{G}$, and therefore is connected. Thus also the groups $\mathbf{L}$ and $\mathbf{N}$ are connected. Their Lie algebras are $\mathfrak{l}$ and $\mathfrak{n}$, respectively. Moreover, $\mathbf{N}$ is also simply connected, being conjugate, in the linear group $\mathbf{G}$, to a group of unipotent upper triangular matrices (see e.g. [15, Section 17.5]).

The connected component $\mathbf{L}_{0}^{0}$ of the identity of $\mathbf{L}_{0}$ is a real form of $\mathbf{L}$. The parabolic subalgebra $\mathfrak{q}^{\prime}$ of $\mathfrak{g}$, containing a Cartan subalgebra of $\mathfrak{l}$, intersects $\mathfrak{l}$ into the complex parabolic subalgebra $\mathfrak{q}^{\prime} \cap \mathfrak{l}$ of $\mathfrak{l}$. The intersection $\mathbf{Q}^{\prime} \cap \mathbf{L}$ is the parabolic subgroup of $\mathbf{L}$ corresponding to $\mathfrak{q}^{\prime} \cap \mathfrak{l}$. The quotient $F^{\prime}=\mathbf{L}_{0} /\left(\mathbf{Q}^{\prime} \cap \mathbf{L}_{0}\right)$ is therefore the union of finitely many copies of an $\mathbf{L}_{0}^{0}$-orbit in the flag manifold $X^{\prime}=\mathbf{L} /\left(\mathbf{Q}^{\prime} \cap \mathbf{L}\right)$.

Next we note that the intersection of $\mathbf{Q}^{\prime}$ with the unipotent subgroup $\mathbf{N}$ is a subgroup of its unipotent radical $\mathbf{Q}^{\prime n}$. Thus it is connected and simply connected and the quotient $Y=\mathbf{N} /\left(\mathbf{Q}^{\prime} \cap \mathbf{N}\right)$ is a connected and simply connected complex nilmanifold. Since both $\mathbf{Q}^{\prime} \cap \mathbf{G}_{0}$ and $\mathbf{Q}^{\prime} \cap \mathbf{N}$ are closed and connected, and we have a Lie algebras semidirect sum decomposition:

$$
\mathfrak{q}^{\prime} \cap \overline{\mathfrak{q}}=\left(\mathfrak{q}^{\prime} \cap \mathfrak{l}\right) \ltimes\left(\mathfrak{q}^{\prime} \cap \mathfrak{n}\right),
$$

we also obtain a semidirect product decomposition:

$$
\mathbf{Q}^{\prime} \cap \overline{\mathbf{Q}}=\left(\mathbf{Q}^{\prime} \cap \mathbf{L}\right) \ltimes\left(\mathbf{Q}^{\prime} \cap \mathbf{N}\right)
$$

Hence the fiber $F=\mathbf{I}_{0} / \mathbf{I}_{0}^{\prime}$ is $C R$ diffeomorphic to the Cartesian product $F=$ $F^{\prime} \times F^{\prime \prime}$, with the $F^{\prime}$ described above, and where $F^{\prime \prime}$ is the orbit of $\mathbf{N}_{0}$ in $Y$. Since $\mathfrak{q}^{\prime} \cap \mathfrak{n} \supset \mathfrak{q}^{\prime n} \cap \mathfrak{q}^{n} \cap \overline{\mathfrak{q}}=\mathfrak{q}^{n} \cap \overline{\mathfrak{q}}$, we have

$$
\mathfrak{n}_{0}+\left(\mathfrak{q}^{\prime} \cap \mathfrak{n}\right) \supset\left(\mathfrak{q}^{\prime} \cap \mathfrak{n}\right)+\left(\overline{\mathfrak{q}^{\prime} \cap \mathfrak{n}}\right) \supset\left(\mathfrak{q}^{n} \cap \overline{\mathfrak{q}}\right)+\left(\overline{\mathfrak{q}}^{n} \cap \mathfrak{q}\right)=\mathfrak{n} .
$$

Thus $\left(\mathfrak{n}_{0}, \mathfrak{q}^{\prime} \cap \mathfrak{n}\right)$ is totally complex. The orbit $F^{\prime \prime}$ of $\mathbf{N}_{0}$ in $Y$, being an open Euclidean complex submanifold of $Y$, coincides with it, because $\mathbf{N}_{0}$ is nilpotent.

Finally, if $\mathfrak{q} \subset \mathfrak{q}^{\prime} \subset \mathfrak{q}+\overline{\mathfrak{q}}$, the factor $F^{\prime}$ in the decomposition (5.12) is an open orbit in $X^{\prime}$ and hence simply connected by [28, Theorem 5.4].

\section{6. $\mathbf{G}_{0}$-equivariant $C R$ fibrations and fit Weyl chambers}

In this section we describe the fundamental and the weakly nondegenerate reductions (see [21]) of a parabolic $C R$ manifold $M$, with associated $C R$ algebra $\left(\mathfrak{g}_{0}, \mathfrak{q}\right)$. This description will be obtained in terms of representations $\mathfrak{q}=\mathfrak{q}_{\Phi}$ of the parabolic subalgebra $\mathfrak{q}$ with respect to systems $\Phi$ of $C$-positive simple roots for $S$-fit and $V$ fit Weyl chambers $C$. Since its fundamental and weakly nondegenerate reductions share with $\left(\mathfrak{g}_{0}, \mathfrak{q}\right)$ the same adapted Cartan pairs, we can fix throughout this section a Cartan pair $\left(\vartheta, \mathfrak{h}_{0}\right)$, adapted to the parabolic $C R$ algebra $\left(\mathfrak{g}_{0}, \mathfrak{q}\right)$. 
Lemma 6.1. Let $\left(\mathfrak{g}_{0}, \mathfrak{q}_{\Phi}\right)$ be a parabolic $C R$ algebra, with $\Phi \subset \mathcal{B}$, where $\mathcal{B}$ is the set of $C$-positive simple roots for an $S$-fit Weyl chamber $C$. Set

$$
\Phi^{-}=\{\alpha \in \Phi \mid \bar{\alpha} \prec 0\} .
$$

Let $\alpha_{0} \in \mathcal{B}$. A necessary and sufficient condition in order that:

$$
\mathfrak{q}_{\Phi}+\overline{\mathfrak{q}}_{\Phi} \subset \mathfrak{q}_{\left\{\alpha_{0}\right\}}
$$

is that

$$
\alpha_{0} \in \Phi \cap \overline{\mathcal{Q}}_{\Phi}^{n} \quad \text { and } \quad \alpha_{0} \notin \bigcup_{\beta \in(\mathcal{B} \backslash \Phi) \cup \Phi^{-}} \operatorname{supp}(\bar{\beta}) .
$$

Proof. First we show that (6.3) implies (6.2). If $\alpha_{0} \in \Phi \cap \overline{\mathcal{Q}}_{\Phi}^{n}$, then $\bar{\alpha}_{0} \succ 0$, because all roots in $\mathcal{Q}_{\Phi}^{n}$ are $C$-positive, and hence, in particular, $\alpha_{0} \notin \mathcal{R}_{\text {im }}$.

We have $\mathfrak{q}_{\Phi} \subset \mathfrak{q}_{\left\{\alpha_{0}\right\}}$, because $\left\{\alpha_{0}\right\} \subset \Phi$. To prove that also $\overline{\mathfrak{q}}_{\Phi} \subset \mathfrak{q}_{\left\{\alpha_{0}\right\}}$, it suffices to show that $\mathfrak{q}_{\left\{\alpha_{0}\right\}}^{n} \subset \overline{\mathfrak{q}}_{\Phi}^{n}$. Assume by contradiction that this inclusion is false. Then there is a root $\alpha$ with $\alpha \succ \alpha_{0}$ and $\alpha \notin \overline{\mathcal{Q}}_{\Phi}^{n}$, i.e. with $\bar{\alpha} \notin \mathcal{Q}_{\Phi}^{n}$. If $\bar{\alpha} \succ 0$, then $\operatorname{supp}(\bar{\alpha}) \cap \Phi=\emptyset$. Being:

$$
\alpha_{0} \in \operatorname{supp}(\alpha) \subset \bigcup_{\beta \in \operatorname{supp}(\bar{\alpha})} \operatorname{supp}(\bar{\beta}),
$$

this would imply that $\alpha_{0} \in \operatorname{supp}(\bar{\beta})$ for some $\beta \in \mathcal{B} \backslash \Phi$. Hence, by $(6.3), \bar{\alpha} \prec 0$, and, from (6.4), we obtain that $\alpha_{0}$ belongs to $\operatorname{supp}(\bar{\beta})$ for some $\beta \in\left(\mathcal{B} \backslash \mathcal{R}_{\text {im }}\right)$ with $\bar{\beta} \prec 0$. But then, because $C$ is $S$-fit, $\beta \in \Phi^{-}$, yielding, by (6.3), a contradiction. This completes the proof that (6.3) implies (6.2).

Let us prove the opposite implication. From $\mathfrak{q}_{\Phi} \subset \mathfrak{q}_{\left\{\alpha_{0}\right\}}$, we have that $\alpha_{0} \in$ $\Phi$. Condition (6.2) is equivalent to the inclusion $\mathfrak{q}_{\{\alpha\}}^{n} \subset \mathfrak{q}_{\Phi}^{n} \cap \overline{\mathfrak{q}}_{\Phi}^{n}$. In particular, $\alpha_{0} \in \overline{\mathcal{Q}}_{\Phi}^{n}$, and, as we already have $\alpha_{0} \in \Phi$, this brings the first half of (6.3). Let $\beta \in \mathcal{B}$ and assume that $\alpha_{0} \in \operatorname{supp}(\bar{\beta})$. If $\bar{\beta} \succ 0$, then $\bar{\beta} \in \mathcal{Q}_{\left\{\alpha_{0}\right\}}^{n} \subset \mathcal{Q}^{n} \cap \overline{\mathcal{Q}}^{n}$ yields $\beta \in \mathcal{Q}^{n}$ and hence, being simple, $\beta \in\left(\Phi \backslash \Phi^{-}\right)$. Otherwise, $\bar{\beta} \prec 0$, and $-\bar{\beta} \in \mathcal{Q}_{\left\{\alpha_{0}\right\}}^{n} \subset \mathcal{Q}_{\Phi}^{n} \cap \overline{\mathcal{Q}}_{\Phi}^{n}$. This would imply that $-\beta \in \mathcal{Q}_{\Phi}^{n}$, but this is impossible because $\mathcal{Q}_{\Phi}^{n}$ consists of $C$-positive roots.

We recall that the basis of the fundamental reduction of a $C R$ algebra $\left(\mathfrak{g}_{0}, \mathfrak{q}\right)$ is the $C R$ algebra $\left(\mathfrak{g}_{0}, \mathfrak{q}^{\prime}\right)$, where $\mathfrak{q}^{\prime}$ is the smallest complex Lie subalgebra of $\mathfrak{g}$ with $\mathfrak{q}+\overline{\mathfrak{q}} \subset \mathfrak{q}^{\prime}$ (see [21, Section 5B]).

Theorem 6.2 (Fundamental reduction). Let $\left(\mathfrak{g}_{0}, \mathfrak{q}_{\Phi}\right)$ be a parabolic CR algebra, with $\Phi \subset \mathcal{B}$, where $\mathcal{B}$ is the set of $C$-positive simple roots for an $S$-fit Weyl chamber C. Let:

$$
\begin{aligned}
\Phi^{-} & =\{\alpha \in \Phi \mid \bar{\alpha} \prec 0\} \\
\Psi & =\Phi \cap \overline{\mathcal{Q}}_{\Phi}^{n} \backslash\left(\bigcup_{\alpha \in(\mathcal{B} \backslash \Phi) \cup \Phi^{-}} \operatorname{supp}(\bar{\alpha})\right) .
\end{aligned}
$$


Then:

(1) $\left(\mathfrak{g}_{0}, \mathfrak{q}_{\Psi}\right)$ is the basis of the fundamental reduction of $\left(\mathfrak{g}_{0}, \mathfrak{q}_{\Phi}\right)$.

(2) A necessary and sufficient condition for $\left(\mathfrak{g}_{0}, \mathfrak{q}_{\Phi}\right)$ to be fundamental is that $\Psi=\emptyset$, i.e. that:

$$
\Phi \cap \overline{\mathcal{Q}}_{\Phi}^{n} \subset \bigcup_{\alpha \in(\mathcal{B} \backslash \Phi) \cup \Phi^{-}} \operatorname{supp}(\bar{\alpha}) .
$$

Let $M_{\Phi}$ and $M_{\Psi}$ be the $\mathbf{G}_{0}$-orbits associated to $\left(\mathfrak{g}_{0}, \mathfrak{q}_{\Phi}\right)$, $\left(\mathfrak{g}_{0}, \mathfrak{q}_{\Psi}\right)$, respectively. Then the $\mathbf{G}_{0}$-equivariant fibration $M_{\Phi} \stackrel{\pi}{\rightarrow} M_{\Psi}$ is $C R$ and all connected components of its fibers are parabolic CR manifolds (cf. Definition 3.1) of finite type. In particular, $M_{\Phi}$ is of finite type if and only if (6.7) holds true.

Proof. The complex subalgebra $\mathfrak{q}^{\prime}$ yielding the fundamental reduction $\left(\mathfrak{g}_{0}, \mathfrak{q}^{\prime}\right)$ of $\left(\mathfrak{g}_{0}, \mathfrak{q}_{\Phi}\right)$ is a complex subalgebra of $\mathfrak{g}$ that contains $\mathfrak{q}_{\Phi}$, and hence is parabolic and of the form $\mathfrak{q}^{\prime}=\mathfrak{q}_{\Phi^{\prime}}$ for some $\Phi^{\prime} \subset \Phi$. Thus $\mathfrak{q}^{\prime}$ is the intersection of all $\mathfrak{q}_{\left\{\alpha_{0}\right\}}$, with $\alpha_{0} \in \Phi$, for which $\mathfrak{q}_{\Phi}+\overline{\mathfrak{q}}_{\Phi} \subset \mathfrak{q}_{\left\{\alpha_{0}\right\}}$. By Lemma 6.1, we have $\Phi^{\prime}=\Psi$. This proves (1) and (2).

The last statement is a consequence of [21, Theorem 5.3].

Next we turn our consideration to weak nondegeneracy. First we prove:

Proposition 6.3. Let $\left(\mathfrak{g}_{0}, \mathfrak{q}_{\Phi}\right)$ and $\left(\mathfrak{g}_{0}, \mathfrak{q}_{\Psi}\right)$ be parabolic $C R$ algebras, with $\Psi \subset$ $\Phi \subset \mathcal{B}$, where $\mathcal{B}$ is the set of $C$-positive simple roots for a $V$-fit Weyl chamber $C$. Let $M_{\Phi}, M_{\Psi}$ be the corresponding $\mathbf{G}_{0}$-orbits. Then the $\mathbf{G}_{0}$-equivariant fibration:

$$
M_{\Phi} \stackrel{\pi}{\longrightarrow} M_{\Psi}
$$

is a CR fibration with complex fibers if and only if:

$$
\bar{\alpha} \prec 0 \quad \forall \alpha \in \Phi \backslash \Psi .
$$

Proof. The necessary and sufficient condition for (6.8) being a $C R$ fibration with complex fiber is that:

$$
\mathfrak{q}_{\Phi} \subset \mathfrak{q}_{\Psi} \subset \mathfrak{q}_{\Phi}+\overline{\mathfrak{q}}_{\Phi}
$$

(see [21, Corollary 5.6]). We need to show that this condition is equivalent to (6.9). It suffices to discuss the situation where $\Phi \backslash \Psi$ consists of a single simple root. So we assume that $\alpha_{0} \in \Phi$ and $\Psi=\Phi \backslash\left\{\alpha_{0}\right\}$.

Assume that (6.10) holds true. Then $-\alpha_{0} \in \overline{\mathcal{Q}}_{\Phi}$, i.e. $-\bar{\alpha}_{0} \in \mathcal{Q}_{\Phi}$. In particular, since $-\alpha_{0} \notin \mathcal{Q}_{\Phi}, \alpha_{0}$ is not real. Thus, if $\bar{\alpha}_{0} \in \mathcal{Q}_{\Phi}^{r}$, then $\bar{\alpha}_{0} \prec 0$, because $C$ is $V$-fit for $\left(\mathfrak{g}_{0}, \mathfrak{q}_{\Phi}\right)$. Otherwise, $-\bar{\alpha}_{0} \in \mathcal{Q}_{\Phi}^{n}$ implies that $\bar{\alpha}_{0} \prec 0$, because $\mathcal{Q}_{\Phi}^{n}$ consists of $C$-positive roots.

Vice versa, let us show that, if $\bar{\alpha}_{0} \prec 0$, then the parabolic set $\mathcal{Q}_{\Psi}$ of $\mathfrak{q} \Psi$ is contained in $\mathcal{Q}_{\Phi} \cup \overline{\mathcal{Q}}_{\Phi}$. This is equivalent to $\mathcal{Q}_{\Psi}^{n} \supset \mathcal{Q}_{\Phi}^{n} \cap \overline{\mathcal{Q}}_{\Phi}^{n}$. Assume by contradiction that there is some $\alpha \in\left(\mathcal{Q}_{\Phi}^{n} \cap \overline{\mathcal{Q}}_{\Phi}^{n}\right) \backslash \mathcal{Q}_{\Psi}^{n}$. Then $\operatorname{supp}(\alpha) \cap \Phi=\left\{\alpha_{0}\right\}$, but this yields $\bar{\alpha} \prec 0$. Indeed, $\bar{\beta} \prec 0$ for all non real roots $\beta$ in the support of $\alpha$, because $C$ is $V$-fit for $\left(\mathfrak{g}_{0}, \mathfrak{q}_{\Phi}\right)$, and we assumed that $\bar{\alpha}_{0} \prec 0$. This gives a contradiction, since $\bar{\alpha} \in \mathcal{Q}_{\Phi}^{n}$, that consists of $C$-positive roots. 
We recall (see [21, Lemma 5.7]) that, given any $C R$ algebra $\left(\mathfrak{g}_{0}, \mathfrak{q}\right)$, there is a unique maximal complex subalgebra $\mathfrak{q}^{\prime}$ of $\mathfrak{g}$ with $\mathfrak{q} \subset \mathfrak{q}^{\prime} \subset(\mathfrak{q}+\overline{\mathfrak{q}})$. The $C R$ algebra $\left(\mathfrak{g}_{0}, \mathfrak{q}^{\prime}\right)$ is (see $[21$, Section $\left.5 C]\right)$ the basis of the weakly nondegenerate reduction of $\left(\mathfrak{g}_{0}, \mathfrak{q}\right)$.

We obtain:

Theorem 6.4 (Weakly nondegenerate reduction). Let $\left(\mathfrak{g}_{0}, \mathfrak{q}_{\Phi}\right)$ be a parabolic $C R$ algebra, with $\Phi \subset \mathcal{B}$, where $\mathcal{B}$ is the set of $C$-positive simple roots for a $V$-fit Weyl chamber C. Set:

$$
\Psi=\{\alpha \in \Phi \mid \bar{\alpha} \succ 0\} .
$$

Then the parabolic CR algebra $\left(\mathfrak{g}_{0}, \mathfrak{q}_{\Psi}\right)$ is the basis of the weakly nondegenerate reduction of $\left(\mathfrak{g}_{0}, \mathfrak{q}_{\Phi}\right)$.

Let $M_{\Phi}, M_{\Psi}$ be the $\mathbf{G}_{0}$-orbits corresponding to $\left(\mathfrak{g}_{0}, \mathfrak{q}_{\Phi}\right)$, $\left(\mathfrak{g}_{0}, \mathfrak{q}_{\Psi}\right)$, respectively. Then the $\mathbf{G}_{0}$-orbit $M_{\Psi}$ is holomorphically nondegenerate and the $\mathbf{G}_{0}$-equivariant fibration $M_{\Phi} \stackrel{\pi}{\rightarrow} M_{\Psi}$ is a CR fibration with simply connected (but not necessarily connected) complex fibers.

In particular, $M_{\Phi}$ is holomorphically nondegenerate if and only if:

$$
\bar{\alpha} \succ 0 \quad \forall \alpha \in \Phi
$$

Proof. All complex subalgebras $\mathfrak{q}^{\prime}$ containing $\mathfrak{q}_{\Phi}$ are parabolic and of the form $\mathfrak{q}^{\prime}=\mathfrak{q}_{\Psi}$ for some $\Psi \subset \Phi$. Thus, by Proposition 6.3, condition (6.12) is necessary and sufficient for $M_{\Phi}$ to be holomorphically nondegenerate.

In general, we observe that, if $C$ is $V$-fit for $\left(\mathfrak{g}_{0}, \mathfrak{q}\right)$, with $\mathfrak{q}=\mathfrak{q}_{\Phi}$, and $\Psi$ is defined by (6.11), then the chamber $C$ is $V$-fit also for $\left(\mathfrak{g}_{0}, \mathfrak{q}_{\Psi}\right)$, and therefore $M_{\Psi}$ is holomorphically nondegenerate by the argument above. Proposition 6.3 tells us that $M_{\Phi} \stackrel{\pi}{\rightarrow} M_{\Psi}$ is a $C R$ fibration with a holomorphically nondegenerate basis and a complex fiber. Then the statement follows by the uniqueness of the weakly nondegenerate reduction (see [21, Lemma 5.7]) and from the last statement in Theorem 5.10 .

\section{A structure theorem for $\mathbf{G}_{0}$-orbits}

Let $M$ be any $\mathbf{G}_{0}$-orbit, with associated parabolic $C R$ algebra $\left(\mathfrak{g}_{0}, \mathfrak{q}\right)$. It is easy to construct a smooth $\mathbf{G}_{0}$-equivariant fibration, with complex fibers, of $M$ onto a real flag manifold $M^{\prime}$ of $\mathbf{G}_{0}$. Fix indeed a Cartan pair $\left(\vartheta, \mathfrak{h}_{0}\right)$ adapted to $\left(\mathfrak{g}_{0}, \mathfrak{q}\right)$, and let $\mathcal{R}$ be the roots of $\mathfrak{g}$ with respect to the complexification $\mathfrak{h}$ of $\mathfrak{h}_{0}$. Choose any element $A$ of the facet in $\mathfrak{h}_{\mathbb{R}}$ corresponding to $\mathfrak{q}(c f$. [8, VIII Section 4]). Then the facet of $B=A+\bar{A}$ defines a parabolic $\mathfrak{q}^{\prime}$ with $\mathfrak{q} \cap \overline{\mathfrak{q}} \subset \mathfrak{q}^{\prime}=\overline{\mathfrak{q}}^{\prime} \subset(\mathfrak{q}+\overline{\mathfrak{q}})$. The $\mathbf{G}_{0^{-}}$ equivariant fibration $M \rightarrow M^{\prime}$ of $M$ onto the parabolic $C R$ manifold corresponding to $\left(\mathfrak{g}_{0}, \mathfrak{q}^{\prime}\right)$ has all the required properties. A different canonical construction is given by Wolf ( $c f$. Section 11). The construction below is also canonical, and allows a better control of the structure of the complex fibers. 
Starting from a parabolic $C R$ algebra $\left(\mathfrak{g}_{0}, \mathfrak{q}\right)$, we construct recursively a sequence of parabolic $C R$ algebras $\left(\mathfrak{g}_{0}, \mathfrak{q}^{(h)}\right)$ by setting, for all $h \geq 0$ :

$$
\left\{\begin{array}{l}
\left(\mathfrak{g}_{0}, \mathfrak{q}^{(0)}\right)=\text { the weakly nondegenerate reduction of }\left(\mathfrak{g}_{0}, \mathfrak{q}\right) \\
\left(\mathfrak{g}_{0}, \mathfrak{q}^{(h+1)}\right)=\text { the weakly nondegenerate reduction of }\left(\mathfrak{g}_{0}, \mathfrak{q}_{w}^{(h)}\right)
\end{array}\right.
$$

We recall that $\left(\mathfrak{g}_{0}, \mathfrak{q}_{w}^{(h)}\right)$ is the $C R$-weakening of $\left(\mathfrak{g}_{0}, \mathfrak{q}^{(h)}\right)$, defined in Theorem 5.3. The weakly nondegenerate reduction was described in Theorem 6.4. Denote by $M^{(h)}$ and by $M_{w}^{(h)}$ the $\mathbf{G}_{0}$-orbits associated to the $C R$ algebras $\left(\mathfrak{g}_{0}, \mathfrak{q}^{(h)}\right)$ and $\left(\mathfrak{g}_{0}, \mathfrak{q}_{w}^{(h)}\right)$, respectively. We know that there is a $\mathbf{G}_{0}$-equivariant diffeomorphism $f_{h}: M_{w}^{(h)} \rightarrow$ $M^{(h)}$, which is also a $C R$ map, but in general not a $C R$ diffeomorphism. Let $\psi_{h}: M^{(h)} \rightarrow M^{(h+1)}$ the composition of the weakly nondegenerate reduction $\pi_{h}: M_{w}^{(h)} \rightarrow M^{(h+1)}$ and of the inverse of $f_{h}$. We have a commutative diagram:

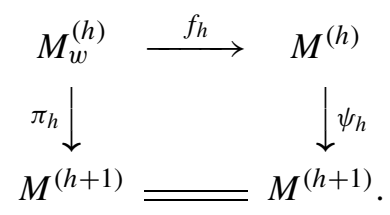

Denoting by $\pi: M \rightarrow M^{(0)}$ the weakly nondegenerate reduction, we obtain a sequence of smooth $\mathbf{G}_{0}$-equivariant maps:

$$
M \stackrel{\pi}{\longrightarrow} M^{(0)} \stackrel{\psi_{0}}{\longrightarrow} M^{(1)} \stackrel{\psi_{1}}{\longrightarrow} M^{(2)} \longrightarrow \cdots
$$

In general, the maps $\psi_{h}$ are not $C R$. Since

$$
\operatorname{dim} M^{(h)} \leq \operatorname{dim} M^{(h+1)} \quad \forall h \geq 0,
$$

and, by Proposition 5.7, equality in (7.3) holds if and only if $\left(\mathfrak{g}_{0}, \mathfrak{q}_{w}^{(h)}\right)$ is totally real, the sequence $\left\{\mathfrak{q}^{(h)}\right\}$ stabilizes and we have, for some integer $m \geq 0$,

$$
\mathfrak{q}^{(h)}=\mathfrak{q}_{w}^{(h)}=\mathfrak{q}^{(m)}=\mathfrak{e}, \quad \forall h \geq m .
$$

Definition 7.1. The limit manifold $M_{\mathfrak{e}}$ of (7.2), which is the $\mathbf{G}_{0}$-orbit associated to the $C R$ algebra $\left(\mathfrak{g}_{0}, \mathfrak{e}\right)$, is called the real core of $M$.

Theorem 7.2 (Structure theorem). Let $\left(\mathfrak{g}_{0}, \mathfrak{q}\right)$ be a parabolic CR algebra, $M$ the corresponding $\mathbf{G}_{0}$-orbit, and $M_{\mathfrak{e}}$, with $C R$ algebra $\left(\mathfrak{g}_{0}, \mathfrak{e}\right)$, its real core. Then:

(1) $\left(\mathfrak{g}_{0}, \mathfrak{e}\right)$ is totally real, i.e. $\overline{\mathfrak{e}}=\mathfrak{e}$;

(2) $M_{\mathfrak{e}}$ is a real flag manifold and the $\mathbf{G}_{0}$-equivariant projection:

$$
M \stackrel{\psi}{\longrightarrow} M_{\mathfrak{e}}
$$

has complex fibers, with finitely many connected components, that are simply connected; 
(3) each connected component $F_{0}$ of a fiber $F$ of (7.5) is a tower of holomorphic fibrations, in which each fiber is the Cartesian product of a Euclidean complex space and an open orbit of a real form in a complex flag manifold.

Proof. We already established (1) and the first statement of (2). The weakly nondegenerate reduction $M_{w}^{(h)} \rightarrow M^{(h+1)}$ is a $C R$ fibration for which we know, by Theorem 5.10 and Theorem 6.4, that the fiber has finitely many components and each connected component is a Cartesian product of a Euclidean complex space and an open orbit of a real form in a complex flag manifold, hence we have also (2) and (3).

\section{Connected components of the fibers}

We keep the notation of Section 3. Let $\left(\vartheta, \mathfrak{h}_{0}\right)$ be a Cartan pair, adapted to the parabolic $C R$ algebra $\left(\mathfrak{g}_{0}, \mathfrak{q}\right)$ of the $\mathbf{G}_{0}$-orbit $M$. Denote by $\mathbf{H}_{0}=\mathbf{Z}_{\mathbf{G}_{0}}\left(\mathfrak{h}_{0}\right)$ the Cartan subgroup of $\mathbf{G}_{0}$ corresponding to the Cartan subalgebra $\mathfrak{h}_{0}$.

Definition 8.1. The analytic, or real, Weyl group $\mathbf{W}\left(\mathbf{G}_{0}, \mathfrak{h}_{0}\right)$ is the quotient by $\mathbf{H}_{0}$ of the normalizer of $\mathfrak{h}_{0}$ in $\mathbf{G}_{0}$ :

$$
\mathbf{W}\left(\mathbf{G}_{0}, \mathfrak{h}_{0}\right)=\mathbf{N}_{\mathbf{G}_{0}}\left(\mathfrak{h}_{0}\right) / \mathbf{Z}_{\mathbf{G}_{0}}\left(\mathfrak{h}_{0}\right) .
$$

Since we took $\mathbf{G}_{0}$ connected, the analytic Weyl group $\mathbf{W}\left(\mathbf{G}_{0}, \mathfrak{h}_{0}\right)$ only depends, modulo isomorphisms, upon the pair $\left(\mathfrak{g}_{0}, \mathfrak{h}_{0}\right)$, and not on the particular choice of $\mathbf{G}_{0}$. We also have (see e.g. [16, page 489])

$$
\mathbf{W}\left(\mathbf{G}_{0}, \mathfrak{h}_{0}\right)=\mathbf{N}_{\mathbf{K}_{0}}\left(\mathfrak{h}_{0}\right) / \mathbf{Z}_{\mathbf{K}_{0}}\left(\mathfrak{h}_{0}\right),
$$

where $\mathbf{K}_{0}$ is the compact analytic subgroup of $\mathbf{G}_{0}$ with Lie algebra $\mathfrak{k}_{0}=\left\{X \in \mathfrak{g}_{0} \mid\right.$ $\vartheta(X)=X\}$. Since, by complexification, $\mathbf{N}_{\mathbf{G}_{0}}\left(\mathfrak{h}_{0}\right)$ acts on $\mathfrak{h}_{\mathbb{R}}=\mathfrak{h}_{0}^{-} \oplus i \mathfrak{h}_{0}^{+}$, and then, by duality, on $\mathfrak{h}_{\mathbb{R}}^{*}$ and, by restriction, on the root system $\mathcal{R}=\mathcal{R}(\mathfrak{g}, \mathfrak{h})$, we obtain a homomorphism, that in fact is an inclusion:

$$
\mathbf{W}\left(\mathbf{G}_{0}, \mathfrak{h}_{0}\right) \hookrightarrow \mathbf{W}(\mathcal{R}),
$$

of the analytic Weyl group $\mathbf{W}\left(\mathbf{G}_{0}, \mathfrak{h}_{0}\right)$ into the Weyl group $\mathbf{W}(\mathcal{R})$ of the root system $\mathcal{R}$.

We shall also consider the Weyl group:

$$
\mathbf{W}\left(\mathbf{L}_{0}, \mathfrak{h}_{0}\right)=\mathbf{N}_{\mathbf{L}_{0}}\left(\mathfrak{h}_{0}\right) / \mathbf{Z}_{\mathbf{L}_{0}}\left(\mathfrak{h}_{0}\right)
$$

of the reductive subgroup $\mathbf{L}_{0}$. Since $\mathbf{Z}_{\mathbf{L}_{0}}\left(\mathfrak{h}_{0}\right)=\mathbf{H}_{0}$, this can be viewed as a subgroup of the analytic Weyl group $\mathbf{W}\left(\mathbf{G}_{0}, \mathfrak{h}_{0}\right)$.

Let $\mathbf{S}_{0}$ be the analytic semisimple subgroup of $\mathbf{L}_{0}$ with Lie algebra $\mathfrak{s}_{0}=\left[\mathfrak{l}_{0}, \mathfrak{l}_{0}\right]$ (see Corollary 3.5). The elements of $\mathbf{S}_{0}$ centralize $\mathfrak{z}_{0}$. Thus, for the Cartan subalgebra $\mathfrak{t}_{0}=\mathfrak{h}_{0} \cap \mathfrak{s}_{0}$ of $\mathfrak{s}_{0}$, since $\mathfrak{h}_{0}=\mathfrak{t}_{0} \oplus \mathfrak{z}_{0}$, we get:

$$
\mathbf{N}_{\mathbf{S}_{0}}\left(\mathfrak{t}_{0}\right)=\mathbf{N}_{\mathbf{S}_{0}}\left(\mathfrak{h}_{0}\right) \subset \mathbf{N}_{\mathbf{L}_{0}}\left(\mathfrak{h}_{0}\right) \text {. }
$$


The Cartan subgroup $\mathbf{T}_{0}$ of $\mathbf{S}_{0}$ corresponding to $\mathfrak{t}_{0}$ is:

$$
\mathbf{T}_{0}=\mathbf{Z}_{\mathbf{S}_{0}}\left(\mathfrak{t}_{0}\right)=\mathbf{Z}_{\mathbf{S}_{0}}\left(\mathfrak{h}_{0}\right)=\mathbf{H}_{0} \cap \mathbf{S}_{0}
$$

Thus the inclusion (8.5) yields, by passing to the quotients, an inclusion of the Weyl groups:

$$
\mathbf{W}\left(\mathbf{S}_{0}, \mathfrak{t}_{0}\right)=\frac{\mathbf{N}_{\mathbf{S}_{0}}\left(\mathfrak{t}_{0}\right)}{\mathbf{Z}_{\mathbf{S}_{0}}\left(\mathfrak{t}_{0}\right)} \simeq \frac{\mathbf{N}_{\mathbf{S}_{0}}\left(\mathfrak{t}_{0}\right) \mathbf{Z}_{\mathbf{L}_{0}}\left(\mathfrak{h}_{0}\right)}{\mathbf{Z}_{\mathbf{L}_{0}}\left(\mathfrak{h}_{0}\right)} \subset \mathbf{W}\left(\mathbf{L}_{0}, \mathfrak{h}_{0}\right) .
$$

Proposition 8.2. Let Mbe a $\mathbf{G}_{0}$-orbit, with associated parabolic CR algebra $\left(\mathfrak{g}_{0}, \mathfrak{q}\right)$. Let $\left(\vartheta, \mathfrak{h}_{0}\right)$ be a Cartan pair adapted to $\left(\mathfrak{g}_{0}, \mathfrak{q}\right), \mathbf{L}_{0}$ the $\vartheta$-invariant reductive Levi factor of the isotropy $\mathbf{I}_{0}$ of $M$, and $\mathbf{S}_{0}$ the maximal analytic semisimple Lie subgroup of $\mathbf{L}_{0}$. Then:

(1) $\mathbf{N}_{\mathbf{S}_{0}}\left(\mathfrak{h}_{0}\right)$ is a closed normal subgroup of $\mathbf{N}_{\mathbf{L}_{0}}\left(\mathfrak{h}_{0}\right)$ and the natural inclusion $\mathbf{N}_{\mathbf{L}_{0}}\left(\mathfrak{h}_{0}\right) \hookrightarrow \mathbf{I}_{0}$ yields a group isomorphism:

$$
\pi_{0}\left(\mathbf{N}_{\mathbf{L}_{0}}\left(\mathfrak{h}_{0}\right) / \mathbf{N}_{\mathbf{S}_{0}}\left(\mathfrak{h}_{0}\right)\right) \stackrel{\sim}{\longrightarrow} \pi_{0}\left(\mathbf{I}_{0}\right) .
$$

(2) We have the exact sequences:

$$
\begin{gathered}
\mathbf{1} \longrightarrow \frac{\mathbf{H}_{0}}{\mathbf{T}_{0}} \longrightarrow \frac{\mathbf{N}_{\mathbf{L}_{0}}\left(\mathfrak{h}_{0}\right)}{\mathbf{N}_{\mathbf{S}_{0}}\left(\mathfrak{t}_{0}\right)} \longrightarrow \frac{\mathbf{W}\left(\mathbf{L}_{0}, \mathfrak{h}_{0}\right)}{\mathbf{W}\left(\mathbf{S}_{0}, \mathfrak{t}_{0}\right)} \longrightarrow \mathbf{1}, \\
\mathbf{1} \longrightarrow \pi_{0}\left(\frac{\mathbf{H}_{0}}{\mathbf{T}_{0}}\right) \longrightarrow \frac{\mathbf{W}\left(\mathbf{L}_{0}, \mathfrak{h}_{0}\right)}{\mathbf{W}\left(\mathbf{S}_{0}, \mathfrak{t}_{0}\right)} \longrightarrow \mathbf{1}
\end{gathered}
$$

Proof. By (3.4), we have $\pi_{0}\left(\mathbf{L}_{0}\right) \simeq \pi_{0}\left(\mathbf{I}_{0}\right)$.

Let $\mathfrak{g}_{0}=\mathfrak{k}_{0} \oplus \mathfrak{p}_{0}$ be the $\vartheta$-invariant Cartan decomposition of $\mathfrak{g}_{0}$, and let $\mathfrak{a}_{0}$ be any maximal Abelian subalgebra of $\mathfrak{p}_{0}$ that contains $\mathfrak{h}_{0}^{-}=\mathfrak{h}_{0} \cap \mathfrak{p}_{0}$. Its centralizer $\mathbf{Z}_{\mathbf{K}_{00}}\left(\mathfrak{a}_{0}\right)$ in the maximal compact subgroup $\mathbf{K}_{00}$ of $\mathbf{L}_{0}$ intersects all connected components of $\mathbf{L}_{0}$ (see e.g. [16, Proposition 7.33]). Thus a fortiori $\mathbf{Z}_{\mathbf{K}_{00}}\left(\mathfrak{h}_{0}^{-}\right)$, containing $\mathbf{Z}_{\mathbf{K}_{00}}\left(\mathfrak{a}_{0}\right)$, intersects all connected components of $\mathbf{L}_{0}$. The toroidal part $\mathfrak{h}_{0}^{+}=\mathfrak{h}_{0} \cap \mathfrak{k}_{0}$ of $\mathfrak{h}_{0}$ is a Cartan subalgebra of the compact Lie algebra $\mathbf{Z}_{\mathfrak{k}_{00}}\left(\mathfrak{h}_{0}^{-}\right)$. Let $g_{0} \in \mathbf{Z}_{\mathbf{K}_{00}}\left(\mathfrak{h}_{0}^{-}\right)$. Then $\operatorname{Ad}\left(g_{0}\right)\left(\mathfrak{h}_{0}^{+}\right)$is also a Cartan subalgebra of $\mathbf{Z}_{\mathfrak{k}_{00}}\left(\mathfrak{h}_{0}^{-}\right)$. Since all Cartan subalgebras of a compact Lie algebra are conjugate by an inner automorphism, we can find an element $g_{1}$, in the connected component $\mathbf{K}_{00}^{0}$ of the identity of $\mathbf{K}_{00}$, such that $\operatorname{Ad}\left(g_{1} g_{0}\right)\left(\mathfrak{h}_{0}^{+}\right)=\mathfrak{h}_{0}^{+}$. The element $g_{1} g_{0} \in \mathbf{Z}_{\mathbf{L}_{0}}\left(\mathfrak{h}_{0}^{-}\right) \cap \mathbf{N}_{\mathbf{L}_{0}}\left(\mathfrak{h}_{0}^{+}\right)$is in the same connected component of $g_{0}$ in $\mathbf{K}_{00}$. This shows that $\mathbf{Z}_{\mathbf{L}_{0}}\left(\mathfrak{h}_{0}^{-}\right) \cap \mathbf{N}_{\mathbf{L}_{0}}\left(\mathfrak{h}_{0}^{+}\right)$, and therefore also $\mathbf{N}_{\mathbf{L}_{0}}\left(\mathfrak{h}_{0}\right)$, intersect all connected components of $\mathbf{L}_{0}$.

Let $\mathbf{Z}_{0}^{0}$ be the analytic subgroup of $\mathbf{L}_{0}$ with Lie algebra $\mathfrak{z}_{0}$. Then the connected component $\mathbf{L}_{0}^{0}$ of the identity in $\mathbf{L}_{0}$ is the direct product $\mathbf{S}_{0} \bowtie \mathbf{Z}_{0}^{0}$. It follows that the intersection $\mathbf{N}_{\mathbf{L}_{0}}\left(\mathfrak{h}_{0}\right) \cap \mathbf{L}_{0}^{0}$ is $\mathbf{N}_{\mathbf{S}_{0}}\left(\mathfrak{h}_{0}\right) \bowtie \mathbf{Z}_{0}^{0}$, and this yields (8.8).

The exactness of (8.10) is a consequence of that of (8.9) and of the isomorphism (8.8). It will suffice then to prove the exactness of (8.9). 
By the definition of the Weyl group $\mathbf{W}\left(\mathbf{L}_{0}, \mathfrak{h}_{0}\right)$, we have an exact sequence:

$$
\mathbf{1} \longrightarrow \mathbf{H}_{0} \longrightarrow \mathbf{N}_{\mathbf{L}_{0}}\left(\mathfrak{h}_{0}\right) \longrightarrow \mathbf{W}\left(\mathbf{L}_{0}, \mathfrak{h}_{0}\right) \longrightarrow 1
$$

The image in $\mathbf{W}\left(\mathbf{L}_{0}, \mathfrak{h}_{0}\right)$ of the subgroup $\mathbf{N}_{\mathbf{S}_{0}}\left(\mathfrak{t}_{0}\right)$ of $\mathbf{N}_{\mathbf{L}_{0}}\left(\mathfrak{h}_{0}\right)$, under the projection into the quotient, using the identification (8.7), is $\mathbf{W}\left(\mathbf{S}_{0}, \mathfrak{t}_{0}\right)$. Finally, the Cartan subgroup $\mathbf{T}_{0}$ of $\mathbf{S}_{0}$ is the intersection $\mathbf{H}_{0} \cap \mathbf{N}_{\mathbf{S}_{0}}\left(\mathfrak{h}_{0}\right)$. Then (8.9) follows from (8.11) by the elementary group homomorphism theorems.

Remark 8.3. Note that the number of connected components of the isotropy subgroup $\mathbf{I}_{0}$ depends on the choice of $\mathbf{G}_{0}$. The exact sequence (8.10) exhibits this number as a product of a term, $\left|\pi_{0}\left(\mathbf{H}_{0} / \mathbf{T}_{0}\right)\right|$, that genuinely depends on $\mathbf{G}_{0}$, and another term, $\left|\left(\mathbf{W}\left(\mathbf{L}_{0}, \mathfrak{h}_{0}\right) / \mathbf{W}\left(\mathbf{S}_{0}, \mathfrak{t}_{0}\right)\right)\right|$, which is the same for all possible choices of the connected linear Lie group $\mathbf{G}_{0}$ with Lie algebra $\mathfrak{g}_{0}$. Indeed, modulo isomorphisms, the groups $\mathbf{W}\left(\mathbf{L}_{0}, \mathfrak{h}_{0}\right)$ and $\mathbf{W}\left(\mathbf{S}_{0}, \mathfrak{t}_{0}\right)$ only depend on the Lie algebras $\mathfrak{g}_{0}$, $\mathfrak{l}_{0}$ and $\mathfrak{h}_{0}$ (see e.g. [16, page 489]).

Our main application of Proposition 8.2 is counting the number of connected components of the fibers of a $\mathbf{G}_{0}$-equivariant fibration (5.2). We have:

Theorem 8.4. Let $M, M^{\prime}$ be $\mathbf{G}_{0}$-orbits, with associated parabolic CR algebras $\left(\mathfrak{g}_{0}, \mathfrak{q}\right),\left(\mathfrak{g}_{0}, \mathfrak{q}^{\prime}\right)$, and isotropy subalgebras $\mathfrak{i}_{0} \supset \mathfrak{i}_{0}^{\prime}$, respectively. Let $\left(\vartheta, \mathfrak{h}_{0}\right)$ be a Cartan pair adapted to $\left(\mathfrak{g}_{0}, \mathfrak{q}^{\prime}\right)$, with $\mathfrak{h}_{0}$ a maximally noncompact Cartan subalgebra of $\mathfrak{i}_{0}^{\prime}$. Then the group of connected components of the typical fiber $F$ of the $\mathbf{G}_{0}$-equivariant fibration $\phi: M^{\prime} \rightarrow M$ is

$$
\pi_{0}(F) \simeq \mathbf{W}\left(\mathbf{L}_{0}, \mathfrak{h}_{0}\right) / \mathbf{W}\left(\mathbf{S}_{0}, \mathfrak{t}_{0}\right)
$$

where $\mathbf{L}_{0}$ is the reductive $\vartheta$-invariant Levi factor of $\mathbf{I}_{0}$, having maximal semisimple analytic subgroup $\mathbf{S}_{0}$, with Lie algebra $\mathfrak{s}_{0}$, and $\mathfrak{t}_{0}=\mathfrak{h}_{0} \cap \mathfrak{s}_{0}$.

Proof. Since $\mathfrak{h}_{0}$ is a maximally noncompact Cartan subalgebra of $\mathfrak{i}_{0}^{\prime}$, by Theorem 3.6 we have the isomorphism $\pi_{0}\left(\mathbf{H}_{0} / \mathbf{T}_{0}^{\prime}\right) \simeq \pi_{0}\left(\mathbf{I}_{0}^{\prime}\right)$, where $\mathbf{T}_{0}^{\prime}$ is the centralizer of $\mathbf{H}_{0}$ in $\mathbf{S}_{0}^{\prime}$, yielding a commutative diagram

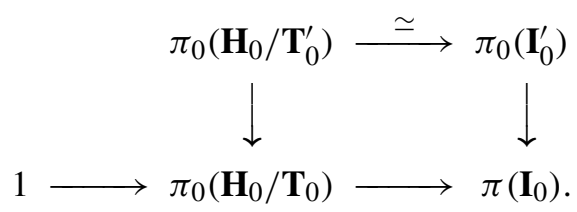

Since the map $\pi_{0}\left(\mathbf{H}_{0} / \mathbf{T}_{0}^{\prime}\right) \rightarrow \pi_{0}\left(\mathbf{H}_{0} / \mathbf{T}_{0}\right)$ is surjective, from (8.10) we obtain the exact sequence:

$$
\pi_{0}\left(\mathbf{I}_{0}^{\prime}\right) \longrightarrow \pi_{0}\left(\mathbf{I}_{0}\right) \longrightarrow \frac{\mathbf{W}\left(\mathbf{L}_{0}, \mathfrak{h}_{0}\right)}{\mathbf{W}\left(\mathbf{S}_{0}, \mathfrak{t}_{0}\right)} \longrightarrow 1
$$

yielding (8.12). 


\section{The fundamental group}

We use Theorem 7.2 and the results of Section 8 to compute the fundamental group of the $\mathbf{G}_{0}$-orbits, extending the results of [2, Section 8]. We have:

Theorem 9.1. Let $M$ be a $\mathbf{G}_{0}$-orbit, $\left(\mathfrak{g}_{0}, \mathfrak{q}\right)$ its associated parabolic CR algebra, and $M_{\mathfrak{e}}$ its real core (see Definition 7.1), with CR algebra $\left(\mathfrak{g}_{0}, \mathfrak{e}\right)$.

Let $\left(\vartheta, \mathfrak{h}_{0}\right)$ be a Cartan pair adapted to $\left(\mathfrak{g}_{0}, \mathfrak{q}\right)$, with $\mathfrak{h}_{0}$ maximally noncompact in $\mathfrak{i}_{0}$. Set $\mathbf{L}_{0}^{\mathfrak{e}}, \mathbf{S}_{0}^{\mathfrak{e}}$ for the reductive Levi factor of the isotropy $\mathbf{I}_{0}^{\mathfrak{e}}$ of $M_{\mathfrak{e}}$ and for its maximal analytic semisimple subgroup, respectively.

Then we have an exact sequence:

$$
1 \longrightarrow \pi_{1}(M) \longrightarrow \pi_{1}\left(M_{\mathfrak{e}}\right) \longrightarrow \frac{\mathbf{W}\left(\mathbf{L}_{0}^{\mathfrak{e}}, \mathfrak{h}_{0}\right)}{\mathbf{W}\left(\mathbf{S}_{0}^{,}, \mathfrak{h}_{0} \cap \mathfrak{s}_{0}^{e}\right)} \longrightarrow 1
$$

In particular, the image of the map $\pi_{1}(M) \rightarrow \pi_{1}\left(M_{\mathfrak{e}}\right)$ in (9.1) is a normal subgroup of $\pi_{1}\left(M_{\mathfrak{e}}\right)$, with a finite index.

The map $\mathrm{H}_{1}(M, \mathbb{Q}) \rightarrow \mathrm{H}_{1}\left(M_{\mathfrak{e}}, \mathbb{Q}\right)$ of the rational homologies, induced by the $\mathbf{G}_{0}$-equivariant projection $M \rightarrow M_{\mathfrak{e}}$, is an isomorphism.

Proof. By (3) of Theorem 7.2, the fundamental group of the typical fiber $F$ of (7.5) is trivial. Thus, since $M$ is connected, the exact homotopy sequence of a locally trivial fiber bundle yields the short exact sequence:

$$
1 \longrightarrow \pi_{1}(M) \longrightarrow \pi_{1}\left(M_{\mathfrak{e}}\right) \longrightarrow \pi_{0}(F) \longrightarrow 1 .
$$

The exactness of (9.1) follows then from the isomorphism (8.12) established in Theorem 8.4.

Since $F=\mathbf{I}_{0}^{\mathfrak{e}} / \mathbf{I}_{0}$, we have the exact sequence:

$$
\pi_{0}\left(\mathbf{I}_{0}\right) \longrightarrow \pi_{0}\left(\mathbf{I}_{0}^{\mathfrak{e}}\right) \longrightarrow \pi_{0}(F) \longrightarrow 1 .
$$

By Theorem 3.6, the groups $\pi_{0}\left(\mathbf{I}_{0}\right)$ and $\pi_{0}\left(\mathbf{I}_{0}^{\mathfrak{e}}\right)$ are Abelian. Hence $\pi_{0}(F)$, being in a one-to-one correspondence with a quotient of finite Abelian groups, may be given the structure of a finite Abelian group, for which the maps $\pi_{0}\left(\mathbf{I}_{0}^{\mathfrak{e}}\right) \rightarrow \pi_{0}(F)$ and $\pi_{1}\left(M_{\mathfrak{e}}\right) \rightarrow \pi_{0}(F)$ are group epimorphisms. Thus the image of $\pi_{1}(M) \rightarrow \pi_{1}\left(M_{\mathfrak{e}}\right)$ in (9.1) is a normal subgroup of $\pi_{1}\left(M_{\mathfrak{e}}\right)$, being the kernel of a group homomorphism. The last assertion follows from the fact that the kernel of the homomorphism $\pi_{1}(M) \rightarrow \pi_{1}\left(M_{\mathfrak{e}}\right)$ is a torsion subgroup.

Remark 9.2. We see from (9.1) that the fundamental group $\pi_{1}(M)$ only depends on the totally real parabolic $C R$ algebra $\left(\mathfrak{g}_{0}, \mathfrak{e}\right)$ and from the maximally noncompact Cartan subalgebra $\mathfrak{h}_{0}$ of $\mathfrak{i}_{0}$.

We show by the following proposition that, vice versa, for each real flag manifold $M_{\mathfrak{e}}$ of $\mathbf{G}_{0}$, and every Cartan pair $\left(\vartheta, \mathfrak{h}_{0}\right)$ adapted to $\left(\mathfrak{g}_{0}, \mathfrak{e}\right)$, we can find a $\mathbf{G}_{0}$-orbit whose fundamental group is given by (9.1). We have indeed: 
Proposition 9.3. Let $\left(\vartheta, \mathfrak{h}_{0}\right)$ be an adapted Cartan pair for a totally real parabolic CR algebra $\left(\mathfrak{g}_{0}, \mathfrak{y}\right)$. Then we can find a parabolic subalgebra $\mathfrak{q}$ such that:

$\mathfrak{q} \subset \mathfrak{y}=\mathfrak{q}+\overline{\mathfrak{q}}$,

$\mathfrak{h}_{0}$ is a maximally noncompact Cartan subalgebra of $\mathfrak{i}_{0}=\mathfrak{q} \cap \mathfrak{g}_{0}$.

Denote by $M$ the $\mathbf{G}_{0}$-orbit with $C R$ algebra $\left(\mathfrak{g}_{0}, \mathfrak{q}\right)$. Then we have the exact sequence

$$
\mathbf{1} \longrightarrow \pi_{1}(M) \longrightarrow \pi_{1}\left(M_{\mathfrak{y}}\right) \longrightarrow \frac{\mathbf{W}\left(\mathbf{L}_{0}^{\mathfrak{y}}, \mathfrak{h}_{0}\right)}{\mathbf{W}\left(\mathbf{S}_{0}^{\mathfrak{y}}, \mathfrak{h}_{0} \cap \mathfrak{s}_{0}^{\mathfrak{y}}\right)} \longrightarrow \mathbf{1},
$$

where $M_{\mathfrak{y}}$, the $\mathbf{G}_{0}$-orbit associated to $\left(\mathfrak{g}_{0}, \mathfrak{y}\right)$, is a real flag manifold, with isotropy subgroup $\mathbf{I}_{0}^{\mathfrak{y}}, \mathbf{L}_{0}^{\mathfrak{y}}$ is the reductive part of $\mathbf{I}_{0}^{\mathfrak{y}}$, and $\mathbf{S}_{0}^{\mathfrak{y}}$ the maximal analytic semisimple subgroup of $\mathbf{L}_{0}^{\mathfrak{y}}$, with Lie algebra $\mathfrak{s}_{0}^{\mathfrak{y}}$.

Note that $M_{\mathfrak{y}}$ does not necessarily coincide with the real core $M_{\mathfrak{e}}$ of $M$.

Proof. Let $\mathcal{R}$ be the root system of $\mathfrak{g}$ with respect to the complexification $\mathfrak{h}$ of $\mathfrak{h}_{0}$ and let $C$ be a $V$-fit Weyl chamber of $\mathcal{R}$ for $\left(\mathfrak{g}_{0}, \mathfrak{y}\right)$. Let $\mathfrak{y}=\mathfrak{q}_{\Phi}$ for a subset $\Phi$ of the set $\mathcal{B}$ of the $C$-positive simple roots. Set

$$
\Psi=\Phi \cup\{\alpha \in \mathcal{B} \mid \bar{\alpha} \prec 0\}
$$

and take $\mathfrak{q}=\mathfrak{q}_{\Psi}$. If $\alpha \succ 0$ is imaginary, then $\operatorname{supp}(\alpha)$ contains some $\beta_{0}$ with $\bar{\beta}_{0} \prec 0$. Thus, by (9.7), $\beta_{0} \in \Psi$, and therefore $\alpha \in \mathcal{Q}_{\Psi}^{n}$, showing that $\mathcal{Q}_{\Psi}^{r}$ does not contain any imaginary root. This implies that $\mathfrak{h}_{0}$ is maximally noncompact in $\mathfrak{i}_{0}$. Moreover, the equality in (9.4) is valid because of (6.9) of Proposition 6.3. Hence both (9.4) and (9.5) are satisfied.

The exactness of (9.6) follows because the $\mathbf{G}_{0}$-equivariant $C R$ map $M \rightarrow M_{\mathfrak{y}}$ is the weakly nondegenerate reduction, and hence the fibers are simply connected by Theorem 6.2, so that the argument in the proof of Theorem 9.1 applies.

In the last part of this section, we shall give an explicit description of the fundamental group $\pi_{1}(M)$ of the $\mathbf{G}_{0}$ orbit $M$.

Fix a Cartan pair $\left(\vartheta, \mathfrak{h}_{0}\right)$ adapted to the parabolic $C R$ algebra $\left(\mathfrak{g}_{0}, \mathfrak{q}\right)$ of $M$. Let $M_{\mathfrak{e}}$, with $C R$ algebra $\left(\mathfrak{g}_{0}, \mathfrak{e}\right)$, be the real core of $M$. Keeping the Cartan involution $\vartheta$ and the corresponding Cartan decomposition $\mathfrak{g}_{0}=\mathfrak{k}_{0} \oplus \mathfrak{p}_{0}$ fixed, we denote by $\mathfrak{h}_{0}^{\mathfrak{e}}$ a $\vartheta$-invariant maximally noncompact Cartan subalgebra of $\mathfrak{i}_{0}^{\mathfrak{e}}$. We choose, as we can, $\mathfrak{h}_{0}^{\mathfrak{e}}$ in such a way that $\mathfrak{h}_{0}^{\mathfrak{e}} \cap \mathfrak{k}_{0} \subset \mathfrak{h}_{0}^{+}$and $\mathfrak{h}_{0}^{\mathfrak{e}} \cap \mathfrak{p}_{0} \supset \mathfrak{h}_{0}^{-}$.

Let $\mathcal{R}^{\mathfrak{e}}$ be the root system of $\mathfrak{g}$ with respect to the complexification $\mathfrak{h}^{\mathfrak{e}}$ of $\mathfrak{h}_{0}^{\mathfrak{e}}$, and $\mathcal{E}$ the parabolic set of $\mathfrak{e}$ in $\mathcal{R}^{\mathfrak{e}}$. We recall that, for a real root $\alpha \in \mathcal{R}^{\mathfrak{e}}$, the real eigenspace:

$$
\mathfrak{g}_{0}^{\alpha}=\left\{X \in \mathfrak{g}_{0} \mid[H, X]=\alpha(H) X, \quad \forall H \in \mathfrak{h}_{0}^{\mathfrak{e}} \cap \mathfrak{p}_{0}\right\}
$$

is not trivial. Its real dimension is called the multiplicity of $\alpha$. 
Let $C \in \mathfrak{C}\left(\mathcal{R}^{\mathfrak{e}}, \mathcal{E}\right)$ be an $S$-fit Weyl chamber for $\left(\mathfrak{g}_{0}, \mathfrak{e}\right)$, denote by $\mathcal{B}$ the basis of $C$-positive simple roots in $\mathcal{R}^{\mathfrak{e}}$, and $\Phi^{\mathfrak{e}}=\mathcal{B} \cap \mathcal{E}^{n}$. The roots of $\mathcal{B}$ correspond to the nodes of a Satake diagram and, in particular, $\bar{\alpha} \succ 0$ for all non imaginary $\alpha \succ 0$.

We utilize [27] to describe the fundamental group $\pi_{1}\left(M_{\mathfrak{e}}\right)$ in terms of a set $\Gamma$ of generators, given by (9.9), and by the relations (9.10) below:

$$
\begin{aligned}
& \Gamma=\left\{\xi_{\alpha} \mid \alpha \in \mathcal{B} \cap \mathcal{R}_{\text {re }}^{\mathfrak{e}} \text { has multiplicity } 1\right\} \\
& \xi_{\alpha}=1 \quad \text { if } \alpha \notin \Phi^{\mathfrak{e}}, \quad \xi_{\alpha} \xi_{\beta}=\xi_{\beta} \xi_{\alpha}^{(-1)^{\left(\alpha \mid \beta^{\vee}\right)}} \quad \forall \xi_{\alpha}, \xi_{\beta} \in \Gamma .
\end{aligned}
$$

In (9.10) we use the standard notation $\beta^{\vee}=2 \beta /\|\beta\|^{2}$.

From Theorem 9.1 and this description of $\pi_{1}\left(M_{\mathfrak{e}}\right)$, we get:

Corollary 9.4. Let $M$ be a $\mathbf{G}_{0}$-orbit, with associated parabolic CR algebra $\left(\mathfrak{g}_{0}, \mathfrak{q}\right)$, and $M_{\mathfrak{e}}$ its real core. If $\mathfrak{g}_{0}$ is a real semisimple Lie algebra such that all its simple ideals are $^{2}$

either of the complex type, or compact, or of one of the real types

AII, AIIIa, AIV, BII, CII, DII, DIIIb, EIII, EIV, FII,

then all $\mathbf{G}_{0}$-orbits are simply connected.

If we allow the simple ideals to be either of the types listed in (a) or of the real types:

AIIIb and DIIIa,

then the map $\pi_{1}(M) \rightarrow \pi_{1}\left(M_{\mathfrak{e}}\right)$ of $(9.2)$ is an isomorphism.

Proof. Every $\mathbf{G}_{0}$-orbit splits into the Cartesian product of $\mathbf{G}_{0 i}$-orbits, each corresponding to a simple ideal $\mathfrak{g}_{0}$ of $\mathfrak{g}_{0}$ (see e.g. [2, page 490]). Thus we can reduce to the case where $\mathfrak{g}_{0}$ is simple. Consider a maximally noncompact Cartan subalgebra $\mathfrak{h}_{0}^{\mathfrak{e}}$ of $\mathfrak{g}_{0}$. Let $\mathcal{R}^{\mathfrak{e}}$ be the root system of $\mathfrak{g}$ with respect to the complexification $\mathfrak{h}^{\mathfrak{e}}$ of $\mathfrak{h}_{0}^{\mathfrak{e}}$. The first assertion follows from the fact that, in the cases listed in $(a)$, if $C$ is any $S$-fit chamber for $\left(\mathfrak{g}_{0}, \mathfrak{e}\right)$, then $\mathcal{B}$ does not contain any simple real root with multiplicity one. Hence we have $\pi_{1}\left(M_{\mathfrak{e}}\right)=1$ and thus also $M$ is simply connected.

The last statement is a consequence of the fact that in the cases listed in (b), the quotient $\mathbf{H}_{0} / \mathbf{Z}\left(\mathbf{G}_{0}\right)$ of a Cartan subgroup $\mathbf{H}_{0}$ of $\mathbf{G}_{0}$ by its center $\mathbf{Z}\left(\mathbf{G}_{0}\right)$ is always connected. Then, by the exact sequence (9.3), the fiber $F$ is connected, and therefore (9.2) yields an isomorphism of the fundamental groups of $M$ and $M_{\mathfrak{e}}$.

Remark 9.5. We note that Proposition 5.7 and Theorem 6.4 provide an effective construction of $\mathfrak{e}$, and hence of $M_{\mathfrak{e}}$, starting from a representation $\mathfrak{q}=\mathfrak{q}_{\Phi}$ in terms of the root system associated to a Cartan pair $\left(\vartheta, \mathfrak{h}_{0}\right)$ adapted to $\left(\mathfrak{g}_{0}, \mathfrak{q}\right)$.

${ }^{2}$ Here we follow, for labeling the simple real Lie algebras [14, Table VI, Chapter X]. 
Fix a Chevalley basis $\left\{X_{\alpha}\right\}_{\alpha \in \mathcal{R}^{\mathfrak{e}}} \cup\left\{H_{\alpha}\right\}_{\alpha \in \mathcal{B}}$ of $\mathfrak{g}$ (see [8]), with:

$$
X_{\alpha} \in \mathfrak{g}^{\alpha}, \quad \tau\left(X_{\alpha}\right)=X_{-\alpha}, \quad\left[H_{\alpha}, X_{ \pm \alpha}\right]= \pm 2 X_{\alpha}, \quad\left[X_{\alpha}, X_{-\alpha}\right]=-H_{\alpha} .
$$

When $\alpha \in \mathcal{R}_{\text {re }}^{\mathfrak{e}}$, we choose, as we can, $X_{\alpha} \in \mathfrak{g}_{0}$.

Let $\xi_{\alpha} \in \Gamma$. The element $\exp \left(i \pi H_{\alpha}\right)$ belongs to the normalizer of $\mathfrak{h}_{0}$ in $\mathbf{L}_{0}^{\mathfrak{e}}$, and the map in (9.1) transforms $\xi_{\alpha}$ into the equivalence class of $\exp \left(i \pi H_{\alpha}\right)$ in $\mathbf{W}\left(\mathbf{L}_{0}^{\mathfrak{e}}, \mathfrak{h}_{0}\right) / \mathbf{W}\left(\mathbf{S}_{0}^{\mathfrak{e}}, \mathfrak{h}_{0} \cap \mathfrak{s}_{0}^{\mathfrak{e}}\right)$.

The Cartan subalgebra $\mathfrak{h}_{0}$ is obtained from $\mathfrak{h}_{0}^{\mathfrak{e}}$ by the Cayley transform with respect to a set $\alpha_{1}, \ldots, \alpha_{m}$ of pairwise strongly orthogonal real roots in $\mathcal{E}^{r}$. With $\mathfrak{h}_{\mathbb{R}}^{\mathfrak{e}}=\left(\mathfrak{h}_{0}^{\mathfrak{e}} \cap \mathfrak{p}_{0}\right)+i\left(\mathfrak{h}_{0}^{\mathfrak{e}} \cap \mathfrak{k}_{0}\right)$ and $\mathfrak{h}_{\mathbb{R}}=\mathfrak{h}_{0}^{-} \oplus i \mathfrak{h}_{0}^{+}$, the Cayley transform maps $\mathfrak{h}_{\mathbb{R}}^{\mathfrak{e}}$ onto $\mathfrak{h}_{\mathbb{R}}$ by:

$$
\lambda: \mathfrak{h}_{\mathbb{R}}^{\mathfrak{e}} \ni H \longrightarrow H+\frac{i}{2} \sum_{j=1}^{m} \alpha_{j}(H)\left(i H_{\alpha_{j}}+X_{\alpha_{j}}+X_{-\alpha_{j}}\right) \in \mathfrak{h}_{\mathbb{R}} .
$$

Since $\alpha(H)=0$ for all $H \in \mathfrak{z}_{0}^{\mathfrak{e}}$ and $\alpha \in \mathcal{E}^{r}=\overline{\mathcal{E}}^{r}$, the Cayley transform is the identity on $\mathfrak{z}_{0}^{\mathfrak{e}} \subset \mathfrak{h}_{0}^{\mathfrak{e}} \cap \mathfrak{h}_{0}$.

For a real $\beta \in \Phi^{\mathfrak{e}}$, the action of $\exp \left(i \pi H_{\beta}\right)$ on $\mathfrak{h}_{\mathbb{R}}$ is described by

$$
\begin{array}{r}
\operatorname{Ad}\left(\exp \left(i \pi H_{\beta}\right)\right)\left(H+\frac{i}{2} \sum_{j=1}^{m} \alpha_{j}(H)\left(i H_{\alpha_{j}}+X_{\alpha_{j}}+X_{-\alpha_{j}}\right)\right) \\
=H+\frac{i}{2} \sum_{j=1}^{m} \alpha_{j}(H)\left(i H_{\alpha_{j}}+e^{i \pi\left(\alpha_{j} \mid \beta^{\vee}\right)}\left(X_{\alpha_{j}}+X_{-\alpha_{j}}\right)\right) .
\end{array}
$$

By duality, the Cayley transform defines a map $\lambda^{*}: \mathfrak{h}_{\mathbb{R}}^{*} \rightarrow\left(\mathfrak{h}_{\mathbb{R}}^{\mathfrak{e}}\right)^{*}$, that gives by restriction a bijection $\mathcal{R} \rightarrow \mathcal{R}^{\mathfrak{e}}$ of the two root systems. Set:

$$
\left[\lambda^{*}\right]^{-1}: \mathcal{R}^{\mathfrak{e}} \ni \alpha \longrightarrow \alpha^{\lambda} \in \mathcal{R}
$$

Then we obtain:

Lemma 9.6. Let $\beta$ be any real root in $\Phi^{\mathfrak{e}}$. Then $\operatorname{Ad}\left(\exp \left(i \pi H_{\beta}\right)\right)$ defines in the Weyl group of $\mathcal{R}$ the element:

$$
s_{\alpha_{1}^{\lambda}}^{\left(\alpha_{1} \mid \beta^{\vee}\right)} \circ \cdots \circ s_{\alpha_{m}^{\lambda}}^{\left(\alpha_{m} \mid \beta^{\vee}\right)} \in \mathbf{W}(\mathcal{R})
$$

where $s_{\alpha_{j}^{\lambda}}$ is the symmetry with respect to $\alpha_{j}^{\lambda} \in \mathcal{R}$.

Let $\mathfrak{s}_{0(j)}^{\mathfrak{e}}$, for $j=1, \ldots, p$, be the simple ideals of $\mathfrak{s}_{0}^{\mathfrak{e}}$. For each $j=1, \ldots, p$, denote by $\mathcal{R}_{(j)}$ the set of roots $\gamma \in \mathcal{R}=\mathcal{R}(\mathfrak{g}, \mathfrak{h})$ that are of the form $\gamma=\alpha^{\lambda}$ for some $\alpha \in \mathcal{R}^{\mathfrak{e}}$ for which the eigenspace $\mathfrak{g}^{\alpha}$ is contained in the complexification $\mathfrak{s}_{(j)}^{\mathfrak{e}}$ 
of $\mathfrak{s}_{0(j)}^{\mathfrak{e}}$. The $\mathcal{R}_{(j)}$ 's are disjoint. Let $\mathbf{S}_{0(j)}^{\mathfrak{e}}$ be the analytic Lie subgroup of $\mathbf{S}_{0}^{\mathfrak{e}}$ with Lie algebra $\mathfrak{s}_{0(j)}^{\mathfrak{e}}$.

For each $j=1, \ldots, p$, let $\mathcal{A}_{(j)}$ be the subset of the set $\left\{\alpha_{1}, \ldots, \alpha_{m}\right\}$ of roots of $\mathcal{R}^{\mathfrak{e}}$, used to define the Cayley transform (9.11), consisting of those for which $\alpha_{j}^{\lambda} \in \mathcal{R}_{(j)}$. Since:

$$
\begin{aligned}
\mathbf{S}_{0}^{\mathfrak{e}} & =\mathbf{S}_{0(1)}^{\mathfrak{e}} \bowtie \cdots \bowtie \mathbf{S}_{0(p)}^{\mathfrak{e}} \quad \text { and } \\
\mathbf{W}\left(\mathbf{S}_{0}^{\mathfrak{e}}, \mathfrak{h}_{0} \cap \mathfrak{s}_{0}^{\mathfrak{e}}\right) & =\mathbf{W}\left(\mathbf{S}_{0(1)}^{\mathfrak{e}}, \mathfrak{h}_{0} \cap \mathfrak{s}_{0(1)}^{\mathfrak{e}}\right) \bowtie \cdots \bowtie \mathbf{W}\left(\mathbf{S}_{0(p)}^{\mathfrak{e}}, \mathfrak{h}_{0} \cap \mathfrak{s}_{0(p)}^{\mathfrak{e}}\right)
\end{aligned}
$$

we obtain:

Theorem 9.7. With the notation above: $\pi_{1}(M)$ is the subgroup of $\pi_{1}\left(M_{\mathfrak{e}}\right)$ consisting of the elements of the form:

$$
\xi=\xi_{\beta_{1}}^{k_{1}} \cdots \xi_{\beta_{\ell}}^{k_{\ell}}
$$

where $\ell$ is a positive integer, and $k_{1}, \ldots, k_{\ell} \in \mathbb{Z}, \xi_{\beta_{1}}, \ldots, \xi_{\beta_{\ell}} \in \Gamma$ satisfy one of the two equivalent conditions:

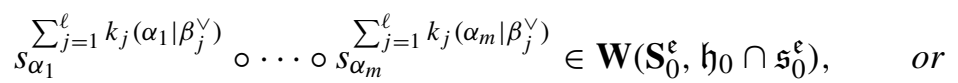

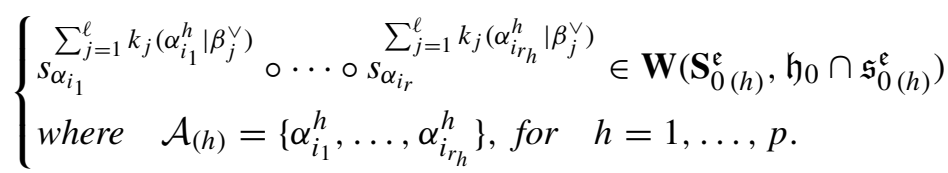

Remark 9.8. When $\mathbf{L}_{0}$ is a reductive real linear group, and $\mathfrak{h}_{0}$ a Cartan subalgebra of its Lie algebra $\mathfrak{l}_{0}$, the analytic Weyl group $\mathbf{W}\left(\mathbf{L}_{0}, \mathfrak{h}_{0}\right)$ has been explicitly computed (see e.g. $[1,26])$. Thus Theorem 9.7 yields an effective way to compute the fundamental group of a $\mathbf{G}_{0}$-orbit $M$.

For the convenience of the reader, we give below a description of that part of the Weyl group $\mathbf{W}\left(\mathbf{S}_{0}^{\mathfrak{e}}, \mathfrak{h}_{0} \cap \mathfrak{s}_{0}^{\mathfrak{e}}\right)$, that is needed to understand (9.17), (9.18). We take, as we can, $\mathbf{G}$ simply connected. Then also the complexification $\mathbf{S}^{\mathfrak{e}}$ of $\mathbf{S}_{0}^{\mathfrak{e}}$ is simply connected, because $\mathbf{S}^{\mathfrak{e}}$ is the semisimple Levi factor of a parabolic subgroup of $\mathbf{G}$. Indeed, each integral character of $\mathfrak{s}^{\mathfrak{e}}$ lifts to an integral character of $\mathfrak{g}$ and therefore defines a linear representation of $\mathbf{G}$, giving, by restriction, a linear representation of $\mathbf{S}^{\mathfrak{e}}$. It is well known that this property characterizes simple connectivity. This is the situation where we can apply the results of $[1,26]$.

Let $\mathfrak{h}$ be the complexification of $\mathfrak{h}_{0}$, and $\mathcal{S}$ the root system of $\mathfrak{s}^{\mathfrak{e}}$ with respect to the Cartan subalgebra $\mathfrak{t}=\mathfrak{h} \cap \mathfrak{s}^{\mathfrak{e}}$. Denote by $\mathcal{S}_{\text {im,c }}$ and by $\mathcal{S}_{\text {im,n }}$ the sets of compact and noncompact imaginary roots of $\mathcal{S}$, respectively. For a Weyl chamber $C \in \mathfrak{C}(\mathcal{S})$, set $\rho_{0}=\frac{1}{2} \sum\left\{\beta \in \mathcal{S}_{\text {im,c }} \mid \beta \succ 0\right\}$. Let $E$ be the subset of $\mathcal{S}_{\text {im,n }}$ of the $C$-positive noncompact imaginary roots that are orthogonal to $\rho_{0}$. The elements of $E$ are pairwise strongly orthogonal, thus the subgroup $\mathbf{W}(E)$ generated by the symmetries $s_{\alpha}$, 
for $\alpha \in E$, is isomorphic to $\mathbb{Z}_{2}^{p}$, where $p \geq 0$ is the number of elements of $E$. Let $\Lambda$ be the co-root lattice, generated by the elements $\alpha^{\vee}=2 \alpha /\|\alpha\|^{2}$ for $\alpha \in \mathcal{S}$, and $\Lambda^{*}$ the sublattice generated by the $\alpha^{\vee}$ for $\alpha \in \mathcal{S}_{\text {im }}$. Let $\mathfrak{t}_{\mathbb{R}}^{*}$ be the linear span of $\mathcal{S}$ and $\varpi$ the orthogonal projection of $\mathfrak{t}_{\mathbb{R}}^{*}$ onto the linear span of $\mathcal{S}_{\mathrm{im}}$.

Let $\mathbf{W}^{*}$ be the subgroup of the Weyl group $\mathbf{W}(\mathcal{S})$ generated by the symmetries $s_{\alpha}$ for $\alpha \in \mathcal{R}_{\mathrm{im}, \mathrm{n}}$. Then we have:

$$
\mathbf{W}^{*} \cap \mathbf{W}\left(\mathbf{S}_{0}^{\mathfrak{e}}, \mathfrak{h}_{0} \cap \mathfrak{s}_{0}^{\mathfrak{e}}\right)=\operatorname{ker}\left(f: \mathbf{W}(E) \rightarrow \Lambda^{*} / 2 \varpi(\Lambda)\right)
$$

where $f$ is the homomorphism that maps $s_{\alpha}$ to $\alpha^{\vee}$.

We note that the elements (9.16), having been constructed from real roots of the root system $\mathcal{R}^{\mathfrak{e}}$, belong to the subgroup of $\mathbf{W}(\mathcal{R})$ generated by the symmetries with respect to noncompact imaginary roots that are orthogonal to $\rho_{0}$, and therefore to $\mathbf{W}^{*}$.

\section{The Mostow fibration}

Let $M=\mathbf{G}_{0} / \mathbf{I}_{0}$ be a homogeneous space of a Lie group $\mathbf{G}_{0}$, with $\mathbf{I}_{0}$ a closed subgroup of $\mathbf{G}_{0}$. We assume that both $\mathbf{G}_{0}$ and $\mathbf{I}_{0}$ have finitely many connected components. We fix maximal compact subgroups $\mathbf{K}_{0}$ and $\mathbf{K}_{00}$ of $\mathbf{G}_{0}$ and of $\mathbf{I}_{0}$, respectively, with $\mathbf{K}_{00} \subset \mathbf{K}_{0}$. In [22] and [23] G. D. Mostow proved that there exist closed Euclidean subspaces $F$ and $E$ in $\mathbf{G}_{0}$ such that:

$$
\begin{aligned}
& \left.\begin{array}{c}
\mathbf{K}_{0} \times F \times E \ni(k, f, e) \rightarrow k \cdot f \cdot e \in \mathbf{G}_{0} \\
\text { and } \quad \mathbf{K}_{00} \times E \ni(k, e) \rightarrow k \cdot e \in \mathbf{I}_{0}
\end{array}\right\} \text { are diffeomorphisms onto, } \\
& \operatorname{ad}(k)(F)=F \quad \forall k \in \mathbf{K}_{00} .
\end{aligned}
$$

In particular, $M$ is isomorphic, as a $\mathbf{K}_{0}$-space, to the manifold $\mathbf{K}_{0} \times \mathbf{K}_{00} F$, i.e. to the quotient of the Cartesian product $\mathbf{K}_{0} \times F$ with respect to the equivalence relation that identifies $(k, f)$ and $\left(k \cdot k_{0}^{-1}, \operatorname{ad}\left(k_{0}\right)(f)\right)$ if $k \in \mathbf{K}_{0}, f \in F$, and $k_{0} \in \mathbf{K}_{00}$. Let $N$ be the homogeneous space $\mathbf{K}_{0} / \mathbf{K}_{00}$.

Definition 10.1. The $\mathbf{K}_{0}$-equivariant fibration $M \rightarrow N$ defined by the commutative diagram

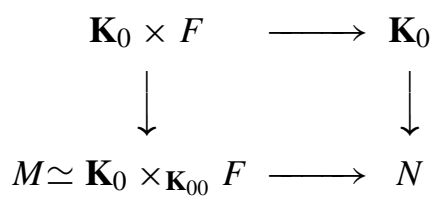

is called the Mostow fibration of $M$.

We come back now to the situation where $M$ is a $\mathbf{G}_{0}$-orbit in a complex flag manifold $X$, with associated parabolic $C R$ algebra $\left(\mathfrak{g}_{0}, \mathfrak{q}\right)$. We keep the notation of the previous sections. In particular, we denote by $\mathbf{K}$ the complexification of $\mathbf{K}_{0}$, and by $\mathfrak{k}_{0}, \mathfrak{k}$ the Lie algebras of $\mathbf{K}_{0}, \mathbf{K}$, respectively. Then the basis $N$ of the 
Mostow fibration is the intersection of $M$ with the complex $\mathbf{K}$-orbit $M^{*}$ in $X=$ $\mathbf{G} / \mathbf{Q}$, that corresponds to $M$ in the Matsuki duality (see $[9,17])$. Note that $N$ is a $\mathbf{K}_{0}$-homogeneous generic $C R$-submanifold of $M^{*}$, with associated $C R$ algebra $\left(\mathfrak{k}_{0}, \mathfrak{q} \cap \mathfrak{k}\right)$.

Proposition 10.2. The CR manifold $M$ and the basis $N$ of its Mostow fibration $M \rightarrow N$ have the same CR-codimension. In particular, the fibers of the Mostow fibration $M \rightarrow N$ have an even dimension.

Proof. The $C R$ codimensions of $M$ and $N$ are ( $c f$. Remark 1.4)

$$
\begin{aligned}
& C R-\operatorname{codim} M=\operatorname{dim}_{\mathbb{C}} \mathfrak{g}-\operatorname{dim}_{\mathbb{C}}(\mathfrak{q}+\overline{\mathfrak{q}}) \quad \text { and } \\
& C R-\operatorname{codim} N=\operatorname{dim}_{\mathbb{C}} \mathfrak{k}-\operatorname{dim}_{\mathbb{C}}([\mathfrak{q} \cap \mathfrak{k}]+[\overline{\mathfrak{q}} \cap \mathfrak{k}]) .
\end{aligned}
$$

Thus, we need to prove that

$$
\operatorname{dim}_{\mathbb{C}} \mathfrak{g}=\operatorname{dim}_{\mathbb{C}}(\mathfrak{q}+\overline{\mathfrak{q}})+\operatorname{dim}_{\mathbb{C}} \mathfrak{k}-\operatorname{dim}_{\mathbb{C}}([\mathfrak{q} \cap \mathfrak{k}]+[\overline{\mathfrak{q}} \cap \mathfrak{k}])
$$

First we show that

$$
(\mathfrak{q}+\overline{\mathfrak{q}}) \cap \mathfrak{k}=(\mathfrak{q} \cap \mathfrak{k})+(\overline{\mathfrak{q}} \cap \mathfrak{k}) .
$$

Fix any $\vartheta$-invariant Cartan subalgebra $\mathfrak{h}_{0}$ of $\mathfrak{i}_{0}$. Let $\mathfrak{h}$ be the complexification of $\mathfrak{h}_{0}$ and $\mathcal{R}=\mathcal{R}(\mathfrak{g}, \mathfrak{h})$ be the root system of $\mathfrak{g}$ with respect to its Cartan subalgebra $\mathfrak{h}$. Recall that $\mathfrak{k}=\{X+\vartheta(X) \mid X \in \mathfrak{g}\}$ and that, if $X_{\alpha} \in \mathfrak{g}^{\alpha}$, then $\vartheta\left(X_{\alpha}\right) \in \mathfrak{g}^{-\bar{\alpha}}$. To prove (10.3), it suffices to show that, if $X_{\alpha} \neq 0$ and $\left(X_{\alpha}+\vartheta\left(X_{\alpha}\right)\right) \in(\mathfrak{q}+\overline{\mathfrak{q}})$, then $\alpha$ and $-\bar{\alpha}$ both together belong either to $\mathcal{Q}$, or to $\overline{\mathcal{Q}}$. The assertion is trivially true when $\bar{\alpha}=-\alpha$. When $\bar{\alpha} \neq-\alpha$, the fact that $\left(X_{\alpha}+\vartheta\left(X_{\alpha}\right)\right) \in(\mathfrak{q}+\overline{\mathfrak{q}})$ implies that $\{\alpha,-\bar{\alpha}\} \subset \mathcal{Q} \cup \overline{\mathcal{Q}}$. If $\alpha \in \mathcal{Q} \backslash \overline{\mathcal{Q}}$ and $-\bar{\alpha} \in \overline{\mathcal{Q}} \backslash \mathcal{Q}$, then neither $\bar{\alpha}$, nor $-\bar{\alpha}$ would belong to $\mathcal{Q}$, contradicting the fact that $\mathcal{Q}$ is a parabolic set. Analogously, we rule out the case where $\alpha \in \overline{\mathcal{Q}} \backslash \mathcal{Q}$ and $-\bar{\alpha} \in \mathcal{Q} \backslash \mathcal{Q}$. This proves (10.3). Since

$$
\operatorname{dim}_{\mathbb{C}}(\mathfrak{q}+\overline{\mathfrak{q}})+\operatorname{dim}_{\mathbb{C}} \mathfrak{k}-\operatorname{dim}_{\mathbb{C}}([\mathfrak{q}+\overline{\mathfrak{q}}] \cap \mathfrak{k})=\operatorname{dim}_{\mathbb{C}}(\mathfrak{q}+\overline{\mathfrak{q}}+\mathfrak{k}),
$$

to complete the proof that $M$ and $N$ have the same $C R$ codimension it suffices to verify that:

$$
\mathfrak{q}+\overline{\mathfrak{q}}+\mathfrak{k}=\mathfrak{g}
$$

and, to this aim, that all root spaces $\mathfrak{g}^{\alpha}$ are contained in the left hand side of (10.4). If $\mathfrak{g}^{\alpha} \not \subset \mathfrak{q}+\overline{\mathfrak{q}}$, then $\alpha$ is either real or complex, and $\mathfrak{g}^{-\bar{\alpha}} \subset \mathfrak{q} \cap \overline{\mathfrak{q}}$. With $X_{\alpha} \in \mathfrak{g}^{\alpha}$, we have $\vartheta\left(X_{\alpha}\right) \in \mathfrak{g}^{-\bar{\alpha}} \subset \mathfrak{q} \cap \overline{\mathfrak{q}}$ and $\left(X_{\alpha}+\vartheta\left(X_{\alpha}\right)\right) \in \mathfrak{k}$. Thus $X_{\alpha}$ belongs to the left hand side of (10.4). This completes the proof.

Theorem 10.3. Let $M$ be a $\mathbf{G}_{0}$-orbit, with associated parabolic CR algebra $\left(\mathfrak{g}_{0}, \mathfrak{q}\right)$, and $M \rightarrow N$ its Mostow fibration. Let $M_{\mathfrak{e}}$ be the real core of $M$ (see Definition 7.1). Then there is a sequence of $\mathbf{K}_{0}$-equivariant fibrations:

$$
N=N^{(0)} \rightarrow N^{(1)} \rightarrow \cdots \rightarrow N^{(m-1)} \rightarrow N^{(m)}=M_{\mathfrak{e}},
$$

in which, for each $h \geq 1$, the fiber $L^{(h)}$ of the $\mathbf{K}_{0}$-equivariant fibration $N^{(h-1)} \rightarrow$ $N^{(h)}$ is diffeomorphic to a disjoint union of a finite number of copies of a complex flag manifold. 
Proof. We consider the sequence (7.2). For each $h=0,1, \ldots, m$ we take $N^{(h)}$ to be the basis of the Mostow fibration of the $\mathbf{G}_{0}$-orbit $M^{(h)}$. Let $\mathbf{I}_{0}^{(h)}$ be the isotropy subgroup of $M^{(h)}$ and $\mathbf{K}_{00}^{(h)}$ its maximal $\vartheta$-invariant compact subgroup. Being complex, by Theorem 5.10 each connected component of the fiber $F_{h}=F_{h}^{\prime} \times F_{h}^{\prime \prime}$ of the $\mathbf{G}_{0}$-equivariant fibration of $M_{w}^{(h-1)} \rightarrow M^{(h)}$ is $C R$-diffeomorphic to the product of a complex Euclidean space $F_{h}^{\prime \prime}$ and of the disjoint union $F_{h}^{\prime}$ of finitely many copies of an open orbit $\Omega^{(h)}$ in a complex flag manifold $X_{h}$. Then $L^{(h)}$ is the basis of the Mostow fibration $F_{h}^{\prime} \rightarrow L^{(h)}$. Thus $L^{(h)}$ has finitely many connected components, each diffeomorphic to a complex flag manifold, being equal to the Matsuki-dual of an open orbit.

Thus we have:

Corollary 10.4. With the notation of Theorem 10.3:

$$
\chi(N)=0 \Longleftrightarrow \chi\left(M_{\mathfrak{e}}\right)=0
$$

where $\chi$ is the Euler-Poincaré characteristic.

Let $\left\{M_{j} \mid j \in J\right\}$ be the set of all $\mathbf{G}_{0}$-orbits in the complex flag manifold $X=$ G/Q. Then:

$$
\chi(X)=\sum_{j \in J} \chi\left(N_{j}\right)
$$

where $N_{j}$ is the basis of the Mostow fibration $M_{j} \rightarrow N_{j}$.

Proof. We keep the notation of Theorem 10.3. The Euler-Poincare characteristic $\chi(N)$ is the product $\chi(N)=\chi\left(M_{\mathfrak{e}}\right) \cdot \chi\left(L^{(1)}\right) \cdots \chi\left(L^{(m)}\right)$ and $\chi\left(L^{(h)}\right)>0$ for $h=1, \ldots, m$, because each $L^{(h)}$ is a complex flag manifold.

The last assertion follows by considering a cell decomposition $\mathcal{C}$ of $X$ in which every cell is contained in some $\mathbf{G}_{0}$-orbit $M_{j}$. Since the fibers of the Mostow fibration are Euclidean, we may obtain a cell decomposition of $M_{j}$ from a cell decomposition of the basis $N_{j}$ of its Mostow fibration. Since the dimension of the fibers is even, the contribution of the cells of $M_{j}$ to the alternated sum $\chi(X)=$ $\sum_{c \in \mathcal{C}}(-1)^{\operatorname{dim}(c)}$ of the cells contained in $\chi(X)$ is exactly $\chi\left(N_{j}\right)$.

\section{Real core and algebraic arc components}

In this section we compare our construction of Section 7 with the algebraic arc components of [28, Section 8]. They were defined in the following way. 
Definition 11.1. Let $M$ be a $\mathbf{G}_{0}$-orbit, with associated parabolic $C R$ algebra $\left(\mathfrak{g}_{0}, \mathfrak{q}\right)$. Let $\left(\vartheta, \mathfrak{h}_{0}\right)$ be an adapted Cartan pair and $\mathcal{R}=\mathcal{R}(\mathfrak{g}, \mathfrak{h})$. Define

$$
\begin{aligned}
\delta & =\sum_{\alpha \in \mathcal{Q}^{n} \cap \overline{\mathcal{Q}}^{n}} \alpha, \\
\mathcal{Q}_{a} & =\{\alpha \in \mathcal{R} \mid(\delta \mid \alpha) \geq 0\}, \\
\mathfrak{q}_{a} & =\mathfrak{h} \oplus \sum_{\alpha \in \mathcal{Q}_{a}} \mathfrak{g}^{\alpha} .
\end{aligned}
$$

The $\mathbf{G}_{0}$-orbit $M_{a}$, corresponding to the parabolic $C R$ algebra $\left(\mathfrak{g}_{0}, \mathfrak{q}_{a}\right)$, is called the space of algebraic arc components of $M$.

Lemma 11.2. If $\left(\mathfrak{g}_{0}, \mathfrak{q}\right)$ and $\left(\mathfrak{g}_{0}, \mathfrak{q}^{\prime}\right)$ are parabolic CR algebras with $\mathfrak{q}+\overline{\mathfrak{q}}=\mathfrak{q}^{\prime}+\overline{\mathfrak{q}}^{\prime}$, then $\mathfrak{q}_{a}=\mathfrak{q}_{a}^{\prime}$. This holds in particular when $\left(\mathfrak{g}_{0}, \mathfrak{q}^{\prime}\right)$ is the weakly nondegenerate reduction of $\left(\mathfrak{g}_{0}, \mathfrak{q}\right)$.

Proof. Indeed $\mathcal{Q}^{n} \cap \overline{\mathcal{Q}}^{n}=\mathcal{R} \backslash(-\mathcal{Q} \cup-\overline{\mathcal{Q}})=\mathcal{R} \backslash\left(-\mathcal{Q}^{\prime} \cup-\overline{\mathcal{Q}}^{\prime}\right)=\mathcal{Q}^{\prime n} \cap \overline{\mathcal{Q}}^{\prime n}$.

Lemma 11.3. Let $\left(\mathfrak{g}_{0}, \mathfrak{q}\right)$ be a parabolic CR algebra. Fix an adapted Cartan pair $\left(\vartheta, \mathfrak{h}_{0}\right)$. Let $\mathcal{R}$ be the root system of $\mathfrak{g}$ with respect to the complexification $\mathfrak{h}$ of $\mathfrak{h}_{0}$ and $\mathcal{Q} \subset \mathcal{R}$ the parabolic set of $\mathfrak{q}$. Consider the complement of $\mathfrak{q} \cap \overline{\mathfrak{q}}$ in $\mathfrak{q}$ given by

$$
\mathfrak{x}=\sum_{\alpha \in \mathcal{Q} \backslash \overline{\mathcal{Q}}} \mathfrak{g}^{\alpha} .
$$

If $\left(\mathfrak{g}_{0}, \mathfrak{q}\right)$ is weakly nondegenerate, then

$$
\{Z \in \mathfrak{x} \mid[Z, \overline{\mathfrak{q}}] \subset(\mathfrak{q}+\overline{\mathfrak{q}})\} \subset \mathfrak{q}^{n} .
$$

Proof. Set $\mathcal{Q}^{-n}=\mathcal{R} \backslash \mathcal{Q}=\left\{-\alpha \mid \alpha \in \mathcal{Q}^{n}\right\}$ and $\overline{\mathcal{Q}}^{-n}=\left\{\bar{\alpha} \mid \alpha \in \mathcal{Q}^{-n}\right\}$. We apply an argument similar to that of the proof of [2, Proposition 12.3]. The left hand side of (11.5) decomposes into a direct sum of root spaces $\mathfrak{g}^{\alpha}$. Since

$$
(\mathcal{Q} \backslash \overline{\mathcal{Q}})=\left(\mathcal{Q}^{r} \cap \overline{\mathcal{Q}}^{-n}\right) \cup\left(\mathcal{Q}^{n} \cap \overline{\mathcal{Q}}^{-n}\right),
$$

it suffices to show that the left hand side of (11.5) does not contain $\mathfrak{g}^{\alpha}$ if $\alpha \in \mathcal{Q}^{r} \cap$ $\overline{\mathcal{Q}}^{-n}$. By the assumption that $\left(\mathfrak{g}_{0}, \mathfrak{g}\right)$ is weakly nondegenerate, by $[21$, Theorem 6.2] there exist roots $\beta_{1}, \ldots, \beta_{k} \in \overline{\mathcal{Q}}$ such that:

(i) $\gamma_{i}=\alpha+\sum_{j=1}^{i} \beta_{i} \in \mathcal{R}$ for $j=1, \ldots, k$,

(ii) $\gamma_{k} \notin \mathcal{Q} \cup \overline{\mathcal{Q}}$.

If we take a sequence $\left(\beta_{1}, \ldots, \beta_{k}\right)$ satisfying (i) and (ii) with $k$ minimal, then (i) and (ii) hold true for all sequences $\left(\beta_{s_{1}}, \ldots, \beta_{s_{k}}\right)$ obtained by a permutation of the $\beta_{i}$ 's. At least one of the $\beta_{i}$ 's does not belong to $\mathcal{Q}$, so we can assume that $\beta_{1} \in \mathcal{Q}^{-n}$. Then $\gamma_{1} \in \mathcal{Q}^{-n}$, because $\alpha \in \mathcal{Q}^{r}$, and $\mathcal{Q}^{r} \cup \mathcal{Q}^{-n}$ is a parabolic set. We also have $\gamma_{1} \in \overline{\mathcal{Q}}^{-n}$, because $\beta_{2}, \ldots, \beta_{k} \in \overline{\mathcal{Q}}$ and $\gamma_{k}=\gamma_{1}+\sum_{j=2}^{k} \beta_{k} \in \overline{\mathcal{Q}}^{-n}$. This shows that $k=1$ and hence $\mathfrak{g}^{\alpha}$ is not contained in the left hand side of (11.5). 
Lemma 11.4. If $\left(\mathfrak{g}_{0}, \mathfrak{q}\right)$ is weakly nondegenerate, then

$$
\mathfrak{q}_{w} \cap \overline{\mathfrak{q}}_{w} \subset \mathfrak{q}_{a} \subset \mathfrak{q}_{w}+\overline{\mathfrak{q}}_{w} .
$$

Proof. We keep the notation introduced in the proof of the previous lemma.

Let us consider the normalizer of $\mathfrak{q}^{n} \cap \overline{\mathfrak{q}}^{n}$ in $\mathfrak{g}$ :

$$
\mathbf{N}_{\mathfrak{g}}\left(\mathfrak{q}^{n} \cap \overline{\mathfrak{q}}^{n}\right)=\left\{Z \in \mathfrak{g} \mid\left[Z, \mathfrak{q}^{n} \cap \overline{\mathfrak{q}}^{n}\right] \subset \mathfrak{q}^{n} \cap \overline{\mathfrak{q}}^{n}\right\} .
$$

The first inclusion in (11.6) is obvious, because $\mathfrak{q}_{w} \cap \overline{\mathfrak{q}}_{w}=\mathfrak{q} \cap \overline{\mathfrak{q}}$, and $\mathfrak{q}_{a}$ contains $\mathbf{N}_{\mathfrak{g}}\left(\mathfrak{q}^{n} \cap \overline{\mathfrak{q}}^{n}\right) \supset \mathfrak{q} \cap \overline{\mathfrak{q}}$. Since $\mathbf{N}_{\mathfrak{g}}\left(\mathfrak{q}^{n} \cap \overline{\mathfrak{q}}^{n}\right)$ is the direct sum of $\mathfrak{h}$ and of root spaces, we can decompose $\mathbf{N}_{\mathfrak{g}}\left(\mathfrak{q}^{n} \cap \overline{\mathfrak{q}}^{n}\right)$ into the direct sum of its nilradical $\mathbf{N}_{\mathfrak{g}}^{n}\left(\mathfrak{q}^{n} \cap \overline{\mathfrak{q}}^{n}\right)$ and of a $\tau$-invariant reductive complement $\mathbf{N}_{\mathfrak{g}}^{r}\left(\mathfrak{q}^{n} \cap \overline{\mathfrak{q}}^{n}\right.$ ) (recall that $\tau \stackrel{\mathfrak{g}}{=} \sigma \circ \vartheta$ is the conjugation with respect to the compact form $\mathfrak{u}_{0}=\mathfrak{k}_{0}+i \mathfrak{p}_{0}$ of $\mathfrak{g}$ ). Wolf proved (see [28, Theorem 8.5(2)]) that

$$
\mathbf{N}_{\mathfrak{g}}^{n}\left(\mathfrak{q}^{n} \cap \overline{\mathfrak{q}}^{n}\right) \subset \mathfrak{q}_{a}^{n}, \quad \mathbf{N}_{\mathfrak{g}}^{r}\left(\mathfrak{q}^{n} \cap \overline{\mathfrak{q}}^{n}\right) \subset \mathfrak{q}_{a}^{r},
$$

so that in particular

$$
\mathbf{N}_{\mathfrak{g}}^{n}\left(\mathfrak{q}^{n} \cap \overline{\mathfrak{q}}^{n}\right)=\mathbf{N}_{\mathfrak{g}}\left(\mathfrak{q}^{n} \cap \overline{\mathfrak{q}}^{n}\right) \cap \mathfrak{q}_{a}^{n}, \quad \mathbf{N}_{\mathfrak{g}}^{r}\left(\mathfrak{q}^{n} \cap \overline{\mathfrak{q}}^{n}\right)=\mathbf{N}_{\mathfrak{g}}\left(\mathfrak{q}^{n} \cap \overline{\mathfrak{q}}^{n}\right) \cap \mathfrak{q}_{a}^{r} .
$$

To complete the proof of (11.6), it suffices to show that $\mathcal{Q}_{a} \subset\left(\mathcal{Q}_{w} \cup \overline{\mathcal{Q}}_{w}\right)$. Since (see [28, Theorem 8.5(2)]) $\mathfrak{q}_{a}$ is contained in $\mathfrak{q}+\overline{\mathfrak{q}}$ and is invariant by conjugation, we can assume, by substituting, if needed, $\bar{\alpha}$ to $\alpha$, that $\alpha \in \mathcal{Q}$. Assume by contradiction that $\alpha \notin \mathcal{Q}_{w} \cup \overline{\mathcal{Q}}_{w}$. Since we have the partition

$$
\mathcal{R} \backslash\left(\mathcal{Q}_{w} \cup \overline{\mathcal{Q}}_{w}\right)=\left(\mathcal{Q}^{r} \cap \overline{\mathcal{Q}}^{-n}\right) \cup\left(\overline{\mathcal{Q}}^{r} \cap \mathcal{Q}^{-n}\right) \cup\left(\mathcal{Q}^{-n} \cap \overline{\mathcal{Q}}^{-n}\right),
$$

we obtain that $\alpha \in \mathcal{Q} \backslash\left(\mathcal{Q}_{w} \cup \overline{\mathcal{Q}}_{w}\right)=\mathcal{Q}^{r} \cap \overline{\mathcal{Q}}^{-n}$. This implies that $\mathfrak{g}^{-\alpha}$ is contained in $\mathbf{N}_{\mathfrak{g}}\left(\mathfrak{q}^{n} \cap \overline{\mathfrak{q}}^{n}\right) \subset \mathfrak{q}_{a}$. Then $\mathfrak{g}^{ \pm \alpha} \subset \mathfrak{q}_{a}^{r}$, and hence, by (11.9), $\mathfrak{g}^{ \pm \alpha} \subset \mathbf{N}_{\mathfrak{g}}^{r}\left(\mathfrak{q}^{n} \cap \overline{\mathfrak{q}}^{n}\right)$. But $\mathbf{N}_{\mathfrak{g}}^{r}\left(\mathfrak{q}^{n} \cap \overline{\mathfrak{q}}^{n}\right)$ also normalizes $\left(\mathfrak{q}^{-n} \cap \overline{\mathfrak{q}}^{-n}\right)$, and hence $(\mathfrak{q}+\overline{\mathfrak{q}})$. By Lemma 11.3 this implies that $\alpha \in \mathcal{Q}^{n} \subset \mathcal{Q}_{w}$, yielding a contradiction.

Example 11.5. Denote by $\mathcal{F}_{d_{1}, d_{2}, \ldots, d_{r}}^{7}$ the complex manifold consisting of the flags $\left(\ell_{1}, \ell_{2}, \ldots, \ell_{r}\right)$ where $\ell_{j}$ is a $d_{j}$-dimensional linear complex subspace of $\mathbb{C}^{7}$ and $\ell_{1} \subsetneq \ell_{2} \subsetneq \cdots \subsetneq \ell_{r}$.

Let $\left(\mathrm{e}_{1}, \ldots, \mathrm{e}_{7}\right)$ be a basis of the real form $\mathbb{R}^{7}$ of $\mathbb{C}^{7}$, and $\left(\epsilon_{1}, \ldots, \epsilon_{7}\right)$ the basis of $\mathbb{C}^{7}$ given by

$$
\begin{gathered}
\epsilon_{1}=\mathrm{e}_{1}+i \mathrm{e}_{7}, \quad \epsilon_{2}=\mathrm{e}_{2}, \quad \epsilon_{3}=\mathrm{e}_{3}+i \mathrm{e}_{6}, \quad \epsilon_{4}=\mathrm{e}_{4}, \\
\epsilon_{5}=\mathrm{e}_{5}, \quad \epsilon_{6}=\mathrm{e}_{3}-i \mathrm{e}_{6}, \quad \epsilon_{7}=\mathrm{e}_{1}-i \mathrm{e}_{7} .
\end{gathered}
$$

Let $\mathbf{Q}$ be the Borel subgroup of $\mathbf{G}=\mathbf{S L}(7, \mathbb{C})$ that stabilizes the complete flag

$$
\varepsilon=\left(\left\langle\epsilon_{1}\right\rangle,\left\langle\epsilon_{1}, \epsilon_{2}\right\rangle, \ldots,\left\langle\epsilon_{1}, \ldots, \epsilon_{6}\right\rangle\right) \in \mathcal{F}_{1,2,3,4,5,6}^{7} .
$$


Set $\mathbf{G}_{0}=\mathbf{S L}(7, \mathbb{R})$, and consider the orbit $M=\mathbf{G}_{0} \cdot \varepsilon \subset \mathcal{F}_{1,2,3,4,5,6}^{7}$, with associated $C R$ algebra $\left(\mathfrak{g}_{0}, \mathfrak{q}\right)$, where $\mathfrak{g}_{0}=\mathfrak{s l}(7, \mathbb{R})$ and $\mathfrak{q}$ is the Lie algebra of $\mathbf{Q}$. The weakly nondegenerate reduction of $M$ is the $\mathbf{G}_{0}$-orbit $M^{(0)} \subset \mathcal{F}_{2,4}^{7}$ through the flag

$$
\varepsilon^{(0)}=\left(\left\langle\epsilon_{1}, \epsilon_{2}\right\rangle,\left\langle\epsilon_{1}, \epsilon_{2}, \epsilon_{3}, \epsilon_{4}\right\rangle\right) \in \mathcal{F}_{2,4}^{7}
$$

Continuing with the construction of Section 7 we obtain that $M^{(1)} \subset \mathcal{F}_{1,3,5,6}^{7}$ is the $\mathbf{G}_{0}$-orbit through the flag

$$
\varepsilon^{(1)}=\left(\left\langle\epsilon_{2}\right\rangle,\left\langle\epsilon_{2}, \epsilon_{1}, \epsilon_{4}\right\rangle,\left\langle\epsilon_{2}, \epsilon_{1}, \epsilon_{4}, \epsilon_{3}, \epsilon_{7}\right\rangle,\left\langle\epsilon_{2}, \epsilon_{1}, \epsilon_{4}, \epsilon_{3}, \epsilon_{7}, \epsilon_{6}\right\rangle\right) \in \mathcal{F}_{1,3,5,6}^{7},
$$

and $M^{(2)} \subset \mathcal{F}_{1,2,4,6}^{7}$ is the $\mathbf{G}_{0}$-orbit through the flag

$$
\varepsilon^{(2)}=\left(\left\langle\epsilon_{2}\right\rangle,\left\langle\epsilon_{2}, \epsilon_{4}\right\rangle,\left\langle\epsilon_{2}, \epsilon_{4}, \epsilon_{1}, \epsilon_{7}\right\rangle,\left\langle\epsilon_{2}, \epsilon_{4}, \epsilon_{1}, \epsilon_{7}, \epsilon_{3}, \epsilon_{6}\right\rangle\right) \in \mathcal{F}_{1,2,4,6}^{7} .
$$

Being totally real, $M^{(2)}$ coincides with $M_{\mathfrak{e}}$.

Denote by $\lambda^{*}: \mathcal{R} \rightarrow \mathcal{R}^{\mathfrak{e}}$ the Cayley transform with respect to the roots $\epsilon_{1}-\epsilon_{7}$ and $\epsilon_{3}-\epsilon_{6}$. According to (9.9), (9.10), the fundamental group of $M_{\mathfrak{e}}$ is generated by $\xi_{1}=\xi_{\lambda^{*}\left(\epsilon_{2}-\epsilon_{4}\right)}, \xi_{2}=\xi_{\lambda^{*}}\left(\epsilon_{4}-\epsilon_{1}\right), \xi_{4}=\xi_{\lambda^{*}\left(\epsilon_{7}-\epsilon_{3}\right)}$ and $\xi_{6}=\xi_{\lambda^{*}\left(\epsilon_{6}-\epsilon_{5}\right)}$, with relations $\xi_{i}^{2}=1$ and $\xi_{i} \xi_{j}=\xi_{j} \xi_{i}$ for $i, j=1,2,4,6$.

The semisimple part $\mathfrak{s}_{0}^{\mathfrak{e}}$ of the isotropy subgroup of $M_{\mathfrak{e}}$ is the subgroup corresponding to the root subsystem $\left\{ \pm\left(\epsilon_{1}-\epsilon_{7}\right), \pm\left(\epsilon_{3}-\epsilon_{6}\right)\right\}$, the subset $E$ in Remark 9.8 is $\left\{\epsilon_{1}-\epsilon_{7}, \epsilon_{3}-\epsilon_{6}\right\}$ and the map $f: \mathbf{W}(E) \rightarrow \Lambda^{*} / 2 \varpi(\Lambda)$ is a bijection. By Theorem 9.7 the fundamental group of $M$ is generated by $\xi_{1}$ and $\xi_{2} \xi_{4} \xi_{6}$ and is isomorphic to $\mathbb{Z}_{2}^{2}$.

The space of algebraic arc components of $M$ is the $\mathbf{G}_{0}$-orbit $M_{a} \subset \mathcal{F}_{1,4,6}^{7}$ through the flag

$$
\varepsilon^{(a)}=\left(\left\langle\epsilon_{2}\right\rangle,\left\langle\epsilon_{2}, \epsilon_{4}, \epsilon_{1}, \epsilon_{7}\right\rangle,\left\langle\epsilon_{2}, \epsilon_{4}, \epsilon_{1}, \epsilon_{7}, \epsilon_{3}, \epsilon_{6}\right\rangle\right) \in \mathcal{F}_{1,4,6}^{7},
$$

showing that in this case $\mathfrak{e} \subsetneq \mathfrak{q}_{a}$. Furthermore, the algebraic arc components are not simply connected, and their fundamental group is isomorphic to $\mathbb{Z}_{2}$.

Example 11.6. We keep the notation of the previous example. We consider now the basis $\left(w_{1}, \ldots, w_{7}\right)$ of $\mathbb{C}^{7}$ given by

$$
\begin{gathered}
w_{1}=e_{1}+i e_{7}, \quad w_{2}=e_{2}, \quad w_{3}=e_{3}, \quad w_{4}=e_{4} \\
w_{5}=e_{5}, \quad w_{6}=e_{6}, \quad w_{7}=e_{1}-i e_{7} .
\end{gathered}
$$

With $\mathbf{G}_{0}=\mathbf{S L}(7, \mathbb{R})$, we consider the $\mathbf{G}_{0}$-orbit $M \subset \mathcal{F}_{2,3}^{7}$ through the flag

$$
\omega=\left(\left\langle w_{1}, w_{2}\right\rangle,\left\langle w_{1}, w_{2}, w_{3}\right\rangle\right) \in \mathcal{F}_{2,3}^{7} .
$$

Then $M_{\mathfrak{e}}=M^{(1)} \subset \mathcal{F}_{1,4}^{7}$ is the $\mathbf{G}_{0}$-orbit through the flag

$$
\omega^{(1)}=\left(\left\langle w_{2}\right\rangle,\left\langle w_{1}, w_{2}, w_{3}, w_{7}\right\rangle\right) \in \mathcal{F}_{1,4}^{7} .
$$


On the other hand, the space $M_{a}$ of the algebraic arc components of $M$ is the $\mathbf{G}_{0^{-}}$ orbit $M_{a} \subset \mathcal{F}_{1,2,4}^{7}$ through the flag

$$
\omega^{(a)}=\left(\left\langle w_{2}\right\rangle,\left\langle w_{2}, w_{3}\right\rangle,\left\langle w_{1}, w_{2}, w_{3}, w_{7}\right\rangle\right) \in \mathcal{F}_{1,2,4}^{7}
$$

Thus we obtain in this case that $\mathfrak{q}_{a} \subsetneq \mathfrak{e}$.

Proposition 11.7. Let $\left(\mathfrak{g}_{0}, \mathfrak{q}\right)$ be a parabolic CR algebra, $\left(\vartheta, \mathfrak{h}_{0}\right)$ an adapted Cartan pair, $\mathcal{R}$ the root system of $\mathfrak{g}$ with respect to the complexification of $\mathfrak{h}_{0}$, and $\mathcal{Q}$ the parabolic set of $\mathfrak{q}$. Assume that there is an $S$-fit Weyl chamber in $\mathfrak{C}(\mathcal{R}, \mathcal{Q})$ with

$$
\bar{\alpha} \succ 0 \quad \text { if } \quad \alpha \in \mathcal{R}_{\mathrm{cpx}} \quad \text { and } \quad \alpha \succ 0
$$

Then:

$$
\begin{aligned}
& \mathfrak{q}_{a}=\mathfrak{e} \\
& \mathfrak{q}_{w}+\overline{\mathfrak{q}}_{w} \text { is a Lie subalgebra of } \mathfrak{g}, \\
& \text { if moreover }\left(\mathfrak{g}_{0}, \mathfrak{q}\right) \text { is weakly nondegenerate, then } \mathfrak{e}=\mathfrak{q}_{w}+\overline{\mathfrak{q}}_{w} .
\end{aligned}
$$

In particular $(11.11)$ holds true when $\left(\mathfrak{g}_{0}, \mathfrak{q}\right)$ is the parabolic CR algebra corresponding to a closed $\mathbf{G}_{0}$-orbit.

Proof. Fix an adapted Cartan pair $\left(\vartheta, \mathfrak{h}_{0}\right)$ and choose an $S$-fit Weyl chamber $C$ for $\left(\mathfrak{g}_{0}, \mathfrak{q}\right)$, such that $(11.10)$ is satisfied. We have $\mathfrak{q}_{w}+\overline{\mathfrak{q}}_{w}=\mathfrak{q}^{n}+\overline{\mathfrak{q}}^{n}+(\mathfrak{q} \cap \overline{\mathfrak{q}})$. Hence, to prove (11.12), we only need to show that, if a root $\gamma$ is the sum $\gamma=\alpha+\beta$ of a root $\alpha \in \mathcal{Q}^{n}$ and a root $\beta \in \overline{\mathcal{Q}}^{n} \backslash \mathcal{Q}$, then $\gamma \in \mathcal{Q}_{w} \cup \overline{\mathcal{Q}}_{w}$. Because of (11.10), $\beta$ is a negative imaginary root. If also $\alpha$ is imaginary, then $\gamma$ is imaginary and hence belongs to $\mathcal{Q}_{w} \cup \overline{\mathcal{Q}}_{w}$. If $\alpha$ is not imaginary, then $\gamma$ is positive and not imaginary, and thus belongs to $\mathcal{Q} \cap \overline{\mathcal{Q}}$.

Statement (11.13) follows from (11.12).

While proving (11.11), by Lemma 11.2 we can assume that $\left(\mathfrak{g}_{0}, \mathfrak{q}\right)=\left(\mathfrak{g}_{0}, \mathfrak{q}^{(0)}\right)$ is weakly nondegenerate. Since by (11.13) we have in this case $\mathfrak{q}_{w}+\overline{\mathfrak{q}}_{w}=\mathfrak{q}^{(1)}=\mathfrak{e}$, the inclusion $\mathfrak{q}_{a} \subset \mathfrak{e}$ follows by Lemma 11.4 .

We claim that $\mathfrak{e} \subset \mathbf{N}_{\mathfrak{g}}\left(\mathfrak{q}^{n} \cap \overline{\mathfrak{q}}^{n}\right)+\sum_{\alpha \in \mathcal{R}_{\text {im }}} \mathfrak{g}^{\alpha}$. Indeed let $\alpha \in \mathcal{R}$ be a root for which $\mathfrak{g}^{\alpha} \subset \mathfrak{e}$ but $\mathfrak{g}^{\alpha} \not \subset \mathbf{N}_{\mathfrak{g}}\left(\mathfrak{q}^{n} \cap \overline{\mathfrak{q}}^{n}\right)$. Since $\mathfrak{e}=\mathfrak{q}^{n}+\overline{\mathfrak{q}}^{n}+(\mathfrak{q} \cap \overline{\mathfrak{q}})$, then either $\alpha \in \mathcal{Q}^{n} \backslash \overline{\mathcal{Q}}$, or $\alpha \in \overline{\mathcal{Q}}^{n} \backslash \mathcal{Q}$. By (11.10), in both cases we have $\alpha \in \mathcal{R}_{\text {im. }}$. Since $\mathbf{N}_{\mathfrak{g}}\left(\mathfrak{q}^{n} \cap \overline{\mathfrak{q}}^{n}\right) \subset \mathfrak{q}_{a}$ by [28, Theorem 8.5(2)], and $\sum_{\alpha \in \mathcal{R}_{\mathrm{im}}} \mathfrak{g}^{\alpha} \subset \mathfrak{q}_{a}$ because $\mathfrak{q}_{a}$ is the complexification of a real parabolic subalgebra of $\mathfrak{g}_{0}$, we obtain that $\mathfrak{e} \subset \mathfrak{q}_{a}$.

The last statement is a consequence of the fact (see e.g. [3]) that for a closed orbit there are $S$-fit Weyl chambers for which (11.10) holds true. 


\section{References}

[1] J. ADAMS and F. DU CLOUX, Algorithims for representation theory of real groups, J. Inst. Math. Jussieu 8 (2009), 209-256.

[2] A. Altomani, C. Medori and M. NaCinovich, The CR structure of minimal orbits in complex flag manifolds, J. Lie Theory 16 (2006), 483-530.

[3] A. Altomani, C. Medori and M. Nacinovich, On the topology of minimal orbits in complex flag manifolds, Tohoku Math. J. 60 (2008), 403-422.

[4] S. ARAKI, On root systems and an infinitesimal classification of irreducible symmetric spaces, J. Math. Osaka City Univ. 13 (1962), 1-34.

[5] M. S. Baouendi, P. Ebenfelt and L. P. Rothschild, "Real Submanifolds in Complex Space and their Mappings", Vol. 47, Princeton University Press, Princeton, NJ, 1999.

[6] J. Berndt, S. CONSOlE and A. Fino, On index number and topology of flag manifolds, Differential Geom. Appl. 15 (2001), 81-90.

[7] T. BLOOM and I. GRAHAM, A geometric characterization of points of type $m$ on real submanifolds of $\mathbf{C}^{n}$, J. Differential Geom. 12 (1977), 171-182.

[8] N. BourbaKI, "Éléments de mathématique", Hermann, Paris, 1975, Fasc. XXXVIII: Groupes et algèbres de Lie. Chapitre VII: Sous-algèbres de Cartan, éléments réguliers. Chapitre VIII: Algèbres de Lie semi-simples déployées, Actualités Scientifiques et Industrielles, No. 1364.

[9] R. BREMigAn and J. LoRCH, Orbit duality for flag manifolds, Manuscripta Math. 109 (2002), 233-261.

[10] L. Casian and R. J. Stanton, Schubert cells and representation theory, Invent. Math. 137 (1999), 461-539.

[11] G. FELS, Locally homogeneous finitely nondegenerate CR-manifolds, Math. Res. Lett. 14 (2007), 893-922.

[12] G. Fels, A. T. Huckleberry and J. A. Wolf, "Cycle Spaces of Flag Domains. A Complex Geometric Viewpoint”, Progress in Mathematics, Vol. 245, Birkhäuser Boston Inc., Boston, MA, 2006.

[13] A. FINO, Systems of roots and topology of complex flag manifolds, Geom. Dedicata 71 (1998), 299-308.

[14] S. Helgason, "Differential Geometry, Lie Groups, and Symmetric Spaces", Pure and Applied Mathematics, Vol. 80, Academic Press, New York, 1978.

[15] J. E. HumPhrEYS, "Linear Algebraic Groups", n. 21, Springer-Verlag, New York Graduate Texts in Mathematics, 1975.

[16] A. W. KNAPP, "Lie Groups Beyond an Introduction", second ed., Progress in Mathematics, Vol. 140, Birkhäuser Boston Inc., Boston, MA, 2002.

[17] T. MATSUKI, Closure relations for orbits on affine symmetric spaces under the action of parabolic subgroups. Intersections of associated orbits, Hiroshima Math. J. 18 (1988), $59-67$.

[18] C. Medori and M. NACINOVICH, Levi-Tanaka algebras and homogeneous CR manifolds, Compositio Math. 109 (1997), 195-250.

[19] C. Medori and M. NAcinovich, Classification of semisimple Levi-Tanaka algebras, Ann. Mat. Pura Appl. 174 (1998), 285-349.

[20] C. MEdORI and M. NACINOviCH, Complete nondegenerate locally standard CR manifolds, Math. Ann. 317 (2000), 509-526.

[21] C. MEDORI and M. NACINOVICH, Algebras of infinitesimal CR automorphisms, J. Algebra 287 (2005), 234-274.

[22] G. D. Mostow, On covariant fiberings of Klein spaces, Amer. J. Math. 77 (1955), 247278.

[23] G. D. Mostow, Covariant fiberings of Klein spaces. II, Amer. J. Math. 84 (1962), 466474.

[24] M. TAKeUCHI, Two-number of symmetric R-spaces, Nagoya Math. J. 115 (1989), 43-46. 
[25] M. TAKeUCHI and S. KobAYAShi, Minimal imbeddings of $R$-spaces, J. Differential Geom. 2 (1968), 203-215.

[26] D. A. Vogan Jr., Irreducible characters of semisimple Lie groups. IV. Charactermultiplicity duality, Duke Math. J. 49 (1982), 943-1073.

[27] M. Wiggerman, The fundamental group of a real flag manifold, Indag. Mat. 9 (1998), $141-153$.

[28] J. A. WOLF, The action of a real semisimple group on a complex flag manifold. I. Orbit structure and holomorphic arc components, Bull. Amer. Math. Soc. 75 (1969), 1121-1237.

University of Luxembourg

162a, avenue de la Faïencerie

L-2309 Luxembourg

andrea.altomani@uni.lu

Dipartimento di Matematica

Università di Parma

Viale G. P. Usberti, 53/A

43100 Parma, Italia

costantino.medori@unipr.it

Dipartimento di Matematica

II Università di Roma "Tor Vergata"

Via della Ricerca Scientifica

00133 Roma, Italia

nacinovi@mat.uniroma2.it 